Portland State University

PDXScholar

Fall 11-6-2014

\title{
Mimicking Metabolism of a Reversed Chloroquine Antimalarial
}

Kelsie Lynn Kendrick

Portland State University

Follow this and additional works at: https://pdxscholar.library.pdx.edu/open_access_etds

Part of the Medicinal-Pharmaceutical Chemistry Commons Let us know how access to this document benefits you.

Recommended Citation

Kendrick, Kelsie Lynn, "Mimicking Metabolism of a Reversed Chloroquine Antimalarial" (2014).

Dissertations and Theses. Paper 2085.

https://doi.org/10.15760/etd.2083

This Thesis is brought to you for free and open access. It has been accepted for inclusion in Dissertations and Theses by an authorized administrator of PDXScholar. Please contact us if we can make this document more accessible: pdxscholar@pdx.edu. 
Mimicking Metabolism of a Reversed Chloroquine Antimalarial

by

Kelsie Lynn Kendrick

A thesis submitted in partial fulfillment of the requirements for the degree of

Master of Science

in

Chemistry

Thesis Committee:

David Peyton, Chair

David Stuart

Robert Strongin

Portland State University

2014 
Copyright (C) 2014 Kelsie Lynn Kendrick 


\begin{abstract}
The aim of this study was to elucidate the oxidation products of a candidate antimalarial drug, PL69, using a porphyrin system and to determine the accuracy of the oxidation products produced, as compared to what is expected in metabolism. PL69 is a reversed chloroquine (RCQ) that is active against chloroquine resistant malaria.

Porphyrin oxidation systems have been shown to mimic in vitro enzymatic metabolism reactions. PL69 and its known metabolite, PL16, were incubated with the porphyrin system, and then the oxidation products were collected and separated by HPLC. The oxidation products were characterized by NMR and mass spectrometry and compared to previous metabolism studies of PL69 with liver microsomes. The results of this research show that this porphyrin system is an acceptable mimic of in vitro metabolism methods for RCQs and provides a good framework for understanding the types of metabolism that will occur in vivo for RCQs.
\end{abstract}




\section{$\underline{\text { Acknowledgements }}$}

Many thanks to everyone who supported me while completing this daunting task. I could not have done it without the guidance of Dr. David Peyton and the rest of the Peyton lab group. I also want to thank my husband, Kyle Kendrick, for all of his support, guidance, and understanding while l've been completing this and trying to figure out my next steps. Thank you to my family, as well, for always being there for me and supporting my dreams. Lastly, thank you to the Portland State University chemistry department for providing me with the opportunity to engage in a research project such as the one described in this thesis. 


\section{$\underline{\text { Table of Contents }}$}

Acknowledgements. 


\section{$\underline{\text { Tables }}$}

Table 1: Comparison of key amino acids in CQ-sensitive and CQ-resistant strains of P. falciparum 5

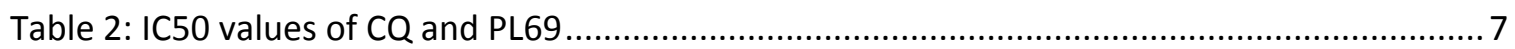

Table 3: The recommended systems for mimicking various enzymatic oxidations ..................... 14

Table 4: Percentage of metabolites found from incubation ........................................................ 16

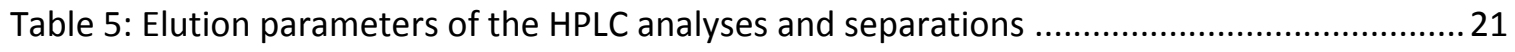

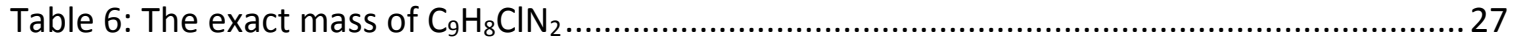

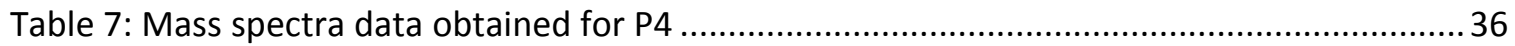




\section{Figures}

Figure 1: The life cycle of the Plasmodium parasite ................................................................. 2

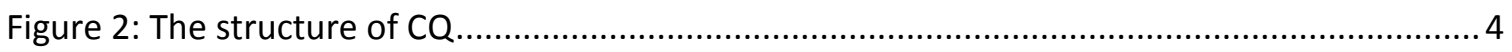

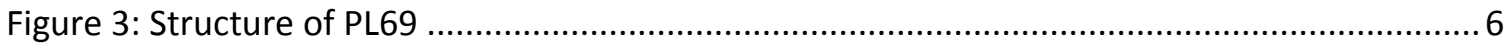

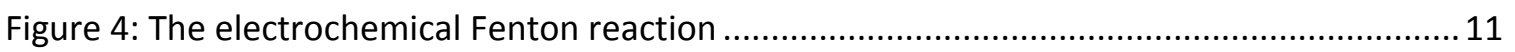

Figure 5: Structure of iron (III) tetrasodium meso-tetrakis(p-sulfonatophenyl)porphyrin ........... 13

Figure 6: Structure of PL69 - a reversed chloroquine ................................................................. 15

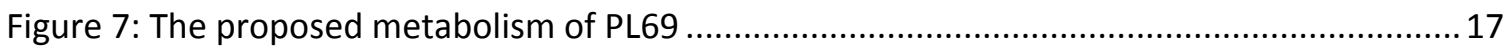

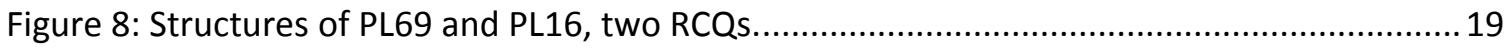

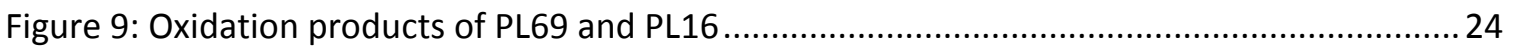

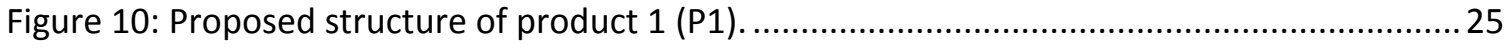

Figure 11: NMR spectra of PL69 in comparison with product 1, 4-amino-7-chloroquinoline.......26

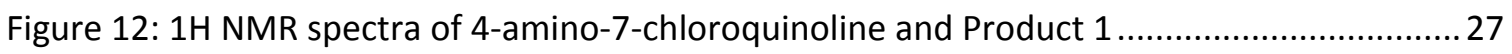

Figure 13: 1H NMR assignments of product 1 as compared to 4-amino-7-chloroquinoline..........27

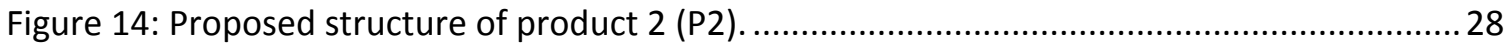

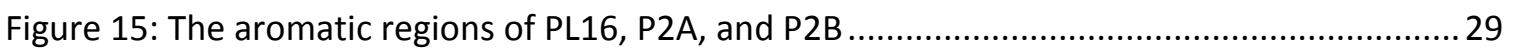

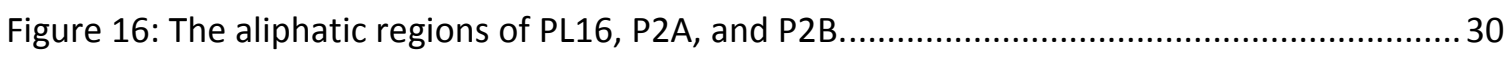

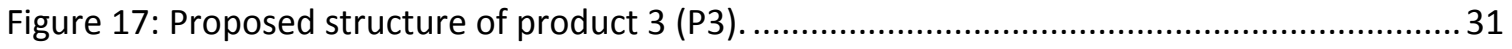

Figure 18: The structure of PL69 with the piperidine assignments ............................................. 32

Figure 19: Comparison of $1 \mathrm{H}$ NMRs of P3 with 4-hydroxypiperidine and 4-aminopiperidine ....... 33

Figure 20: Structure of P3 with letters correlating to the ${ }^{1} \mathrm{H}$ NMR shifts .................................... 33

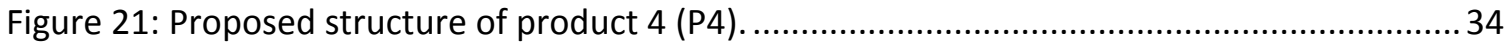

Figure 22: ${ }^{1} \mathrm{H}$ NMR of PL69 compared to P4 from three different incubations ............................. 35

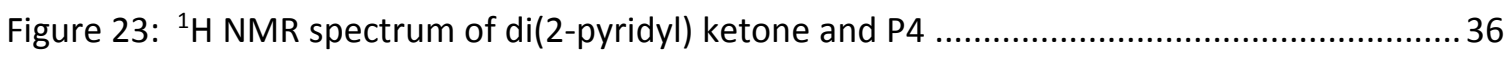

Figure 24: The chemical shifts of P4 (observed shift) as compared with di(2-pyridyl) ketone ......36

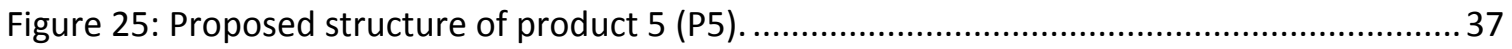

Figure 26: Comparison of ${ }^{1} \mathrm{H}$ NMR spectra of PL69 (top) and P5 (bottom).................................. 38

Figure 27: Comparison of the aliphatic region of PL69 (top) and P5 (bottom). ........................... 38

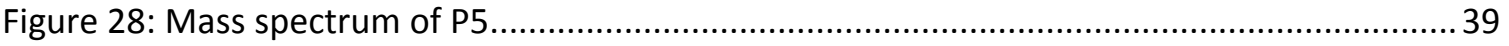

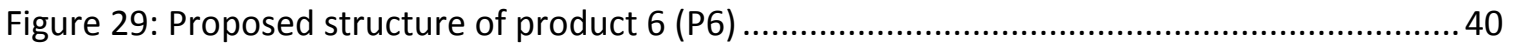


Figure 30: The top spectrum is P6 in deuterated methanol ................................................... 41

Figure 31: Possible explanation of P6 fragment measured by the MS ...................................... 42

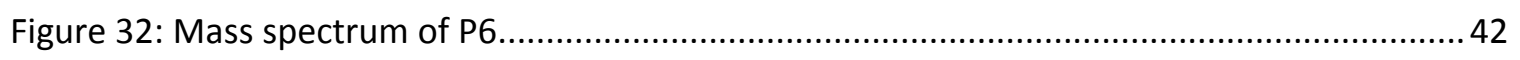

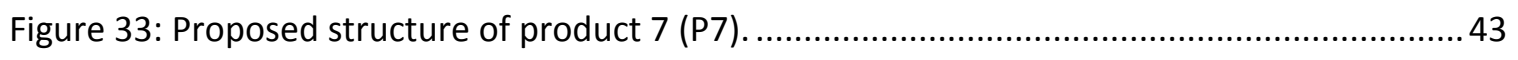

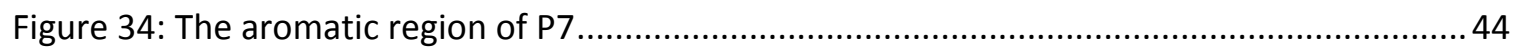

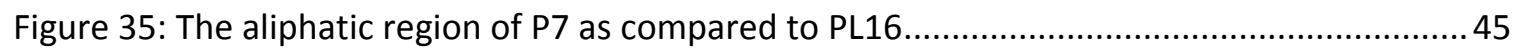

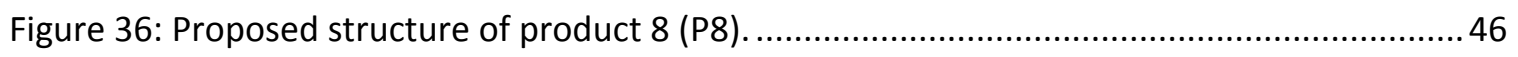

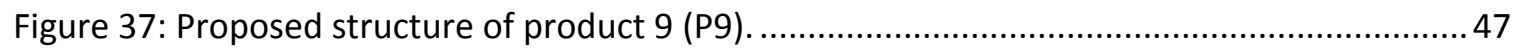

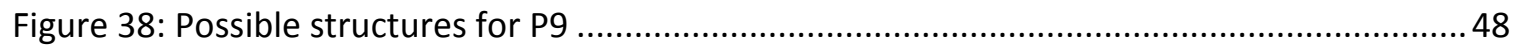

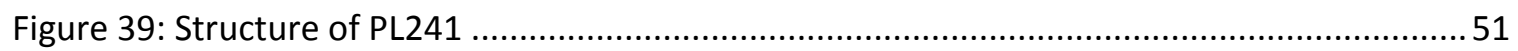

Figure 40: Comparison of the oxidation products with the metabolites determined by WuXi

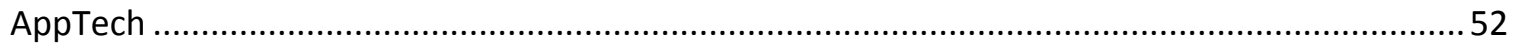




\section{$\underline{\text { Introduction }}$}

Malaria is a significant issue throughout the world, affecting the lives of many people. In 2012, 207 million people were infected with malaria, and 627,000 of those infections resulted in death. Sub-Saharan Africa is the region most affected by malaria, accounting for $90 \%$ of the deaths related to malaria ("10 Facts on Malaria", 2014). The physical effects of malaria include fevers, headaches, vomiting, disruption of blood supply, and, possibly, death (Malaria, 2014). Malaria affects a person physically, but in endemic areas, also severely affects the country's economy due to debilitating cyclic effects during infection, rendering a person unable to work consistently (Gallup, 2001). Human malaria is caused by five different species of Plasmodiae; Plasmodium ovale, malariae, knowlesi, vivax, and falciparum. P. falciparum accounts for $95 \%$ of deaths caused by malaria, and is therefore the most heavily researched (Roepe, 2009).

The malaria parasite is transmitted via the female Anopheles mosquito. When the mosquito takes a blood meal, sporozoites are injected from the mosquito into the host and go straight to the liver cells to multiply (Figure 1,\#1-2). These sporozoites then invade the host erythrocytes and go through a process of reproduction called schizogony, producing merozoites (Figure 1,\#3-4). The production of many merozoites then bursts the red blood cells allowing the merozoites to infect other erythrocytes. Merozoites that infect erythrocytes can differentiate into male and female gametocytes which can then be taken up by a mosquito (Figure 1, \#5-6). Sexual reproduction occurs 
in the mosquito forming more sporozoites to be transmitted to other hosts (Figure 1,

\#7-9) (Sherman, 2005).

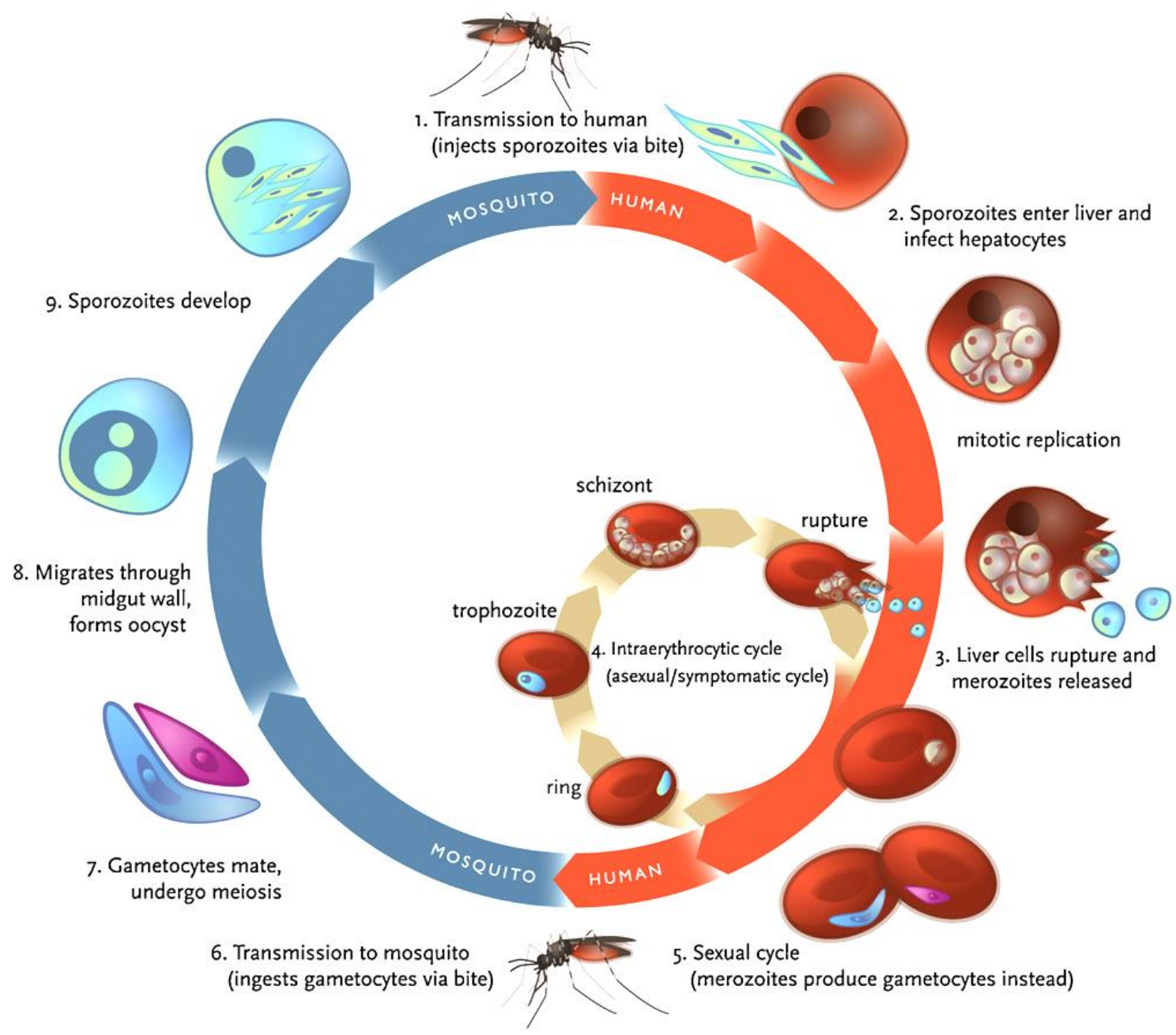

Figure 1: The life cycle of the Plasmodium parasite (Klein, 2013)

A common method of treating malaria is to inhibit Plasmodium at the merozoite stage to prevent rupturing of the erythrocytes, so the merozoites cannot further infect more erythrocytes. The parasites in the red blood cells digest hemoglobin in their digestive vacuole (DV) to obtain needed amino acids, releasing free heme 
(ferriprotoporphyrin IX) (Pishchany, 2012). Heme is toxic to the parasite because it does not have the heme oxygenase pathway, so it forms hemozoin (Roepe, 2009). Hemozoin is crystallized heme that forms in the digestive vacuole of the parasite because the $\mathrm{pH}$ of the digestive vacuole is about 5.2 and heme precipitates as hemozoin below pH 5.4 (Kuhn, 2007). As it crystallizes, hemozoin deposits in the gut of the parasite, which is the cause of the characteristic black granules in erythrocytes infected with malaria. Rupturing of the red blood cells releases the merozoites as well as hemozoin. This stage of the Plasmodium lifecycle is what causes the symptoms commonly associated with malaria.

Inhibiting formation of hemozoin has been a target for many antimalarial drug therapies, and is the method of action for one of the most widely known and used antimalarial drugs, chloroquine (CQ) (Figure 2). CQ was put in to use shortly after WWII and was very successful because it was effective, inexpensive, and safe for children and pregnant women (Wellems, 2001). CQ is active against $P$. falciparum through interactions with heme in the digestive vacuole of the parasite. There is a large accumulation of $\mathrm{CQ}$ in the digestive vacuole in part because of a $\mathrm{pH}$ gradient between the cytoplasm of the red blood cells, the cytoplasm of the parasite, and the digestive vacuole. The red blood cell and parasite cytoplasm are very similar in $\mathrm{pH}$, about 7.1. At this $\mathrm{pH}, \mathrm{CQ}$ has a +1 charge and is able to freely move through the cell membranes all the way into the digestive vacuole (Chinappi, 2010). The $\mathrm{pH}$ of the digestive vacuole is about 5, which makes CQ have a 2+ charge. Because of this large $\mathrm{pH}$ difference and 
formation of the doubly charged species, $C Q$ is able to accumulate in the DV much higher than in the cytoplasm (Kuhn, 2007). In the digestive vacuole, CQ interacts with heme, preventing the formation of hemozoin, eventually killing the parasite (Roepe, 2009). The exact method of inhibition by CQ is not known, but it is believed that CQ might affect the $\mathrm{pH}$ of the DV, therefore affecting the heme polymerization. Another idea is that CQ binds directly to heme, preventing development of a crystal (Roepe, 2009).

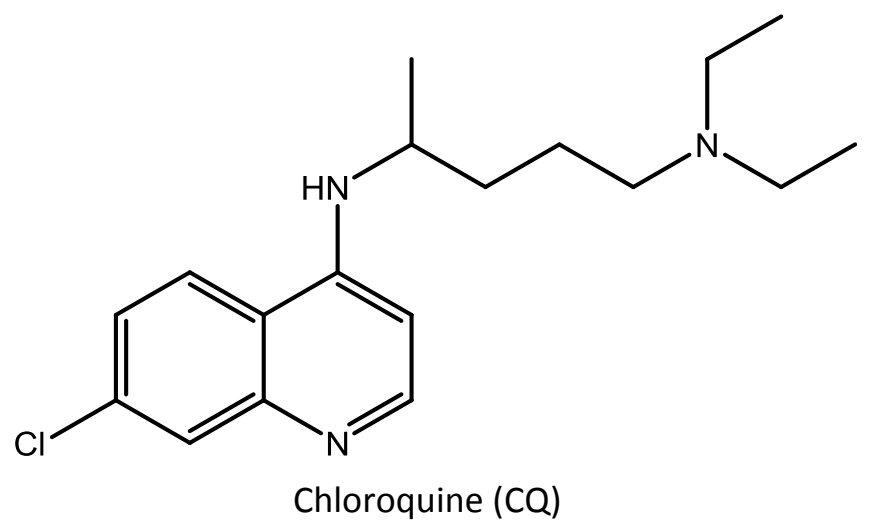

Figure 2: The structure of $\mathrm{CQ}$.

Despite the effectiveness of $C Q$, there is increasing worldwide resistance to $C Q$ as well as to many other antimalarial drugs. The digestive vacuole of the CQ-sensitive and -resistant $P$. falciparum has a membrane transporter called the $P$. falciparum chloroquine resistance transporter (PfCRT), which is partly responsible for the increasing resistance to chloroquine. Resistant parasites have been found to have a mutated version of the pfcrt gene, causing mutations in the protein sequence that allows the parasite to move CQ out of the digestive vacuole. With $C Q$ at a reduced DV 
concentration, hemozoin is once again formed allowing the parasite to survive (Chinappi, 2010).

$\mathrm{CQ}$ resistance isn't caused by just one mutation of a nucleic acid in the pfcrt gene, but by many mutations. Outlined in Table 1 are the various strains of CQ-resistant P. falciparum and the mutations of amino acids that lead to resistance. It is important to note that there isn't just one strain of CQ-resistant malaria that spread across the world, but rather resistance to CQ developed independently in different parts of the world, with different types of mutations leading to the resistance. A common mutation in all the strains listed in the table is a change from lysine at the 76 position to threonine. The CQ-sensitive strain labeled 106/1 has many of the same mutations as the resistant strands, except it doesn't have the mutation at the 76 position. This indicates that mutating the positively charged lysine to an uncharged threonine is a key mutation in resistance to CQ (Wellems, 2001).

\begin{tabular}{ll|l|llll|l|l|l|l|l} 
& \multicolumn{7}{c}{ PfCRT position and encoded amino acid } \\
\cline { 2 - 9 } \multicolumn{1}{c}{ Parasite type and origin } & 72 & 74 & 75 & 76 & 97 & 220 & 271 & 326 & 356 & 371 \\
\hline CQ-sensitive & & C & M & N & K & H & A & Q & N & I & R \\
Wild type & C & I & E & K & H & S & E & S & I & I \\
106/1 & & & & & & & & & & \\
CQ-resistant & C & I & E & T & H & S & E & S & T & I \\
SE Asia and Africa, type E1a & C & I & E & T & H & S & E & S & I & I \\
SE Asia and Africa, type E1b & S & M & N & T & H & S & Q & D & L & R \\
Papua New Guinea, type P1 & S & M & N & T & H & S & Q & D & L & R \\
South America, type W1a & C & M & N & T & H & S & Q & D & L & R \\
South America, type W1b & C & M & E & T & Q & S & Q & N & I & T \\
South America, type W2 &
\end{tabular}

Table 1: Comparison of key amino acids in CQ-sensitive and CQ-resistant strains of P. falciparum as well as where the resistant strains originated. 
The increasing resistance of $P$. falciparum to $C Q$, leads to a need for more potent, cost effective drugs that are active against this parasite. A new drug, PL69, has been synthesized that is a "reversed chloroquine" (RCQ), which combines chloroquine with a reversal agent (Figure 3). Reversal agents are molecules that reverse resistance to drugs; in this case, reversing resistance to chloroquine. Reversal agents can be taken with a drug as a cocktail or can be combined with the drug molecule to make a new drug, which is what was done in the case of PL69. The reversal agent on PL69 reduces the efflux of the chloroquine-like drug from the digestive vacuole, reversing the parasite's resistance (Burgess, 2010). The IC 50 values of CQ and PL69 for a CQ-sensitive strain and a CQ-resistant strain are listed in Table 2 . These $\mathrm{IC}_{50}$ values refer to the concentration of drug needed to inhibit growth of the Plasmodium by 50\%. PL69 has a lower $\mathrm{I} \mathrm{C}_{50}$ than $\mathrm{CQ}$ against the sensitive strain as well as has a very low $\mathrm{IC} 50$ against the resistant strain, showing that PL69 reverses CQ resistance (Andrews, 2010).

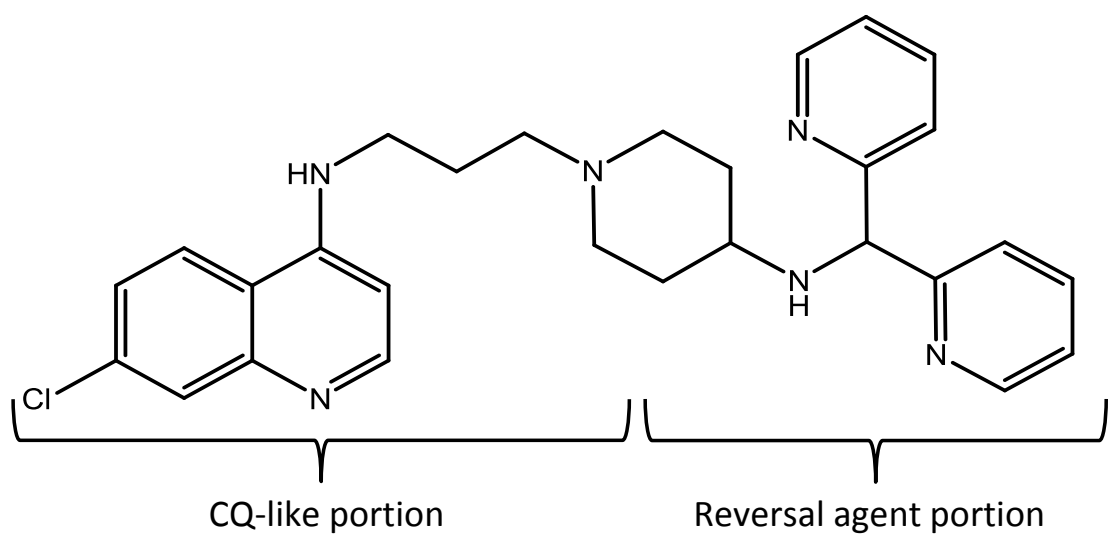

Figure 3: Structure of PL69, a reversed chloroquine, indicating the CQ-like and reversal agent portions of the molecule 


\begin{tabular}{|c|c|c|}
\cline { 2 - 3 } \multicolumn{1}{c|}{} & \multicolumn{2}{c|}{$\mathrm{IC}_{50}(\mathrm{nM})$} \\
\hline Compound & D6 (sensitive) & Dd2 (resistant) \\
\hline CQ & 7 & 102 \\
\hline PL69 & 1.6 & 1.8 \\
\hline
\end{tabular}

Table 2: IC50 values of CQ and PL69 for a CQ-sensitive and a CQ-resistant strain of P. falciparum (Andrews, 2010).

As drugs are developed, their metabolism products need to be identified quickly and early in the drug discovery process. There are multiple reasons as to why it is important to elucidate drug metabolism products, including to determine toxicity, efficacy, and pharmacokinetics of the metabolites. There are three phases in drug metabolism, commonly listed as phase I, phase II, and phase III. Phase I is mostly done by the cytochromes P450 and makes the substrate more water soluble. This usually occurs by some sort of oxidation, but can also occur through $N$-dealkylation, hydrolysis, demethylation, etc. In phase II, the oxidized substrate is conjugated to a charged species. Phase II processes include glucuronidation, sulfation, glutathione conjugation, and methylation. Phase III consists of elimination of the xenobiotic from the body (Jurva, 2003).

Human phase I drug metabolism takes place mostly in the liver, but also can occur in the digestive tract. In humans, there are 75 different isozymes of CYP450 with a large majority of these being embedded in membranes (Danielson, 2002). The liver has other enzymes involved in metabolism, but $75 \%$ of phase I drug metabolism is done 
by CYP450s. Of those CYPs, almost half of the metabolism is done by just three isozymes, CYP3A4, 5, and 7. There is a cofactor that is consistent in all CYP450s, which is iron(III)protoporphyrin IX, or heme (Woggon, 2007). This porphyrin cofactor is the reactive center of the CYP450s, producing the active species by binding to molecular oxygen. The general reaction catalyzed by CYP450 is shown in the following equation, with "SH" signifying substrate:

$$
\mathrm{NAD}(\mathrm{P}) \mathrm{H}+\mathrm{O}_{2}+\mathrm{SH}+\mathrm{H}^{+} \rightarrow \mathrm{NAD}(\mathrm{P})^{+}+\mathrm{SOH}+\mathrm{H}_{2} \mathrm{O}
$$

The role of CYP450 in drug metabolism is to activate oxygen, which can then attack the substrate, introducing more polar functionality, making it more water soluble (Danielson, 2002). The main reaction of CYP450s that is discussed in literature is hydroxylation (shown above), but there are many other reactions catalyzed by this enzyme. These other reactions include, but are not limited to, deamination, epoxidation, S-, N-, and O-dealkylations, and dehalogenations.

It is not often easy to determine the identity of metabolites, but it is important to do so. Looking at the pharmacokinetics allows the identification of what CYP is involved in metabolism to help in determining drug-drug interactions as well as knowing how fast the drug is degraded. There are multiple methods to examine metabolism, some easier and cheaper than others. Even though many of the products are the same, they are produced in various amounts and sometime there are more or less metabolites 
depending on the method. The next section will discuss the most common types of in vitro metabolism as well as chemical mimics of enzymatic oxidations. 


\section{Mimicking in vivo Metabolism}

There are many new drug candidates being synthesized every day and in order for the drug to advance in clinical trials, the metabolites need to be identified and any toxicity made to be evaluated. There are a few ways of identifying metabolites, but the current methods usually include using hepatocytes or liver microsomes. Microsomes are vesicle-like structures formed upon homogenization of the liver and differential high-speed centrifugation. During homogenization and centrifugation, the CYP450 enzymes become very concentrated in these vesicles, making them desirable for analysis of metabolites (Baranczewski, 2006). Hepatocytes and microsomes give fairly accurate in vitro depictions of the type of transformations the drug will undergo. However, there are some downsides to using these methods. Both hepatocytes and microsomes must be extracted from an organism and kept frozen, leading to a high cost for every drug incubation. Another downside of using these in vitro methods is that the enzymes don't remain active for very long and it is often difficult to obtain enough product for complete analysis of the metabolites.

In order to minimize costs of examining the metabolites formed, there has been an increasing amount of research on chemical mimics of CYP450 systems. These mimics include an electrochemical system using the Fenton reaction, and using metalloporphyrins to mimic the heme cofactor present in CYP450s. The Fenton reaction involves using an electrochemical cell to reduce $\mathrm{Fe}^{3+}$ to $\mathrm{Fe}^{2+}$, which then reacts with 
hydrogen peroxide, generating hydroxyl radicals and $\mathrm{Fe}^{3+}$ (Figure 4). The electrochemical cell then reduces the iron, regenerating $\mathrm{Fe}^{2+}$ and starting the oxidation process over. The hydroxyl radicals can add to aromatic rings and double bonds causing oxidation such as what would be seen with CYP450 (Johansson, 2007).

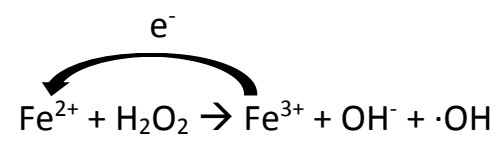

Figure 4: The electrochemical Fenton reaction

A study to compare electrochemical oxidation products with those formed in CYP450 reactions was done by Ulrik Jurva (Jurva, 2003). The results of that study showed that the products of the electrochemical oxidation were often the same as those seen in the enzyme catalyzed reactions. The reactions that were found to work by this method were one-electron oxidations such as $\mathrm{N}$-dealkylation, alcohol oxidation, and dehydrogenation. Hydrogen atom abstraction reactions had too high of oxidation potentials, and include reactions such as O-dealkylation and hydroxylation of unsubstituted aromatic rings. Even though the method using electrochemical oxidation does not fully mimic the type of oxidations of the CYP450 enzymes, it is a good model to examine oxidation products to propose the type of products that may be formed by microsomes or hepatocytes (Jurva, 2003).

Another model system to study drug oxidation involves mimicking part of the active site of CYP450s, rather than just mimicking their oxidation reactions. The active site of CYP450 has an iron protoporphyrin IX, which creates active species of oxygen that cause the oxidation processes. Research is ongoing using metalloporphyrins as 
mimics of the CYP450 active site, heme. The most useful CYP-mimicking porphyrins are water soluble metalloporphyrins, which can more closely mimic the environment in which metabolism occurs, as compared to metalloporphyrins that are only soluble in organic solvents (Bernadou, 1990). Metalloporphyrins have the same benefits as the electrochemical systems, as compared to microsomes and hepatocytes, except the system does not regenerate itself. Research has been done on various types of metalloporphyrins, including changing the metal ions and attaching different groups to the porphyrins (Johansson, 2007).

There are quite a few instances showing that metalloporphyrin oxidations can mimic CYP reactions when added in conjunction with an oxygen donating compound, such as hydrogen peroxide. A study done by J. Bernadou compared the activity of various metalloporphyrins to horse radish peroxidase (HRP), which has a heme group in the active site (Bernadou, 1990). Four metalloporphyrins were used: iron(III) and manganese (III) tetrasodium meso-tetrakis(p-sulfonatophenyl)porphyrin [Fe(III)TPPS (Figure 5) and Mn(III)TPPS, respectively], and iron(III) and manganese(III) tetraacetate meso-tetrakis(4- $\mathrm{N}$-methylpyridiniumyl)porphyrin [Fe(III)TMPyP and Mn(III)TMPyp, respectively]. Oxidation of acetaminophen by these four metalloporphyrins and HRP were examined, because the oxidation product of acetaminophen is known. The metalloporphyrins and HRP all produced the desired metabolite, $N$-acetyl- $p$ benzoquinone-imine (NAPQI) with varying rates and yields. Both Fe(III)TPPS and HRP had initial turnover rates that were too fast to be measured. The amount of NAPQI 
produced by Fe(III)TPPS ( $88 \%$ yield) was higher than the other metalloporphyrins, as well as higher than HRP (40-50\% yield). The other metalloporphyrins transformed between 20 and $50 \%$ of acetaminophen to NAPQI, which at the high range is comparable to the amount of product produced by HRP (Bernadou, 1990). This study is a good indicator of how metalloporphyrins can be mimics of an oxidizing enzyme.

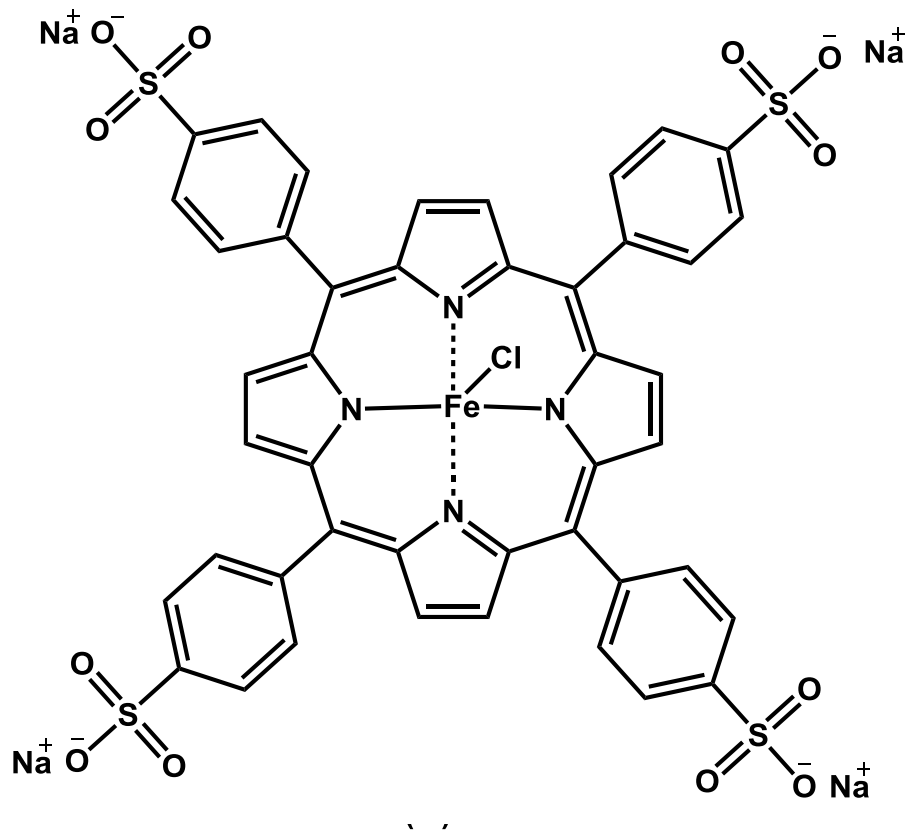

Figure 5: Structure of iron (III) tetrasodium meso-tetrakis(p-sulfonatophenyl)porphyrin.

Another study compared the products produced by oxidizing metoprolol with human and rat liver microsomes, the electrochemical Fenton reaction, and a metalloporphyrin system using Fe(III)TPPS (Figure 5) (Johansson, 2007). The products were examined using liquid chromatography coupled with a mass spectrometer (LCMS). Looking at the LC chromatograms, there were seven products produced by rat liver microsomes, and four produced by human liver microsomes. The electrochemical 
Fenton reaction produced five of the same products as the rat microsomes and the porphine system produced six of the seven products. The products were also analyzed by mass spectrometry in order to be sure the peaks seen in the chromatograms were the same. Between each method, there was variation in the amounts of products produced, but both methods formed the major metabolites seen in the microsome systems. This research group analyzed the oxidation products of other drugs to compare the products of each system, as well as to determine the mimic system that best executed each type of reaction, the results of which are shown in Table 3. The porphine system was recommended as a mimic for many of the enzymatic oxidations, including N-dealkylation, alcohol oxidation, and aliphatic hydroxylation (Johansson, 2007).

\begin{tabular}{|c|c|}
\hline Enzymatic Oxidation & Recommended System \\
\hline Aliphatic hydroxylation & EC-Fenton or porphine \\
\hline Benzylic hydroxylation & EC-Fenton or porphine \\
\hline Aromatic hydroxylation & EC-Fenton or porphine \\
\hline N-dealkylation & EC-Fenton or porphine \\
\hline N-oxidation & Porphine \\
\hline O-dealkylation & Porphine \\
\hline Alcohol oxidation & Porphine \\
\hline Aldehyde oxidation & Porphine \\
\hline Deydrogenation & EC-Fenton or porphine \\
\hline
\end{tabular}

Table 3: The recommended systems for mimicking various enzymatic oxidations (Johansson, 2007). 
While the electrochemical system seems effectively to mimic enzymatic oxidation, there is more equipment needed to use it. The easier use of metalloporphyrins and more readily available equipment led to the use of Fe(III)TPPS for the research done in this thesis. Rat liver microsomes were also used to compare the products of metabolism to the oxidative products from the porphine system.

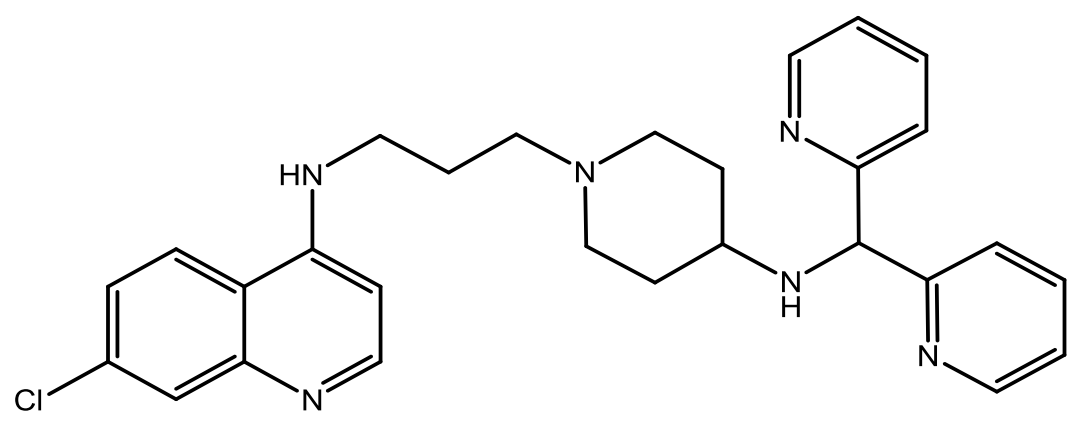

Figure 6: structure of PL69 - a reversed chloroquine

Based on the literature of the metalloporphyrins oxidations, it was hypothesized that there could be N-dealkylation at the amine linkages in PL69 (Figure 6), aromatic hydroxylation, or hydroxylation on the aliphatic carbon chain or piperidine. A metabolic study was done on PL69 by WuXi AppTec, but the only type of analysis done on the products was LC-MS. In order to fully understand the products formed, NMR analysis needed to be done, which was the main method of elucidation used in this thesis. The incubations done with both methods had to be done in sufficiently large quantities in order to obtain enough product formation to be analyzed by NMR.

The metabolism of PL69 in human, rat, and liver microsomes was analyzed through mass spectrometry by WuXi AppTec, a bio analytical company based in China. Figure 7 shows the proposed structures for the metabolites produced in the highest 
amounts. The percent formation in human liver microsomes is listed after the molecular weights. Table 4 lists the percent formation of each metabolite in rat, monkey, and human liver microsomes. Many of the proposed metabolism products are results of $\mathrm{N}$-dealkylation and/or oxygenation. $\mathrm{M} 1$ and $\mathrm{M} 3$ were produced in the highest amounts in the human microsomes, $14.26 \%$ and $21.30 \%$, respectively, as measured by LCMS. M1 and M3 were also produced in high amounts in the rat and monkey microsomes (Table 4). The relative abundance was calculated by the areas of the parent and metabolite peaks on the mass spectrum. There were some metabolites not produced in the human microsomes that were produced in the either the monkey or rat microsomes, but many of the high yield metabolites were produced with the microsomes of all three species. Mass spectrometry provides an accurate mass and general picture of what the molecule might look like based on the mass, but without other structural analysis, mostly by NMR, the complete structures will not be known.

\begin{tabular}{|c|c|c|c|}
\hline \multirow{2}{*}{ Metabolite } & \multicolumn{3}{|c|}{ Relative Abundance (\%) } \\
\cline { 2 - 4 } & human liver microsomes & $\begin{array}{c}\text { monkey liver } \\
\text { microsomes }\end{array}$ & rat liver microsomes \\
\hline M1 & 14.26 & 7.18 & 15.97 \\
\hline M2 & 8.20 & 3.26 & 18.95 \\
\hline M3 & 21.30 & 36.20 & n/a \\
\hline M4 & n/a & 1.22 & 0.83 \\
\hline M5 & 8.40 & 5.05 & $n / a$ \\
\hline M6 & 5.80 & 3.68 & 4.25 \\
\hline M7 & n/a & 1.91 & 4.58 \\
\hline M8 & 6.48 & 10.48 & $\mathrm{n} / \mathrm{a}$ \\
\hline M9 & 0.85 & n/a \\
\hline
\end{tabular}

Table 4: Percentage of metabolites found from incubation with rat, monkey, and human liver microsomes. This data was determined by WuXi AppTec. Structures of these metabolites are in Figure 7. 


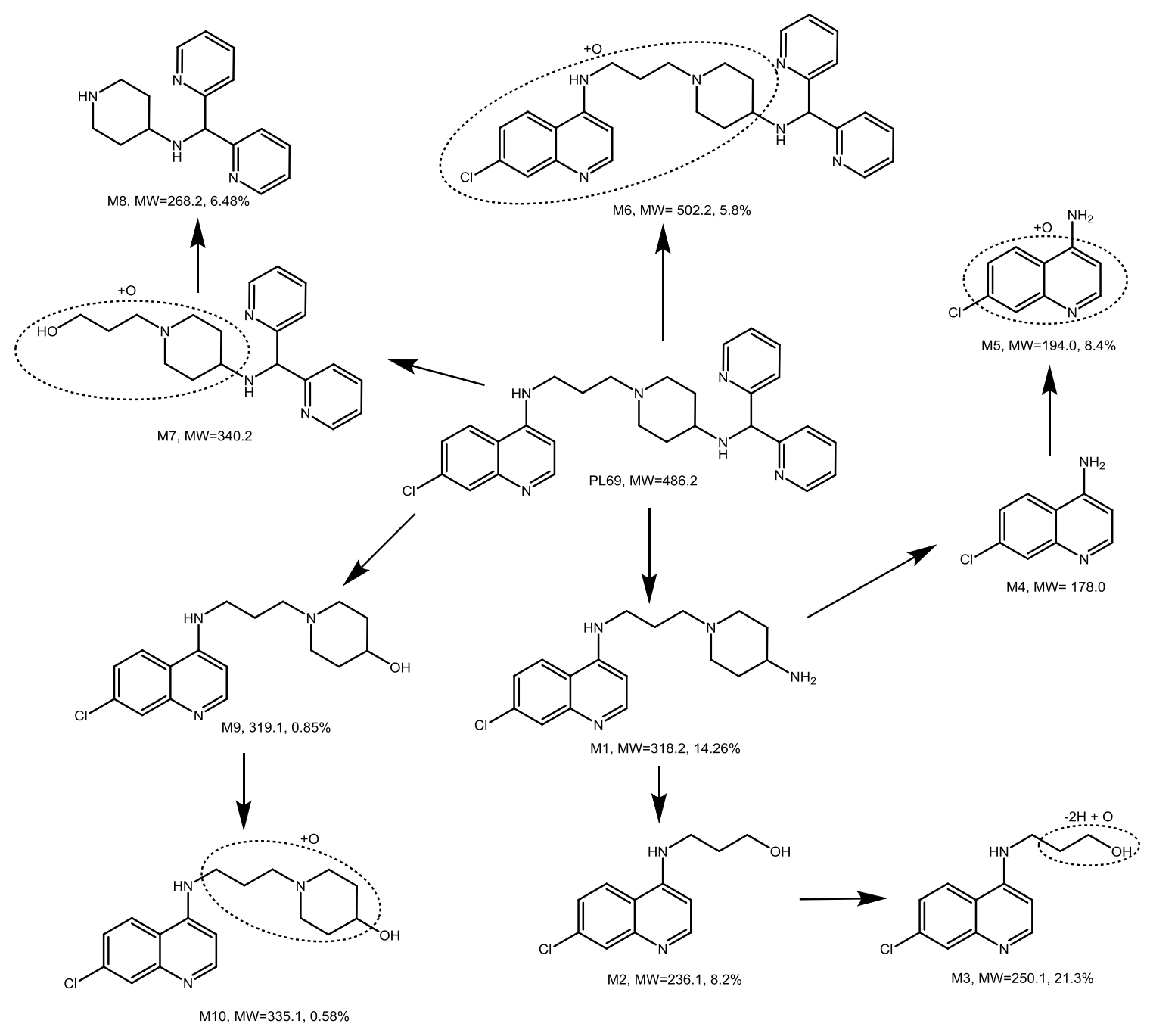

Figure 7: The proposed metabolism of PL69 using human liver microsomes. The metabolite molecular weight and the percentage formed are listed under the structures. 


\section{Methods}

The antimalarial compound, PL69 (Figure 8), was analyzed using microsomes and iron(III)meso-tetrakis(p-sulfonatophenyl)porphyrin (Fe(III)TPPS) to examine the oxidations that mimic in vivo metabolic reactions. The resulting products were separated and analyzed using HPLC, NMR, and mass spectrometry.

Microsomal Incubations -Rat liver microsomes and an NADPH regenerating system were purchased from BD Biosciences. The rat liver microsomes were harvested from male Sprague-Dawley rats and were in a concentration of $20 \mathrm{mg} / \mathrm{mL}$ in a $250 \mathrm{mM}$ sucrose solution. The NADPH regenerating system consisted of two solutions, Solution A (26 mM NADP +66 mM glucose-6-phosphate, and $66 \mathrm{mM} \mathrm{MgCl}_{2}$ in $\mathrm{H}_{2} \mathrm{O}$ ) and Solution $\mathrm{B}$ (40 $\mathrm{U} / \mathrm{mL}$ glucose-6-phosphate dehydrogenase in $5 \mathrm{mM}$ sodium citrate). The method for doing the microsomal incubations was adapted from the procedure provided by BD Biosciences upon purchase of the microsomes.

There was a small scale incubation $(0.5 \mathrm{~mL}$ total) of PL69 with the microsomes and then the method was scaled up. The large scale method consisted of the following final concentrations (final volume of $5 \mathrm{~mL}$ ): 50 uM PL69, $100 \mathrm{mM}$ phosphate buffer (pH 7.4), $0.5 \mathrm{mg} / \mathrm{mL}$ rat liver microsomes, $20 \mathrm{x}$ dilution of $\mathrm{NADPH}$ solution $\mathrm{A}$, and $100 \mathrm{x}$ dilution of NADPH solution B. Everything but the microsomes were added, which were added after the solution was in a $37^{\circ} \mathrm{C}$ water bath for 5 minutes. The solution was then incubated for 60 minutes in the $37^{\circ} \mathrm{C}$ water bath. After 60 minutes, the solution was then put on 
ice, and ice cold acetonitrile was added to halt the enzyme activity and precipitate the proteins. The solution was then centrifuged and the supernatant collected. The products were extracted by raising the $\mathrm{pH}$ to above 10 and extracting with dichloromethane (DCM).

Porphyrin incubations - Porphyrin incubations were done on both PL69 and PL16 (Figure 8), because PL16 is known from the microsomal incubations as well as other studies to be a metabolite of PL69. Thus, incubating PL16 would show whether further metabolism would occur.
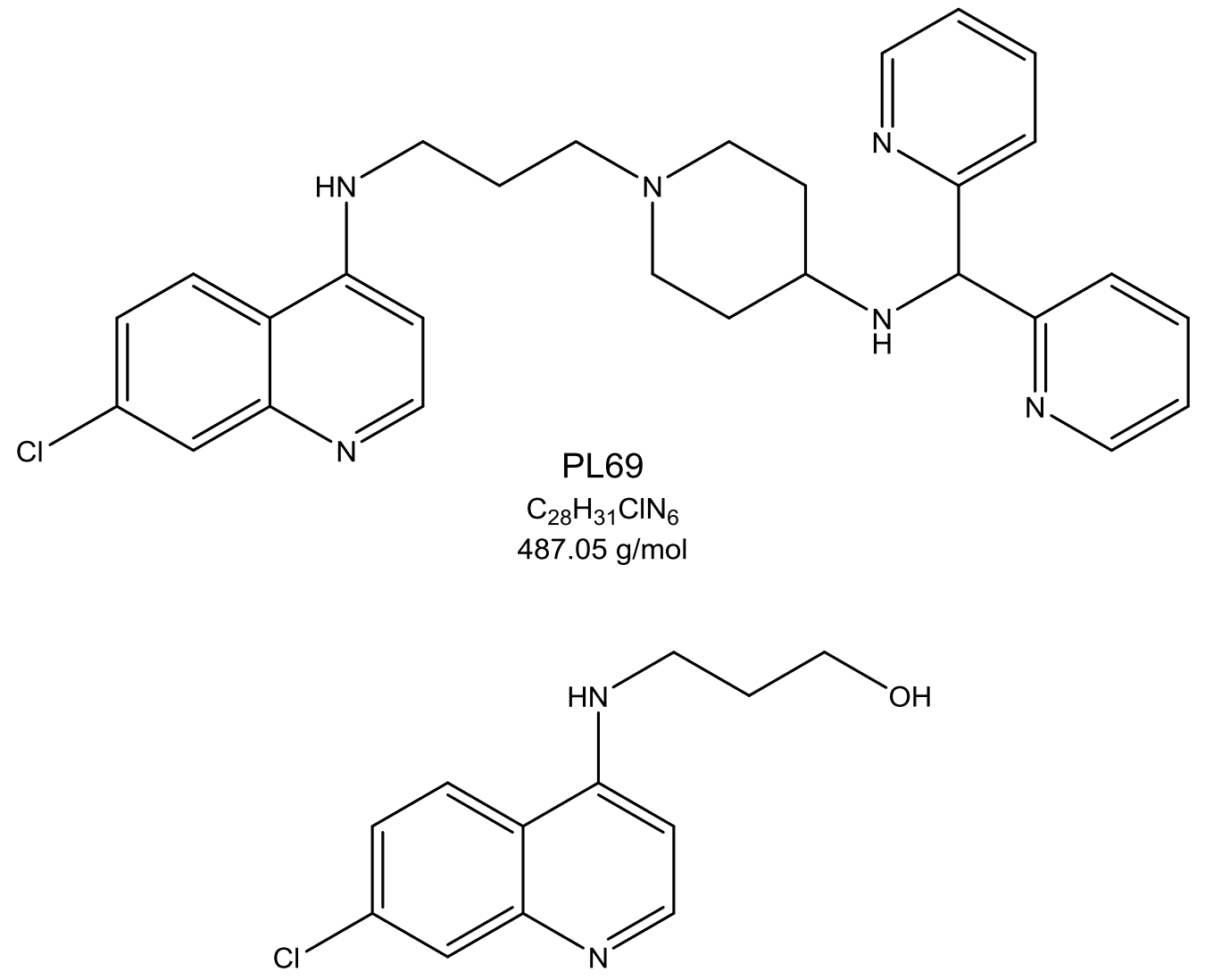

PL16

$\mathrm{C}_{12} \mathrm{H}_{13} \mathrm{CIN}_{2} \mathrm{O}$

$236.70 \mathrm{~g} / \mathrm{mol}$

Figure 8: Structures of PL69 and PL16, two RCQs. 
PL69 porphyrin incubations - Two PL69 incubations were conducted at varying incubation lengths, 30 or 60 minutes. The incubation solution before adding the hydrogen peroxide consisted of $70 \mathrm{mM}$ formic acid, $7.8 \%$ acetonitrile, $4.5 \mathrm{mg} / \mathrm{mL}$ PL69, and $1.5 \mathrm{mg} / \mathrm{mL}$ Fe(III)TPPS for the 60 minute incubation, and $6.6 \mathrm{mg} / \mathrm{mL}$ PL69 and 1.2 $\mathrm{mg} / \mathrm{mL}$ Fe(III)TPPS for the 30 minute incubation, all in a total volume of $5 \mathrm{~mL}$. This solution was placed in a foil wrapped Erlenmeyer flask, put in a $50^{\circ} \mathrm{C}$ water bath, and allowed to equilibrate for a few minutes. Hydrogen peroxide (30\%) was added to initiate the reaction making the solution, initially, $3 \%$ hydrogen peroxide. The solution was incubated for 30 or 60 minutes with intermittent stirring. Sodium hydroxide (100 $\mathrm{mM}$ ) was added to bring the solution to above $\mathrm{pH}$ 10. Dichloromethane (DCM) was used to extract the products from the solution. The DCM was removed using a rotary evaporator. A drying agent as not used with the DCM because the products were run through the HPLC for purification and didn't need to be completely pure at this point. PL16 porphyrin incubation - The incubation solution before adding the hydrogen peroxide consisted of $70 \mathrm{mM}$ formic acid, $7.8 \%$ acetonitrile, $5.6 \mathrm{mg} / \mathrm{mL}$ PL16, and 1.8 $\mathrm{mg} / \mathrm{mL}$ Fe(III)TPPS in distilled water. This solution was placed in a foil wrapped Erlenmeyer flask, put in a $50^{\circ} \mathrm{C}$ water bath, and allowed to equilibrate for a few minutes. Hydrogen peroxide (30\%) was added and the solution was incubated for 45 minutes with intermittent stirring. The same method of extraction as used for the PL69 porphyrin incubations was used for the PL16 incubations. 
High performance liquid chromatography - HPLC was used to do initial analysis of the incubation solutions as well as to separate the products. The HPLC used was a dual pump Varian Prostar 210 solvent delivery module model, and the processing software was Galaxie Chromatography. Attached was a Varian Prostar 325 dual wavelength UV/VIS detector, set at 254 and $280 \mathrm{~nm}$. A Varian Prostar 701 fraction collector was attached to the HPLC system in order to collect the fractions of interest. The solvents used were HPLC grade water and acetonitrile, both with $0.1 \%$ formic acid. The column used was a reversed phase Ascentis $C 18,150 \times 4.6 \mathrm{~mm}, 5 \mathrm{um}$. The gradient used for analysis is outlined in Table 5.

\begin{tabular}{|c|c|c|c|}
\hline Time $(\min )$ & \% A & \% B & Flow rate $(\mathrm{mL} / \mathrm{min})$ \\
\hline 0 & 95 & 5 & 1.25 \\
\hline 2 & 95 & 5 & 1.25 \\
\hline 10 & 35 & 65 & 1.25 \\
\hline
\end{tabular}

Table 5: Elution parameters of the HPLC analyses and separations. A= water with $0.1 \%$ formic acid. $B=$ acetonitrile with $0.1 \%$ formic acid. For separating the products, the gradient was modified depending on how close the peaks were together and where they eluted, but the column and solvents remained the same. The fraction collector was set to collect each fraction for 30 seconds.

\section{Analysis}

HPLC- Purity of the fractions obtained from the HPLC were analyzed using the HPLC.

The retention times were also examined to determine the peaks that were the same products.

NMR Spectroscopy- Structural analysis was done using a Bruker $400 \mathrm{MHz}$ nuclear magnetic resonance (NMR) spectrometer, a Bruker $600 \mathrm{MHz}$ NMR was also used in some experiments. 
Mass Spectrometry- Mass analysis was done using the ThermoElectron LTQ-Orbitrap Discovery high resolution mass spectrometer at the Portland State University BioAnalytical Mass Spectrometry Facility. 


\section{$\underline{\text { Results }}$}

There were many products formed in both the microsome and porphyrin methods used. This was evidenced by the many peaks present when the sample was run on the HPLC as well as multiple species in NMR spectra and mass spectra. There weren't high enough quantities of all of the products to collect and obtain good spectra on the NMR, but the 9 products produced with the highest yields were analyzed by NMR spectroscopy and mass spectrometry. These nine proposed oxidation products are in

Figure 9 on the next page. The elucidation of the each individual product is explained on the following pages. Some of the products were not fully elucidated due to low yields or loss during purification, but the base structure for each product was determined. 
<smiles>CCCCCCNc1ccnc2cc(Cl)ccc12</smiles>

P669
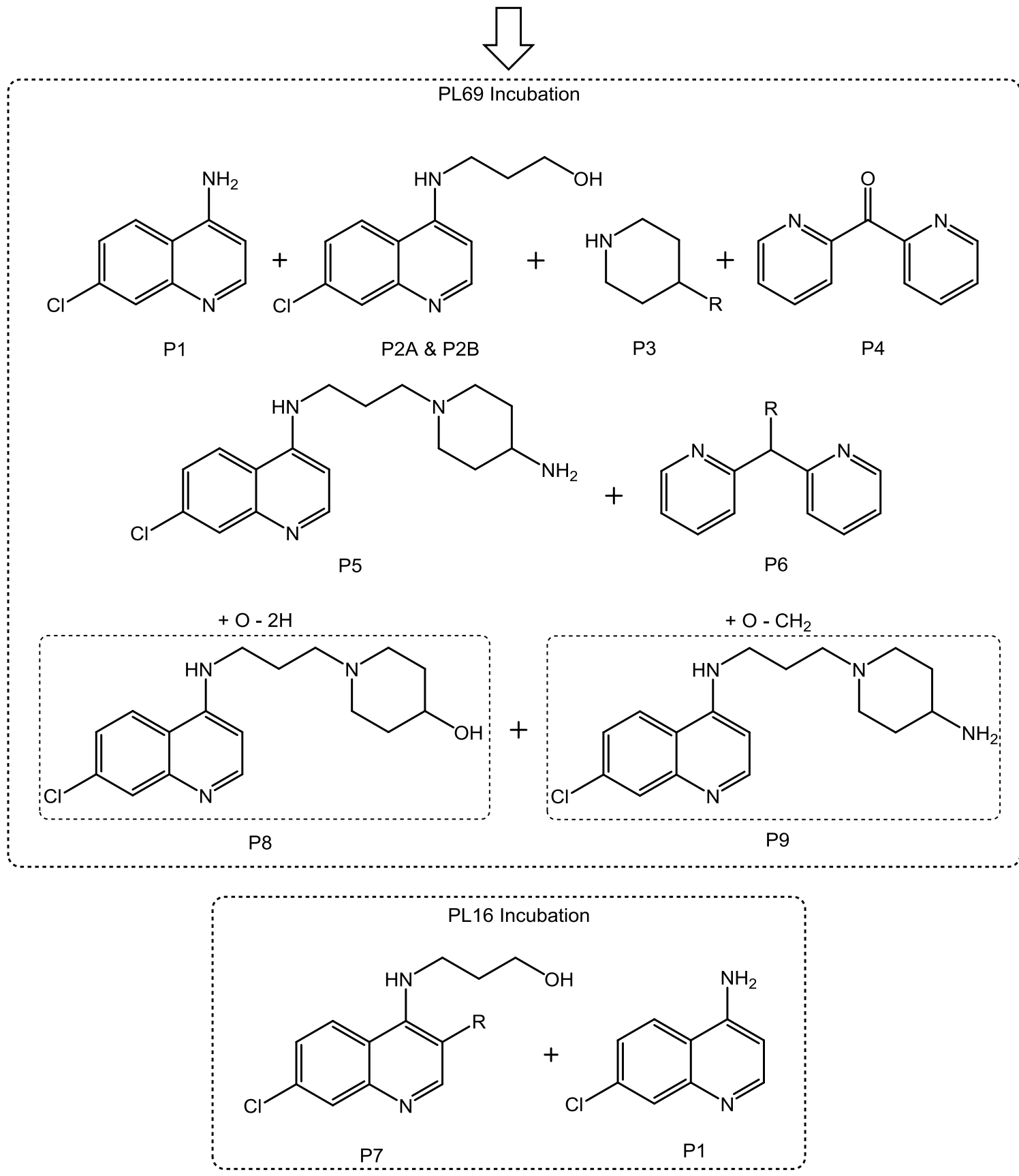

Figure 9: Oxidation products of PL69 and PL16 obtained by incubation with rat liver microsomes or a porphyrin oxidation system. Products were elucidated by NMR and mass spectrometry. 


\section{Product 1}

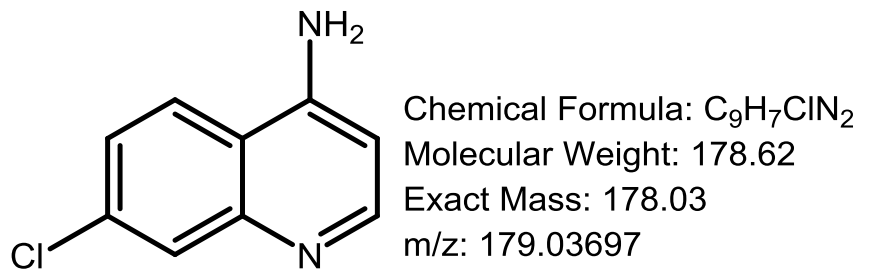

Figure 10: Proposed structure of product 1 (P1).

Product 1 (P1) was present in all of the porphyrin incubations using both PL69 and PL16. P1 was produced in three incubations, and the ${ }^{1} \mathrm{H}$ NMR spectrum as compared to PL69 is shown in Figure 11. The top spectrum is PL69 and the arrows point to the corresponding quinoline peaks in the bottom spectra. As seen from these ${ }^{1} \mathrm{H}$ NMR spectra, all five of the quinoline peaks are present in $\mathrm{P} 1$ with fairly similar chemical shifts to each other as well as to the PL69 quinoline peaks. There were no peaks in the aliphatic region of this product, indicating that the product was composed of hydrogens solely from the quinoline ring. From the NMR data, it was hypothesized that P1 was 4amino-7-chloroquinoline. Figure 13 lists the average chemical shifts of the P1 obtained from the three incubations as compared to 4-amino-7-chloroquinoline. The average was taken because this product was obtained a few times and in each NMR spectrum had a slightly different shift. The chemical shifts and the splitting of the peaks are very similar to actual 4-amino-7-chloroquinoline (Figure 12 and 13).

Mass spectrometry data was collected for product 1 in each of the three incubations to confirm the identity, which is listed in table 6 . The theoretical exact mass for the chemical formula with one hydrogen added from using positive $\mathrm{ESI}\left(\mathrm{C}_{9} \mathrm{H}_{8} \mathrm{CIN}_{2}\right)$ 
was 179.03760. The product 1 from the three incubations had $\mathrm{m} / \mathrm{z}$ values very close to the theoretical exact mass, with acceptable ppm differences. Therefore, it was confirmed that product 1 is 4-amino-7-chloroquinoline.

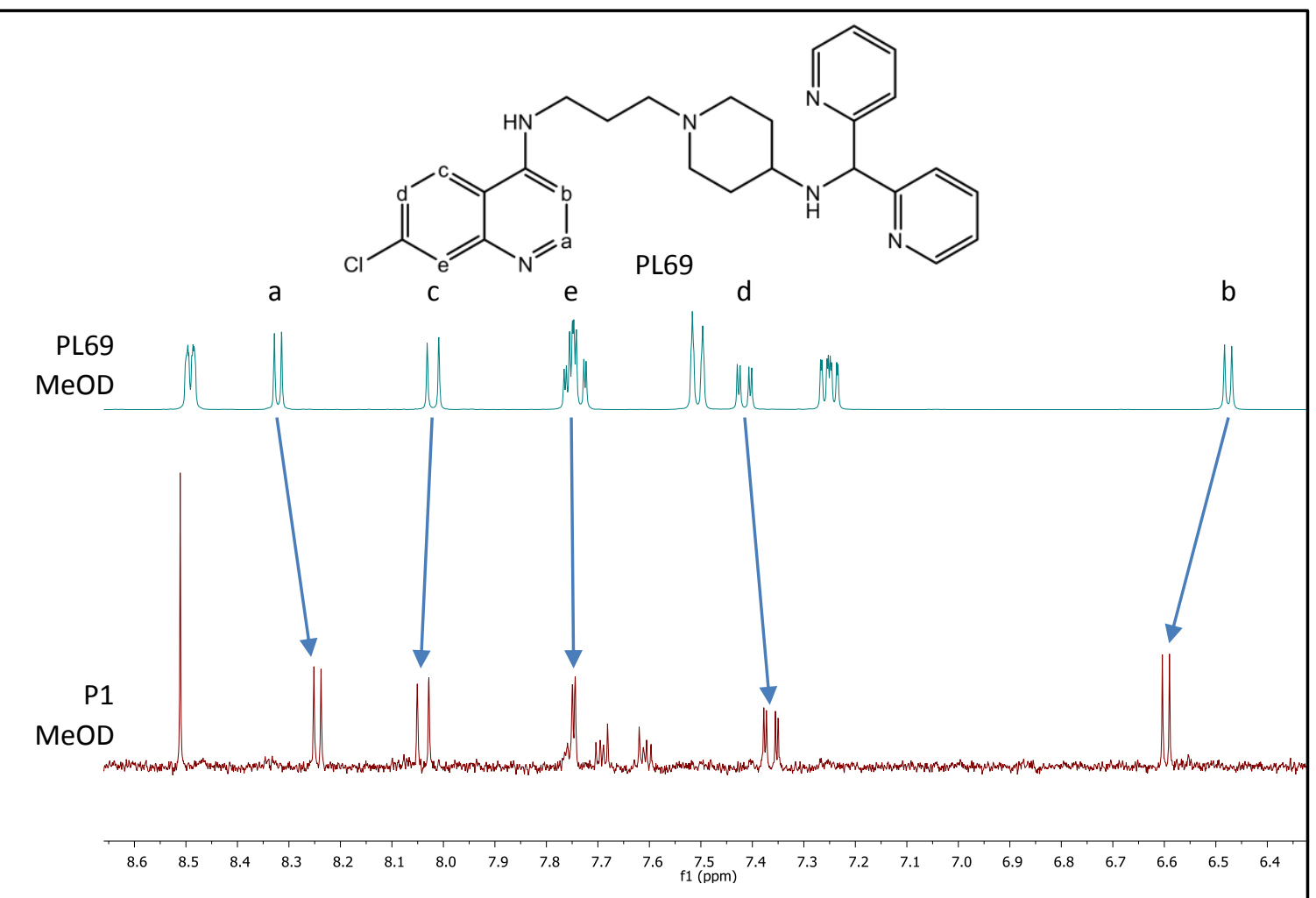

Figure 11: NMR spectra of PL69 in comparison with product 1, 4-amino-7-chloroquinoline. The quinoline hydrogen peaks of PL69 are indicated with letters corresponding to the structure of PL69. The arrows show the corresponding hydrogens in the quinoline ring. 
4-amino-7-chloroquinoline MeOD

P1

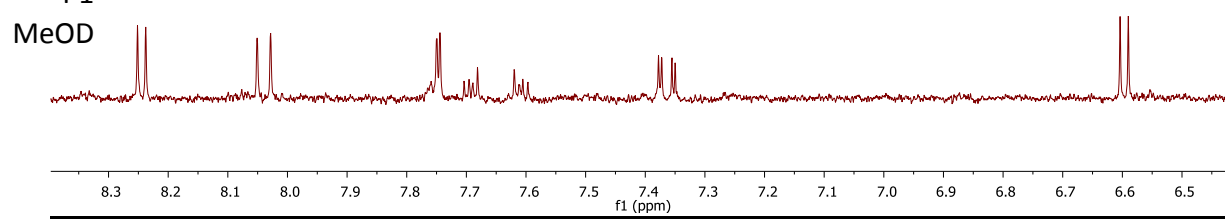

Figure 12: $1 \mathrm{H}$ NMR spectra of 4-amino-7-chloroquinoline and Product 1. The peaks of P1 are shifted fairly similar to 4-amino-7-chloroquinoline. There are two peaks at 7.61 and 7.68 ppm that are impurities that are also present in other samples.

\begin{tabular}{|c|c|c|c|c|c|}
\hline Position & $\begin{array}{c}\text { Average } \\
\text { shift (ppm) }\end{array}$ & $\begin{array}{c}\text { Theoretical } \\
\text { shift (ppm) }\end{array}$ & Integration & Multiplicity \\
\hline $\mathrm{a}$ & 8.25 & 8.27 & 1 & $\mathrm{~d}$ \\
\hline $\mathrm{b}$ & 6.72 & 6.61 & 1 & $\mathrm{~d}$ \\
\hline $\mathrm{c}$ & 8.15 & 8.07 & 1 & $\mathrm{~d}$ \\
\hline $\mathrm{d}$ & 7.54 & 7.39 & 1 & $\mathrm{dd}$ \\
\hline $\mathrm{e}$ & 7.82 & 7.77 & 1 & $\mathrm{~d}$ \\
\hline
\end{tabular}

Figure 13: $1 \mathrm{H}$ NMR assignments of product 1 as compared to 4-amino-7-chloroquinoline. The structure on the left shows the assignment of the hydrogens.

\begin{tabular}{|c|c|c|}
\hline Source of Product & Obtained $\mathrm{m} / \mathrm{z}$ & $\Delta \mathrm{ppm}$ \\
\hline theoretical $\mathrm{C}_{9} \mathrm{H}_{8} \mathrm{CIN}_{2}$ & 179.03760 & $\mathrm{n} / \mathrm{a}$ \\
\hline PL69 with Fe(III)TPPS (1) & 179.03702 & 3.2451 \\
\hline PL69 with Fe(III)TPPS (2) & 179.03619 & 7.8810 \\
\hline PL16 with Fe(III)TPPS & 179.03687 & 4.0829 \\
\hline
\end{tabular}

Table 6: The exact mass of $\mathrm{C}_{9} \mathrm{H}_{8} \mathrm{CIN}_{2}$ is listed as well as the $\mathrm{m} / \mathrm{z}$ values obtained for product 1 that was collected from the three different incubations. The $\Delta \mathrm{ppm}$ represents the difference between the obtained product $\mathrm{m} / \mathrm{z}$ and the theoretical exact mass, divided by the theoretical mass. 


\section{Product 2}

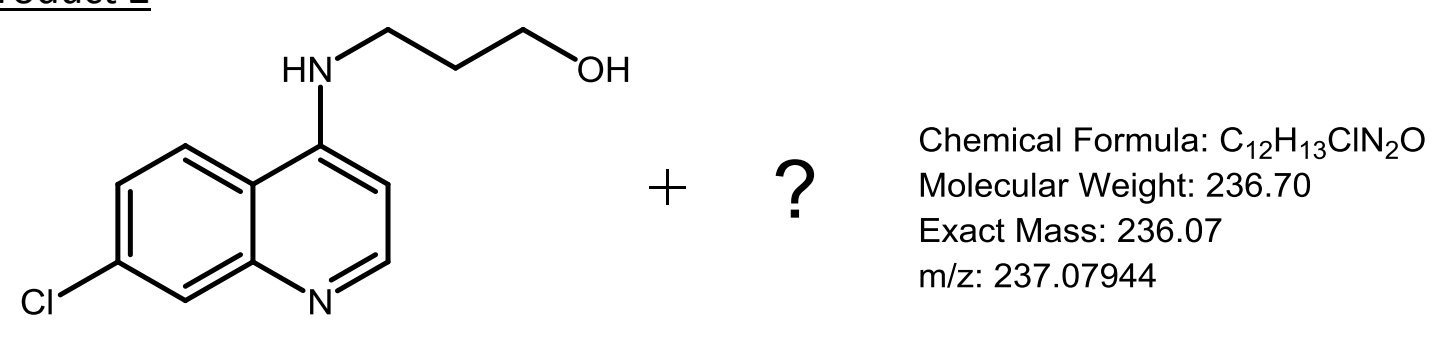

Figure 14: Proposed structure of product 2 (P2).

Product 2 (P2) is actually two products that were determined from the microsomal incubations. Both have ${ }^{1} \mathrm{H}$ NMR spectra similar to an already known compound, PL16. The two products will be referred to as P2A and P2B. These products were the few isolated compounds from the microsomal incubations. Shown in Figure 15 and 16 are the spectra for PL16 (top), P2A (middle), and P2B (bottom). In the aromatic region, P2A and P2B have slightly different shifts from PL16, but very similar shifts to each other and the same type of splitting in present. In the aliphatic region (Figure 16), the same shaped peaks are present, but are at different chemical shifts. The quartet seen at $4.11 \mathrm{ppm}$ is the methylene peak of ethyl acetate. PL16 and P2A have very similar shifts for the two triplets and the pentet. P2B, however, has one triplet shifted from $3.74 \mathrm{ppm}$ to $4.24 \mathrm{ppm}$ and the pentet is shifted from $1.98 \mathrm{ppm}$ to $2.11 \mathrm{ppm}$. It is hypothesized that P2A is PL16 and P2B is a molecule similar to PL16, but possibly with a different electron withdrawing group that causes hydrogen " $\mathrm{h}$ " to shift farther downfield. The shift changes in the aromatic region could possibly be accounted for by $\mathrm{pH}$ differences between the samples. The aromatic protons seem to be highly influenced by slight pH changes. 

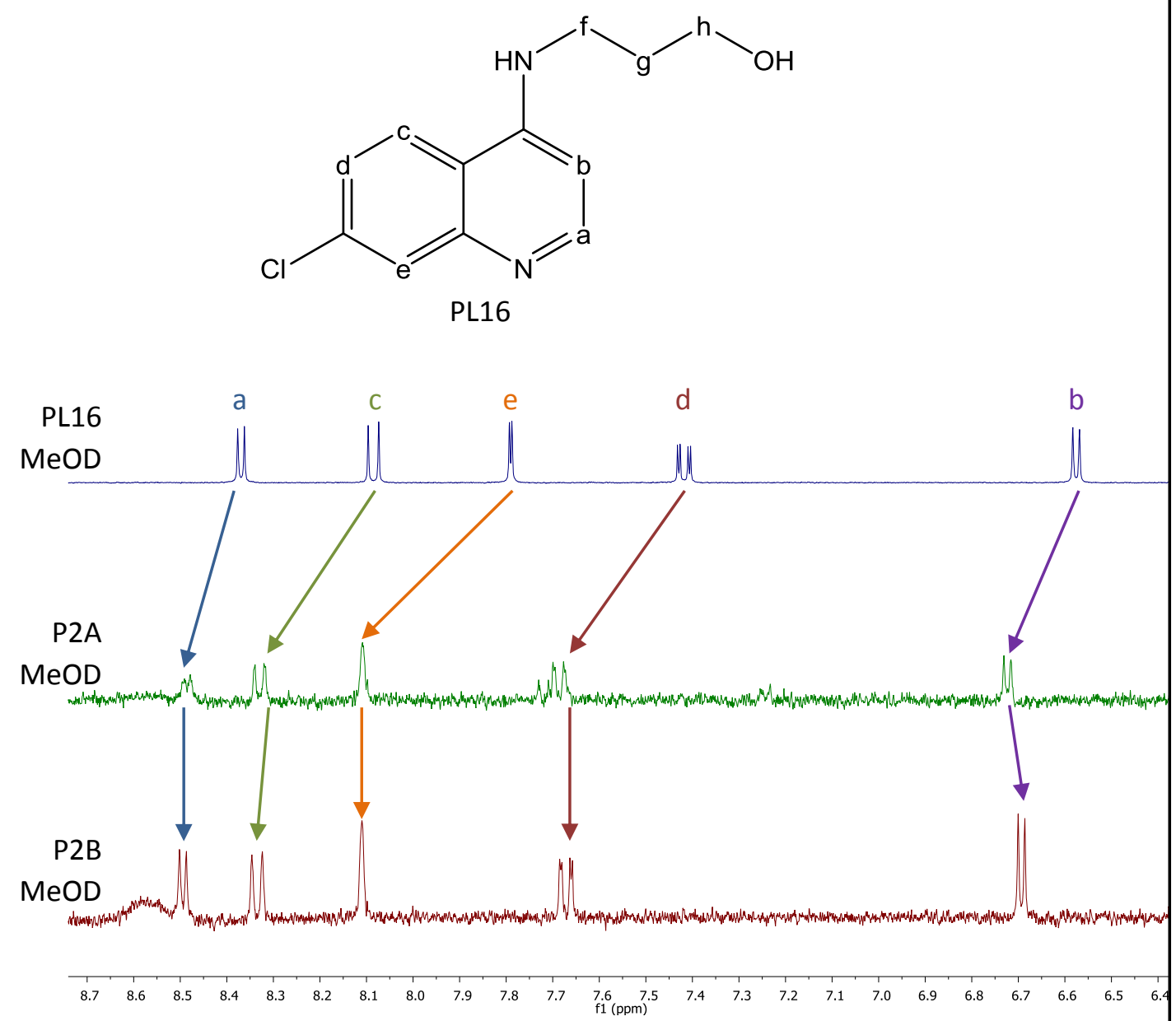

Figure 15: The aromatic regions of PL16, P2A, and P2B. Notice the similar chemical shifts. The structure of PL16 is above the spectra with labeled hydrogens. 


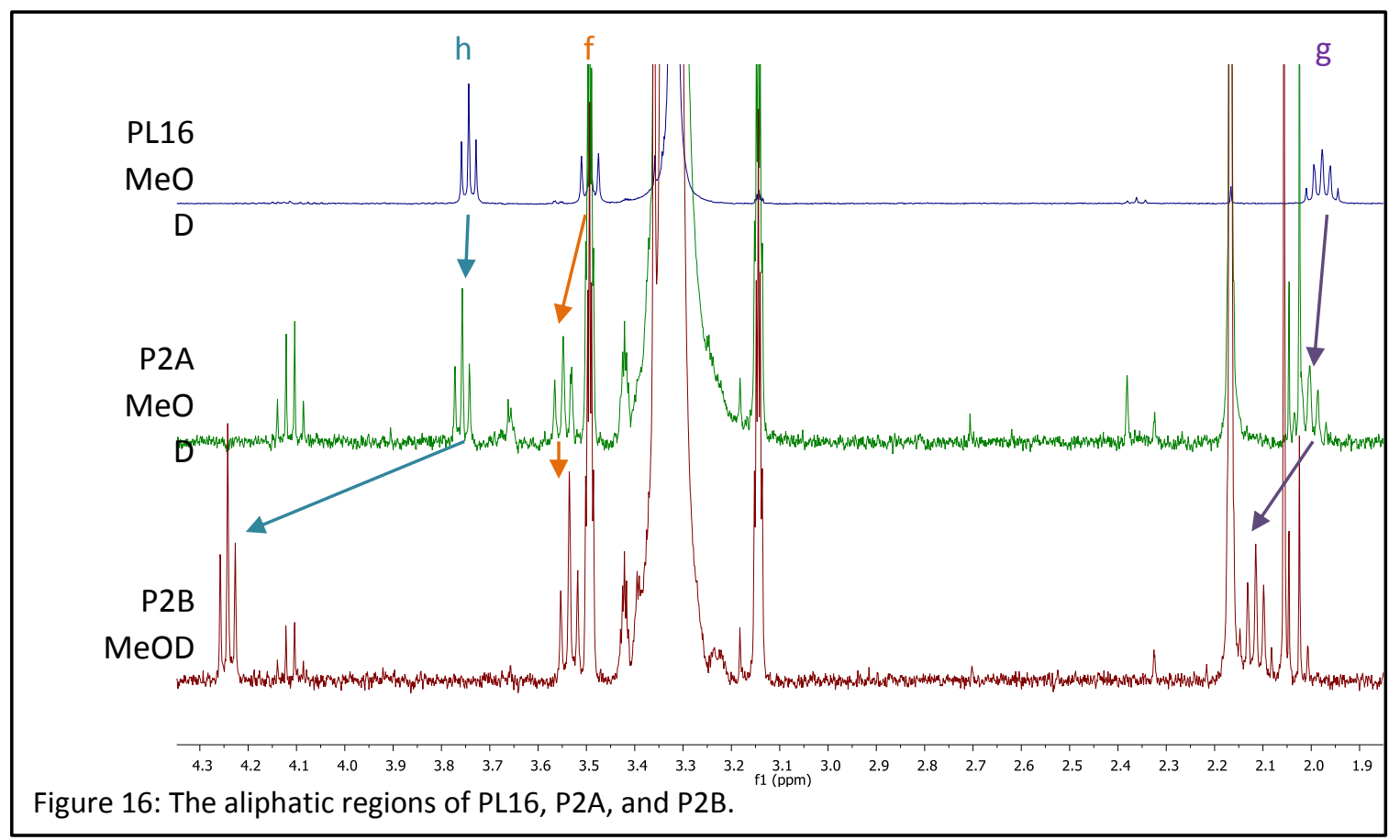




\section{Product 3}

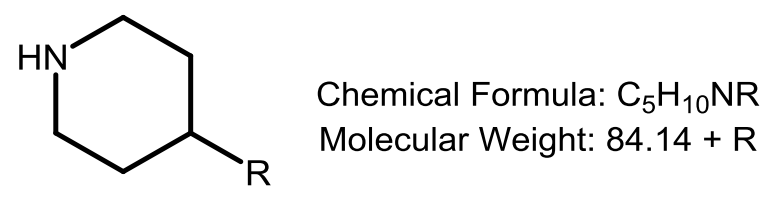

Figure 17: Proposed structure of product 3 (P3).

Product 3 (P3) was determined to consist of a piperidine, but the exact structure is not known. The ${ }^{1} \mathrm{H}$ NMR spectrum of $\mathrm{P} 3$ in $\mathrm{D}_{2} \mathrm{O}$ and $\mathrm{MeOD}$ is shown in the bottom two spectra of Figure 18 and the top two spectra are PL69 in $\mathrm{CDCl}_{3}$ and MeOD. The piperidine peaks of PL69 are indicated in the structure of PL69 and the ${ }^{1} \mathrm{H}$ NMR peaks of those hydrogens are labeled on spectra. The relationships of the piperidine peaks of PL69 to the peaks of P3 are indicated by arrows. After obtaining spectra for $\mathrm{P} 3$ in $\mathrm{D}_{2} \mathrm{O}$ and $\mathrm{MeOD}$, the four peaks of the hydrogens in the 2- and 3-positions were easily identified. $\mathrm{A}^{1} \mathrm{H}$ NMR peak for the single hydrogen in the 4-position has not yet been identified for P3. During one of the many rotoevaporations and dryings to remove extra solvent, P3 was lost from the round bottom flask in which it was stored. No further NMR analyses were able to be done in P3 besides ${ }^{1} \mathrm{H}$ NMR spectroscopy and COSY, and, unfortunately, a mass spectrum was not obtained for this product.

In order to attempt to further elucidate the structure of P3 after having lost the product, the ${ }^{1} \mathrm{H}$ NMR spectrum of $\mathrm{P} 3$ was compared with two substituted piperidines, 4hydroxypiperidine and 4-aminopiperidine (Figure 19). The ${ }^{1} \mathrm{H}$ NMR spectra of these two piperidines didn't match the spectra obtained for P3. The splitting observed in the three compounds were similar, but the chemical shifts were very different. Both 4- 
aminopiperidine and 4-hydroxypiperidine had well resolved peaks for the hydrogen at the 4 position, which is not present in the spectra for P3. The peaks had much different shifts as well. For the time being, the complete structure of $\mathrm{P} 3$ will remain unknown, though, it is probable there is a piperidine group.

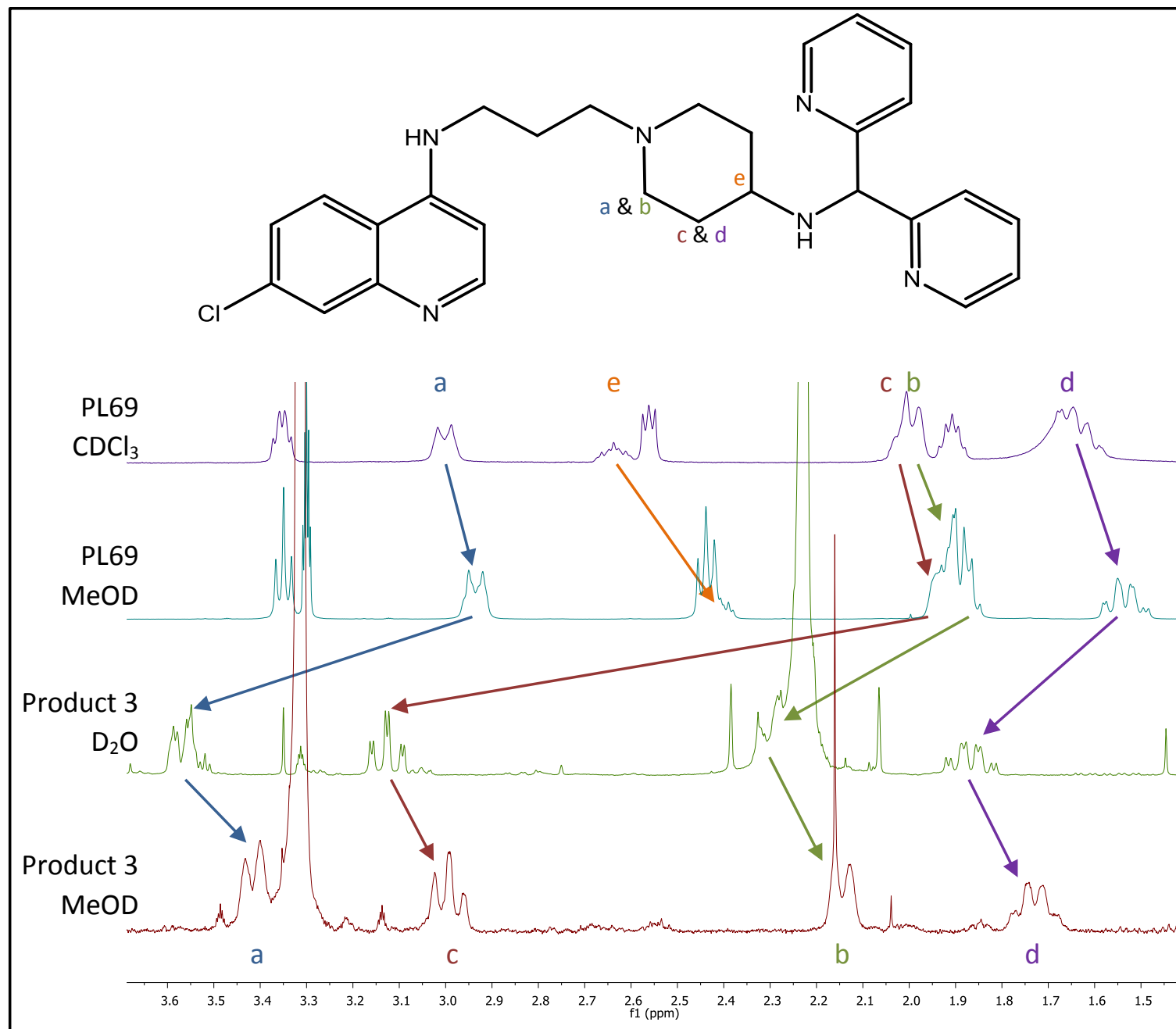

Figure 18: The structure of PL69 with the piperidine assignments. The spectra shows the ${ }^{1} \mathrm{H}$ NMRs of PL69 and P3 in different solvents as well as assignments of the peaks with respect to PL69. 


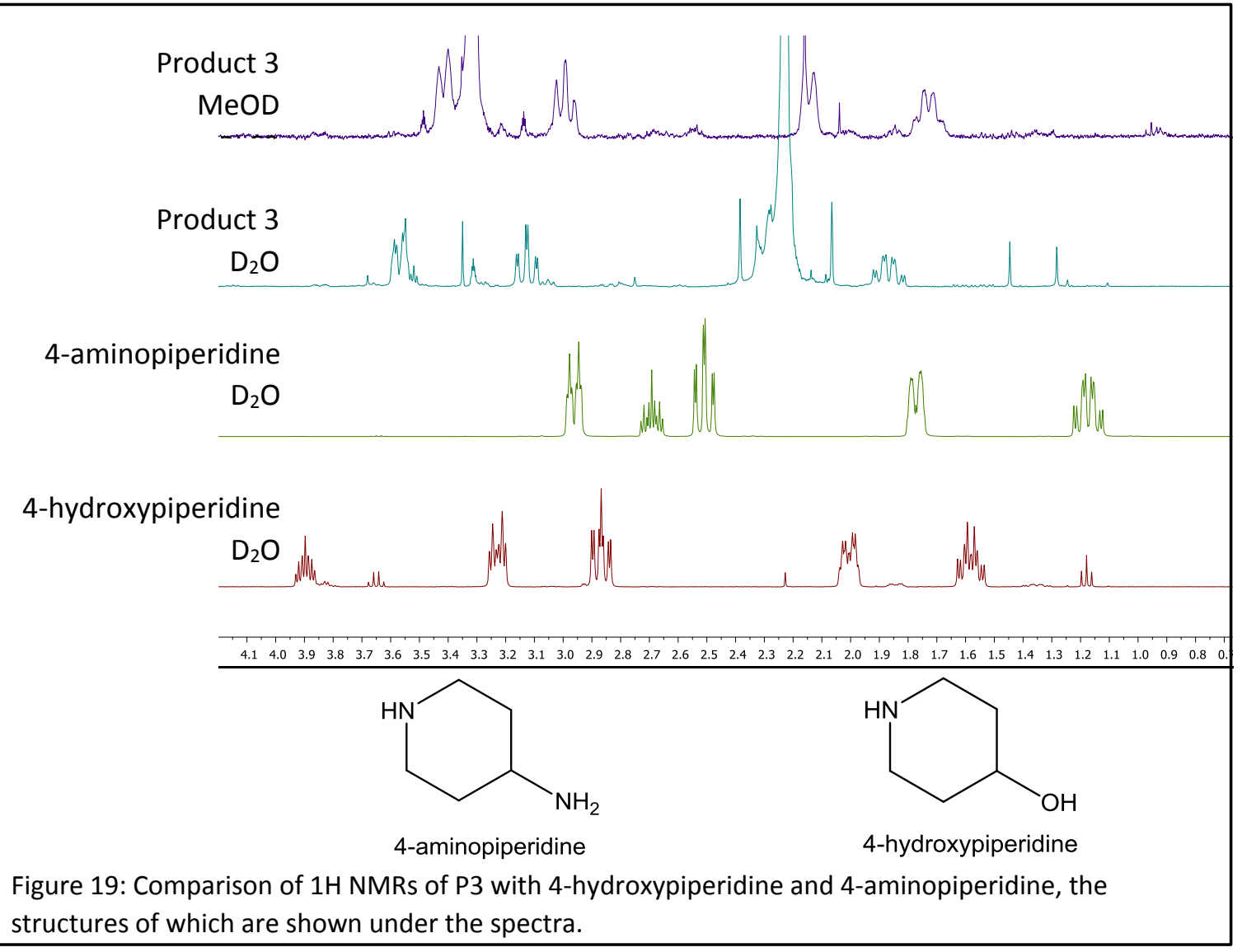

\begin{tabular}{|c|c|c|c|}
\hline Position & $\begin{array}{c}\text { Observed } \\
\text { shift (ppm) }\end{array}$ & Integration & Multiplicity \\
\hline $\mathrm{a}$ & 3.57 & 1 & $\mathrm{dd}$ \\
\hline $\mathrm{b}$ & 2.30 & 1 & $\mathrm{dd}$ \\
\hline $\mathrm{c}$ & 3.13 & 1 & $\mathrm{td}$ \\
\hline $\mathrm{d}$ & 1.87 & 1 & $\mathrm{qd}$ \\
\hline $\mathrm{e}$ & $3.52 ?$ & $?$ & $?$ \\
\hline
\end{tabular}

Figure 20: Structure of $\mathrm{P} 3$ with letter assignments correlating to the ${ }^{1} \mathrm{H}$ NMR shifts in the table. 


\section{Product 4}

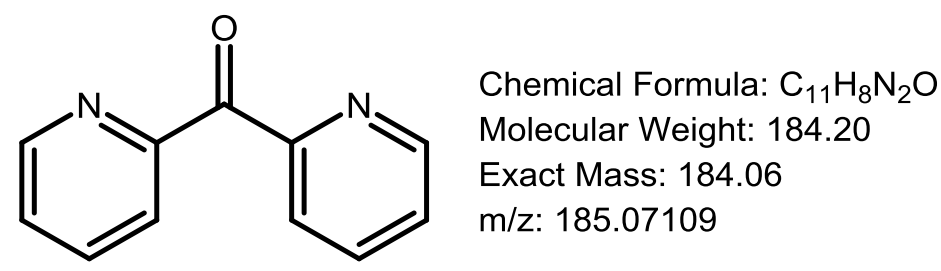

Figure 21: Proposed structure of product 4 (P4).

Product 4 (P4) was present in all of the PL69 incubations with Fe(III)TPPS and was present in large enough quantities to make it easy to collect ${ }^{1} \mathrm{H}$ NMR data with good signal to noise ratio $(S / N)$. Upon collecting this product, running a ${ }^{1} \mathrm{H} N M R$ spectrum, and comparing the ${ }^{1} \mathrm{H}$ NMR spectrum of PL69, it was thought that P4 was probably some sort of pyridine. However, the chemical shifts of this product were very different than the shifts of the pyridine in PL69; some of the peaks were shifted farther downfield than they had been in PL69 (Figure 22). The hydrogen at the 2-position of the pyridine shifted from $7.51 \mathrm{ppm}$ in PL69 to $8.11 \mathrm{ppm}$ inP4, indicating that a more electronegative group had been added near that hydrogen. It was originally thought that this product was picolinic acid, which is pyridine with a carboxyl group attached at the 2-position.

Mass spectrometry data was obtained for P4 giving a mass $(\mathrm{m} / \mathrm{z}=185.07109)$ that was much larger than what would be expected for picolinic acid (exact mass $=123.03203$ amu). To get an $\mathrm{m} / \mathrm{z}$ of $185.07109 \mathrm{amu}$, there needed to be two connected pyridine rings. Based on the mass, the chemical formula of $\mathrm{M}+1$ was determined to be $\mathrm{C}_{11} \mathrm{H}_{9} \mathrm{~N}_{2} \mathrm{O}$. The identity of P4 was postulated to be di(2-pyridyl) ketone. The ppm differences obtained from the P4 are very low and within a reasonable difference (Table 7). 
The carbonyl group attaching the two pyridyl rings caused the hydrogen at the 2position to shift drastically downfield and also shifted the other hydrogens slightly farther downfield. $A^{1} \mathrm{H}$ NMR spectrum obtained for known di(2-pyridyl) ketone in chloroform was compared to a sample of P4 in chloroform to ensure the structure was elucidated correctly. Those ${ }^{1} \mathrm{H}$ NMR spectra are shown in Figure 23 and listed in Figure 24. With the combination of ${ }^{1} \mathrm{H}$ NMR spectra, mass spec, and comparison to a known structure, the identity of P4 was determined to be di(2-pyridyl) ketone.

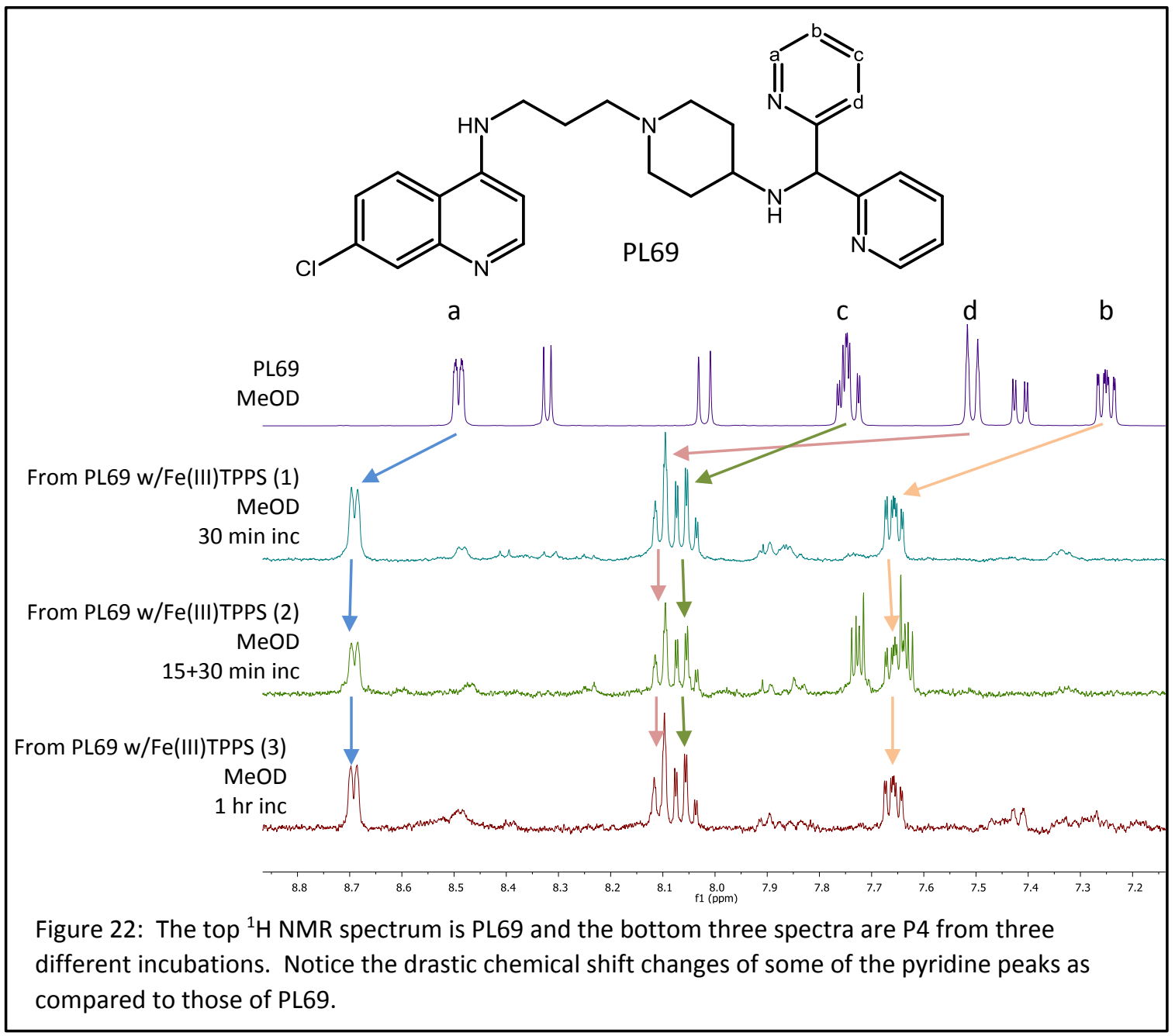




\begin{tabular}{|c|c|c|}
\hline Source of Product & Obtained m/z & $\Delta \mathrm{ppm}$ \\
\hline theoretical $\mathrm{C}_{11} \mathrm{H}_{9} \mathrm{~N}_{2} \mathrm{O}$ & 185.07149 & $\mathrm{n} / \mathrm{a}$ \\
\hline $\mathrm{P} 4[\mathrm{Fe}(\mathrm{III})$ TPPS (30min)] & 185.07133 & 0.86453 \\
\hline $\mathrm{P} 4[\mathrm{Fe}(\mathrm{III}) \mathrm{TPPS}(15+30 \mathrm{~min})]$ & 185.07028 & 6.4861 \\
\hline P4 [Fe(III)TPPS (1hr)] & 185.07108 & 2.1916 \\
\hline
\end{tabular}

Table 7: Mass spectra data obtained for $\mathrm{P} 4$ from three different incubations. The top row, $\mathrm{C}_{11} \mathrm{H}_{9} \mathrm{~N}_{2} \mathrm{O}$, is the theoretical exact mass of $\mathrm{P} 4+\mathrm{H}$, which would be seen in the positive mode mass spectrum.

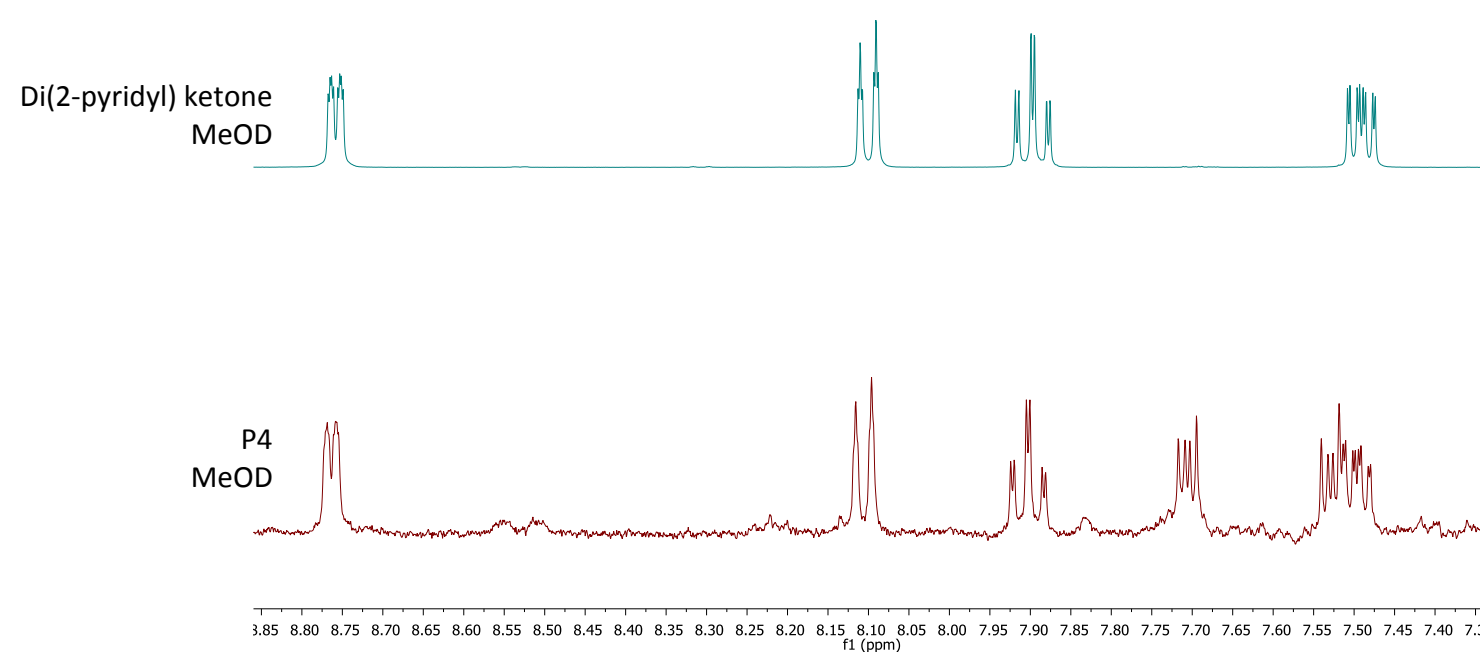

Figure 23: The top ${ }^{1} \mathrm{H}$ NMR spectrum is di(2-pyridyl) ketone and the bottom spectrum is P4 obtained from the 30 minute PL69 incubation with Fe(III)TPPS. The chemical shifts are basically identical, indicating the identity of P4. There are two impurity peaks at 7.53 and $7.71 \mathrm{ppm}$. The identity has not been elucidated, but they are present in other sample spectra so they were determined to be impurities.

\begin{tabular}{|c|c|c|c|c|}
\hline Position & $\begin{array}{c}\text { Observed } \\
\text { shift (ppm) }\end{array}$ & $\begin{array}{c}\text { Theoretical } \\
\text { shift (ppm) }\end{array}$ & Integration & Multiplicity \\
\hline $\mathrm{a}$ & 8.77 & 8.76 & 1 & $\mathrm{dt}$ \\
\hline $\mathrm{b}$ & 7.50 & 7.49 & 1 & $\mathrm{ddd}$ \\
\hline $\mathrm{c}$ & 7.91 & 7.90 & 1 & $\mathrm{ddd}$ \\
\hline $\mathrm{d}$ & 8.11 & 8.10 & 1 & $\mathrm{dt}$ \\
\hline
\end{tabular}

Figure 24: The chemical shifts of P4 (observed shift) as compared with di(2-pyridyl) ketone (theoretical shift). The integration and multiplicity of the peaks are also listed and correlate with those of di(2-pyridyl) ketone. 


\section{$\underline{\text { Product } 5}$}

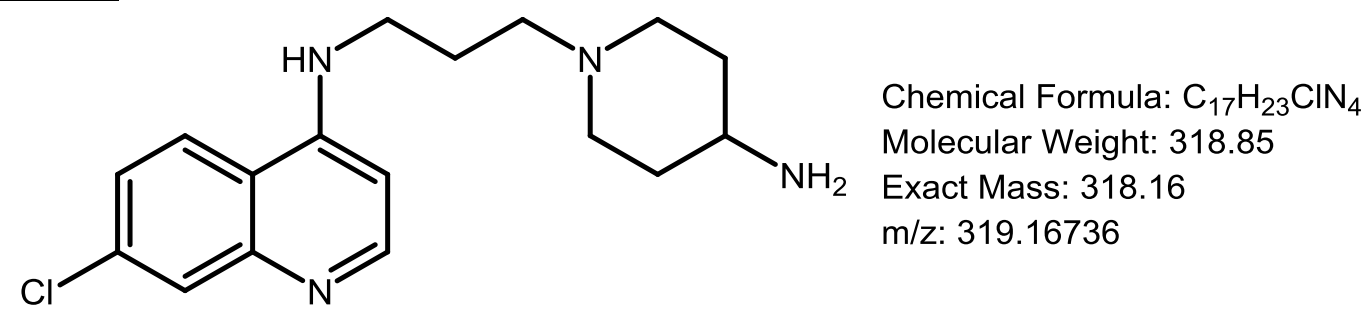

Figure 25: Proposed structure of product 5 (P5).

Product 5 (P5) is similar to M9 seen in the incubations done by WuXi AppTec, except M9 has a hydroxyl at the 4-position of the piperidine rather than an amine. This product was not isolated during the initial porphyrin incubations, but was isolated in two of the more recent porphyrin incubations. The ${ }^{1} \mathrm{H}$ NMR spectrum of $\mathrm{P} 5$ is similar to that seen for PL69, except the pyridyl peaks were missing from the aromatic region (Figure 26 and 27). The mass spectrum (Figure 28) obtained for P5 had an $M+1$ of 319.16736 amu, with a difference of only 5 ppm from the theoretical mass of $\mathrm{C}_{17} \mathrm{H}_{24} \mathrm{~N}_{4} \mathrm{Cl}$ (319.16895 amu). Based on the presence of the same peaks in the aromatic and aliphatic region as PL69 as well as factoring in the mass, the P5 was determined to be $\mathrm{N}$-(3-(4-aminopiperidin-1yl)propyl)-7-chloroquinolin-4-amine. 

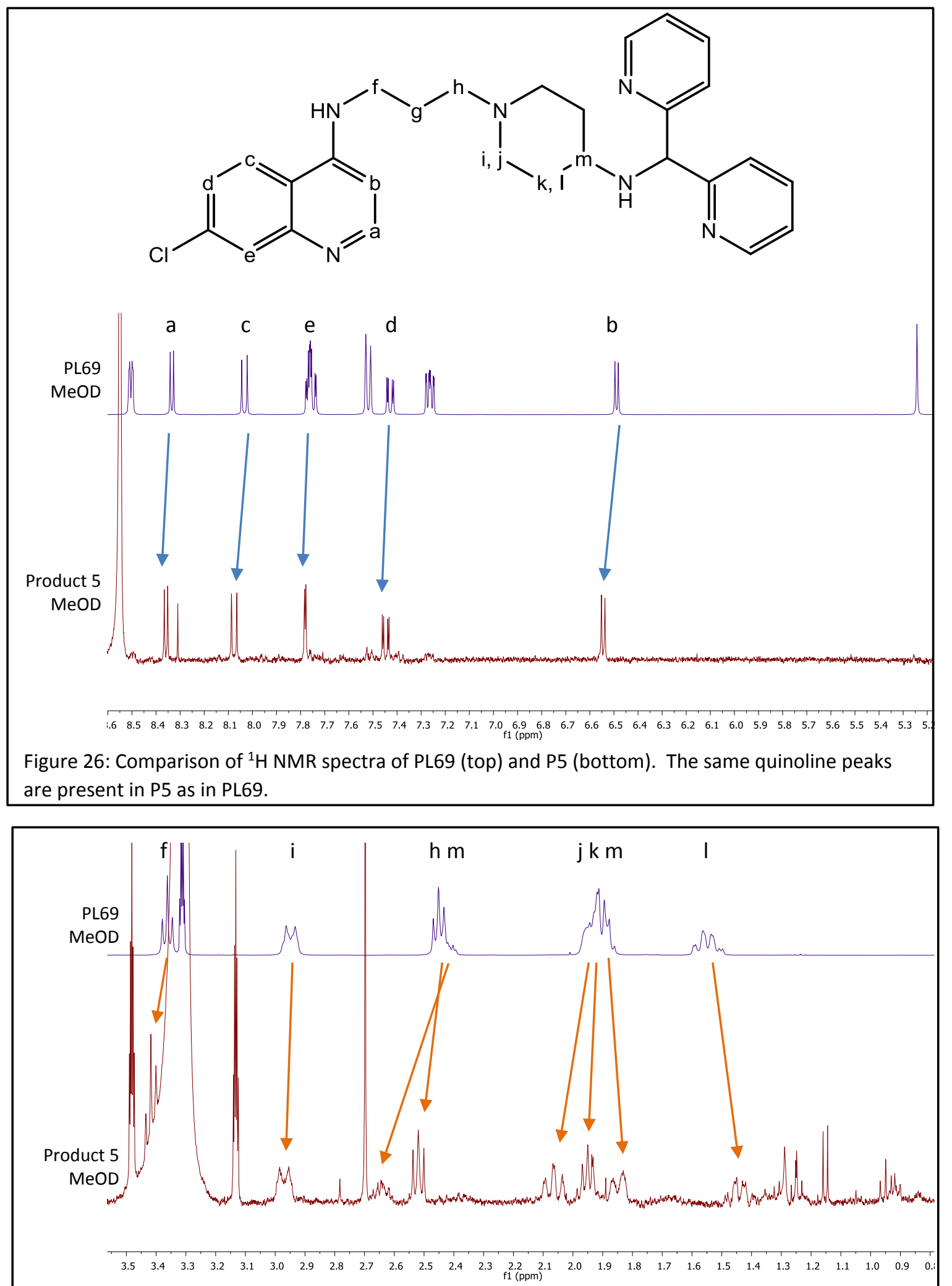

Figure 27: Comparison of the aliphatic region of PL69 (top) and P5 (bottom). The same peaks are present in P5, but slightly shifted compared to PL69. 


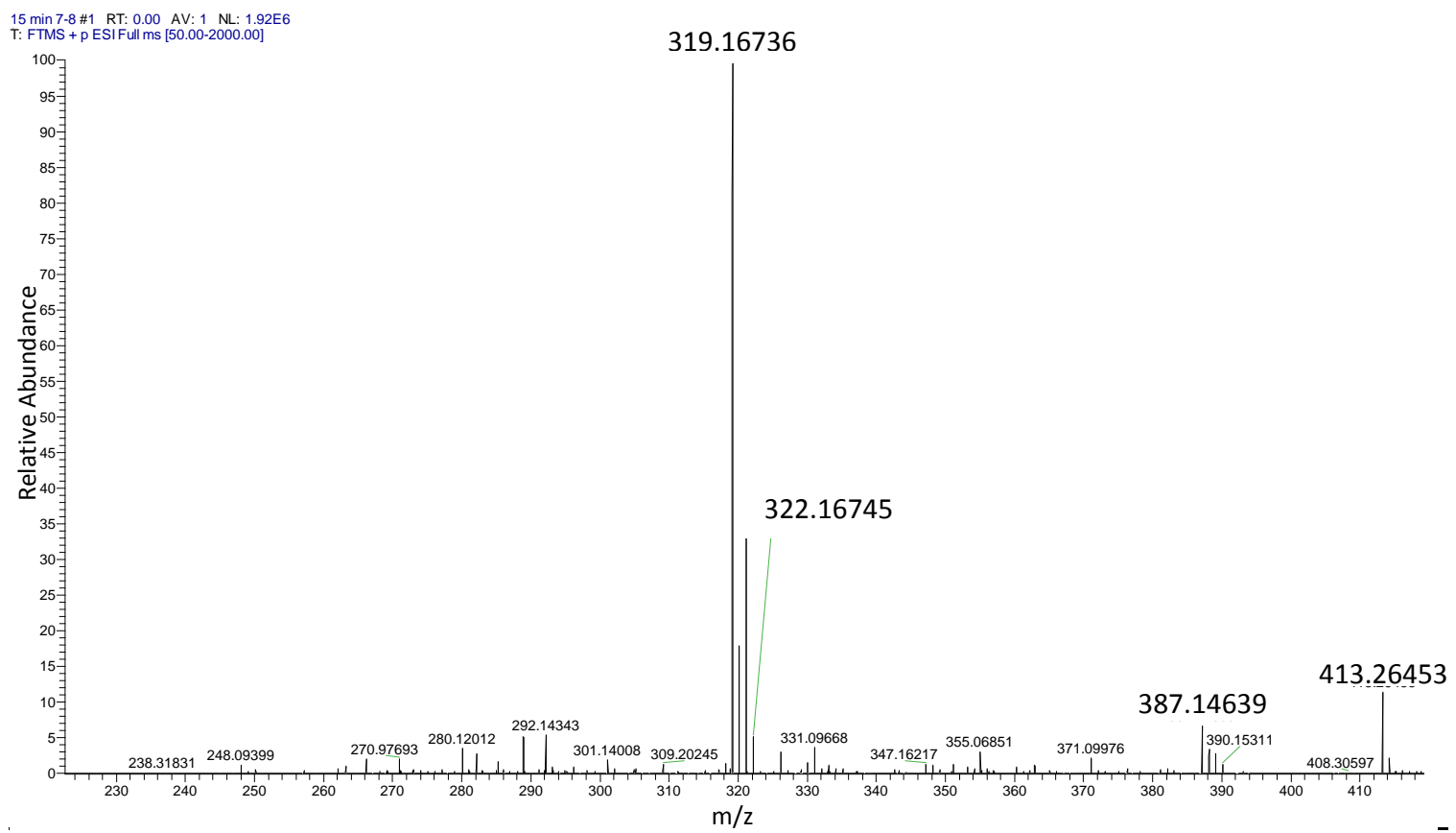

Figure 28: Mass spectrum of P5. 


\section{Product 6}

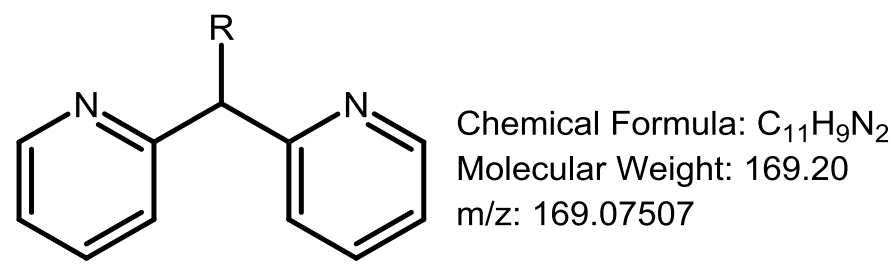

Figure 29: Proposed structure of product 6 (P6).

Product 6 (P6) proved to be a tricky compound to elucidate. Proton NMR spectra as well mass spectra have been obtained for P6, but the puzzle of the complete structure has not been determined. The ${ }^{1} \mathrm{H}$ NMR spectrum for this product in MeOD gave five peaks in the aromatic region (Figure 30 ) and no other peaks. By looking at the chemical shifts of P6 in comparison to PL69, P6 was determined to contain at least one pyridine ring. To try to see any exchanging protons, P6 was run on the NMR in deuterated dimethyl sulfoxide (DMSO-d6), which gave very broad peaks. Sodium deuteroxide (NaOD) was added to the DMSO-d6 sample to try to get sharper peaks, even though adding $\mathrm{NaOD}$ introduced water to the sample, removing the exchanging proton peaks. Adding NaOD sharpened up the peaks quite a bit, but also started to oxidize the sample. Every ${ }^{1} \mathrm{H}$ NMR spectra obtained after adding NaOD made the P6 peaks smaller and some other peaks larger, including peaks corresponding to P4, di(2pyridyl) ketone (Figure 30). There seems to be oxidation of $\mathrm{P} 6$ to other products besides just P4, as well.

A mass spectrum was obtained for $\mathrm{P} 6$ which gave an $\mathrm{m} / \mathrm{z}$ in the positive mode of 169.07507 amu, corresponding to a chemical formula of $\mathrm{C}_{11} \mathrm{H}_{9} \mathrm{~N}_{2}$ (Figure 32). The ppm 
difference between the $\mathrm{m} / \mathrm{z}$ and the exact mass of $\mathrm{C}_{11} \mathrm{H}_{9} \mathrm{~N}_{2}$ is $9 \mathrm{ppm}$. This formula corresponds to the actual $\mathrm{m} / \mathrm{z}$, rather than the $M+1$, because there was not a structure that could be identified, that made sense, for a formula of $\mathrm{C}_{11} \mathrm{H}_{8} \mathrm{~N}_{2}$. The $\mathrm{m} / \mathrm{z}$ obtained from the mass spectrum led to the belief that P6 has two pyridine rings. Multiple structures were examined in order to try to get a product with just one pyridine ring, but those structures always added in more hydrogens that weren't present in the ${ }^{1} \mathrm{H}$ NMR spectrum. It is probable that the molecule fragmented upon entering the mass spectrometer giving a structure shown in Figure 31. This structure fits with the mass obtained, but is most likely just a fragment of the actual molecule of P6. One explanation could be a hydroxyl group attached to the carbon linking the two pyridyl rings which broke off upon entering the MS. Adding NaOD to the NMR sample would have neutralized the acid and added hydroxide ions that could oxidize the molecule.

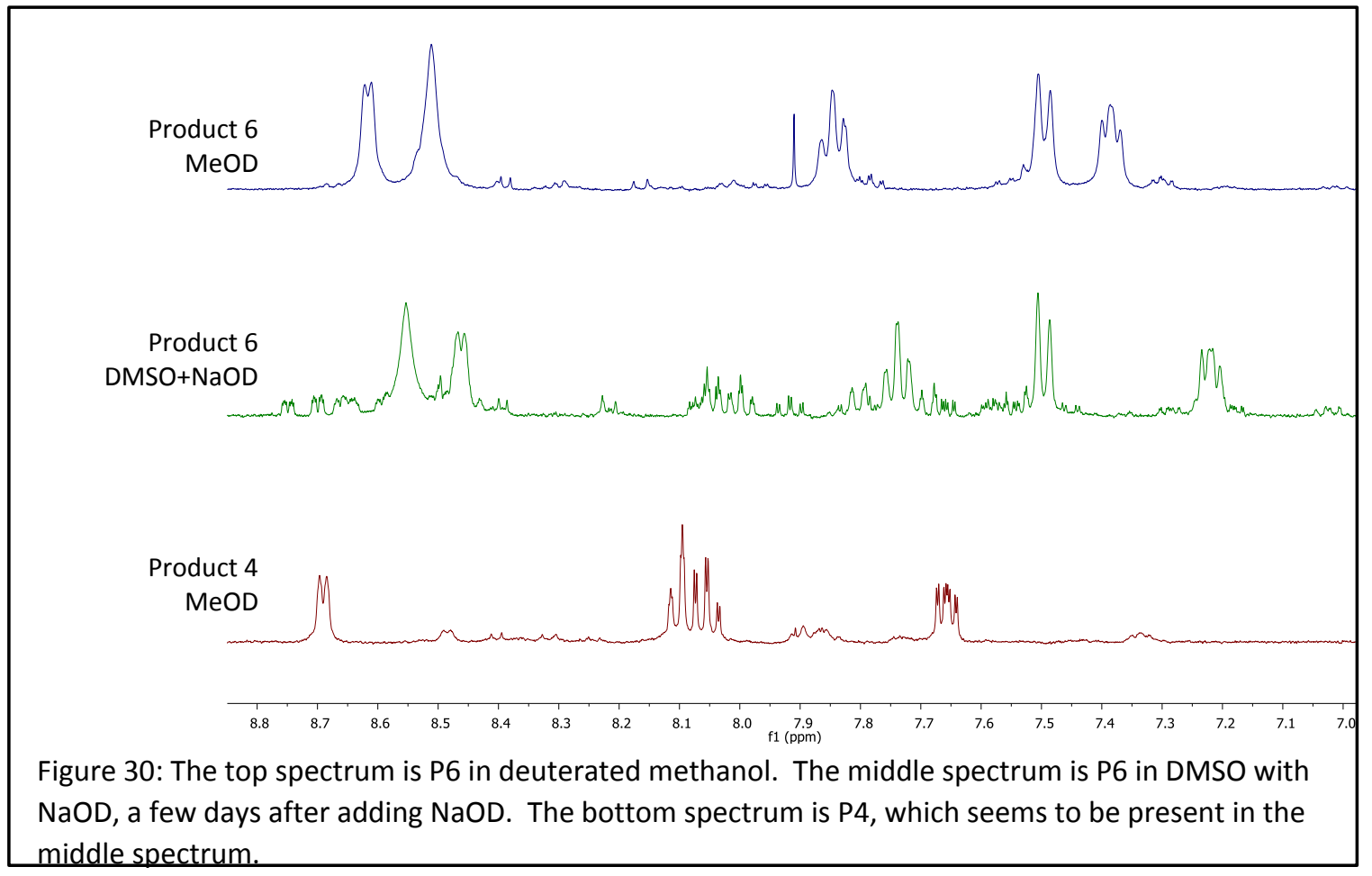




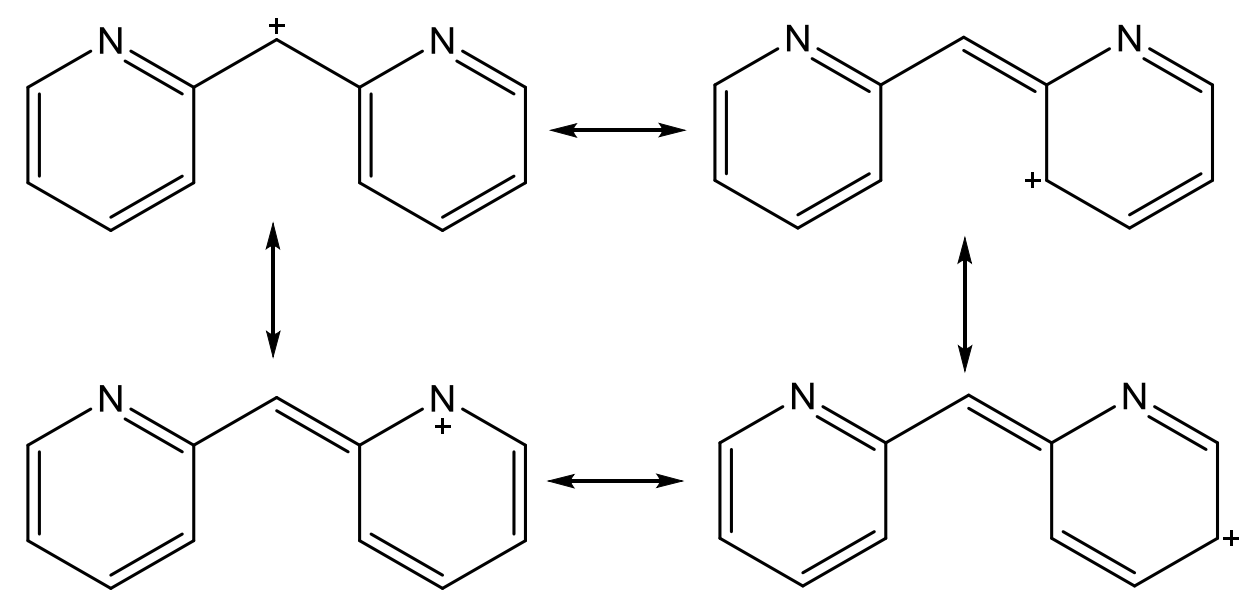

Figure 31: Possible explanation of P6 fragment measured by the MS. The positive charge could be stabilized by the aromatic rings.

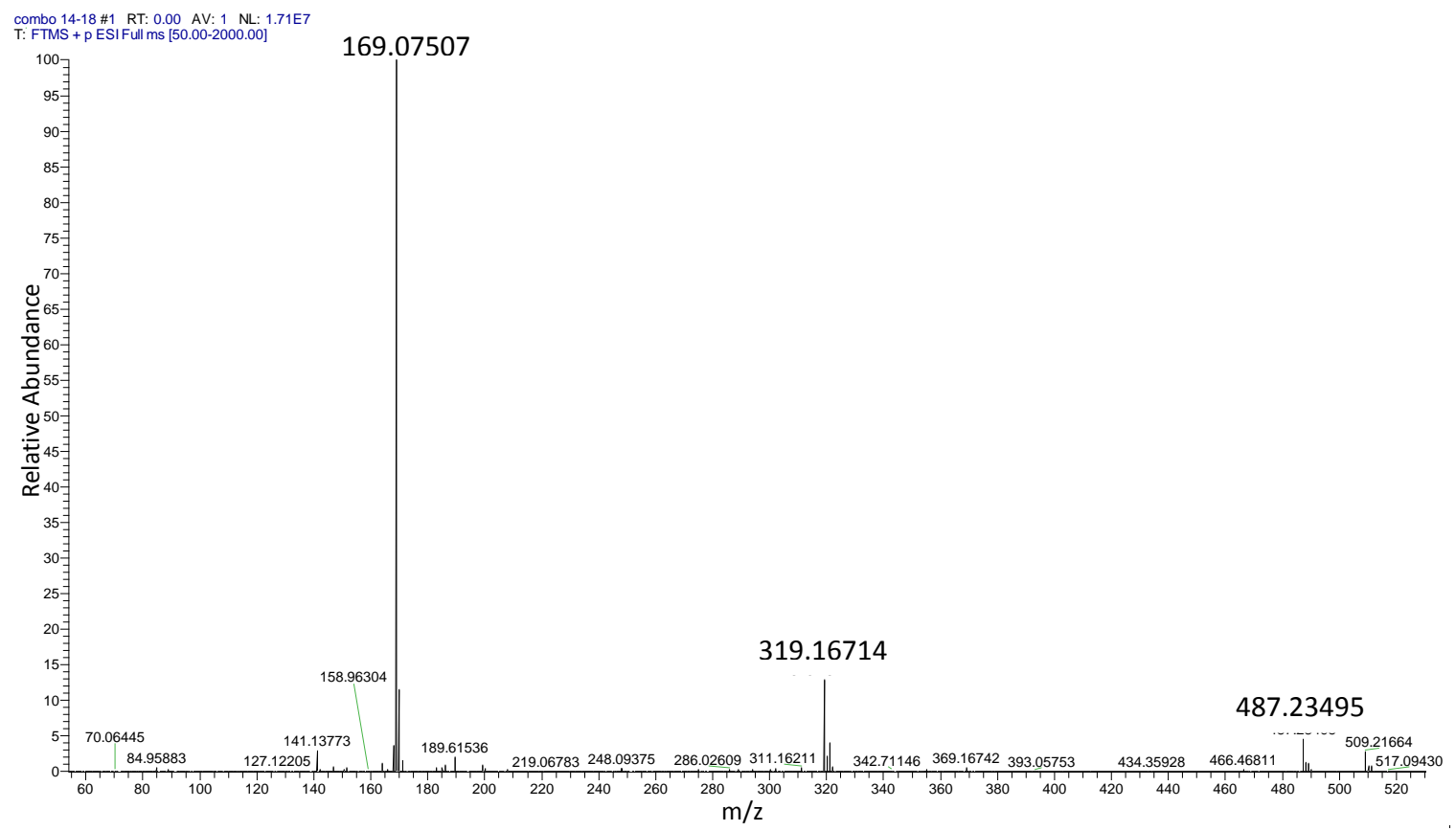

Figure 32: Mass spectrum of P6 


\section{Product 7}

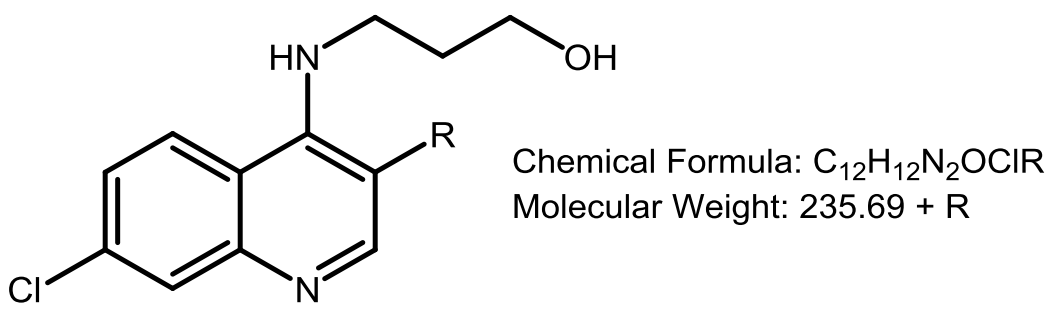

Figure 33: Proposed structure of product 7 (P7).

The full identity of product 7 (P7) has not been fully elucidated, but the pieces of this product are known. The ${ }^{1} \mathrm{H}$ NMR spectrum of $\mathrm{P} 7$ has four peaks in the aromatic region (Figure 34). The three hydrogens on the ring with the chlorine have similar peaks as seen in the ${ }^{1} \mathrm{H}$ NMR spectrum of PL69. However, there is only one peak present in the spectrum to represent the hydrogens on the ring with the nitrogen. Sometimes, the hydrogen at the 2-position is not seen in the ${ }^{1} \mathrm{H}$ NMR spectrum, depending on solvent, but the hydrogen at the 3-position would still be seen as a doublet, so it can be assumed the 2-position hydrogen was present. However, in the case of this product, there is no hydrogen in the area of the hydrogen at the 3-position, but there was a singlet slightly up field from where the hydrogen at the 2-position usually is, leading to the belief that there was a substitution at the 3-position. The j-values calculated for the spectrum of P7 in the aromatic region were similar to those of PL16, which lead to the peak assignments. The aliphatic peaks of P7 correspond to those seen in the aliphatic region of PL16 (Figure 35). 
There were three peaks seen in the aliphatic region that correlated to the three carbon chain attached to the chloroquinoline ring. There didn't seem to be any other hydrogens attached to carbons present in the ${ }^{1} \mathrm{H}$ NMR spectrum. The identity of the " $\mathrm{R}$ " group at position three of the ring was not elucidated. The product was, unfortunately, lost before obtaining a mass spectrum.

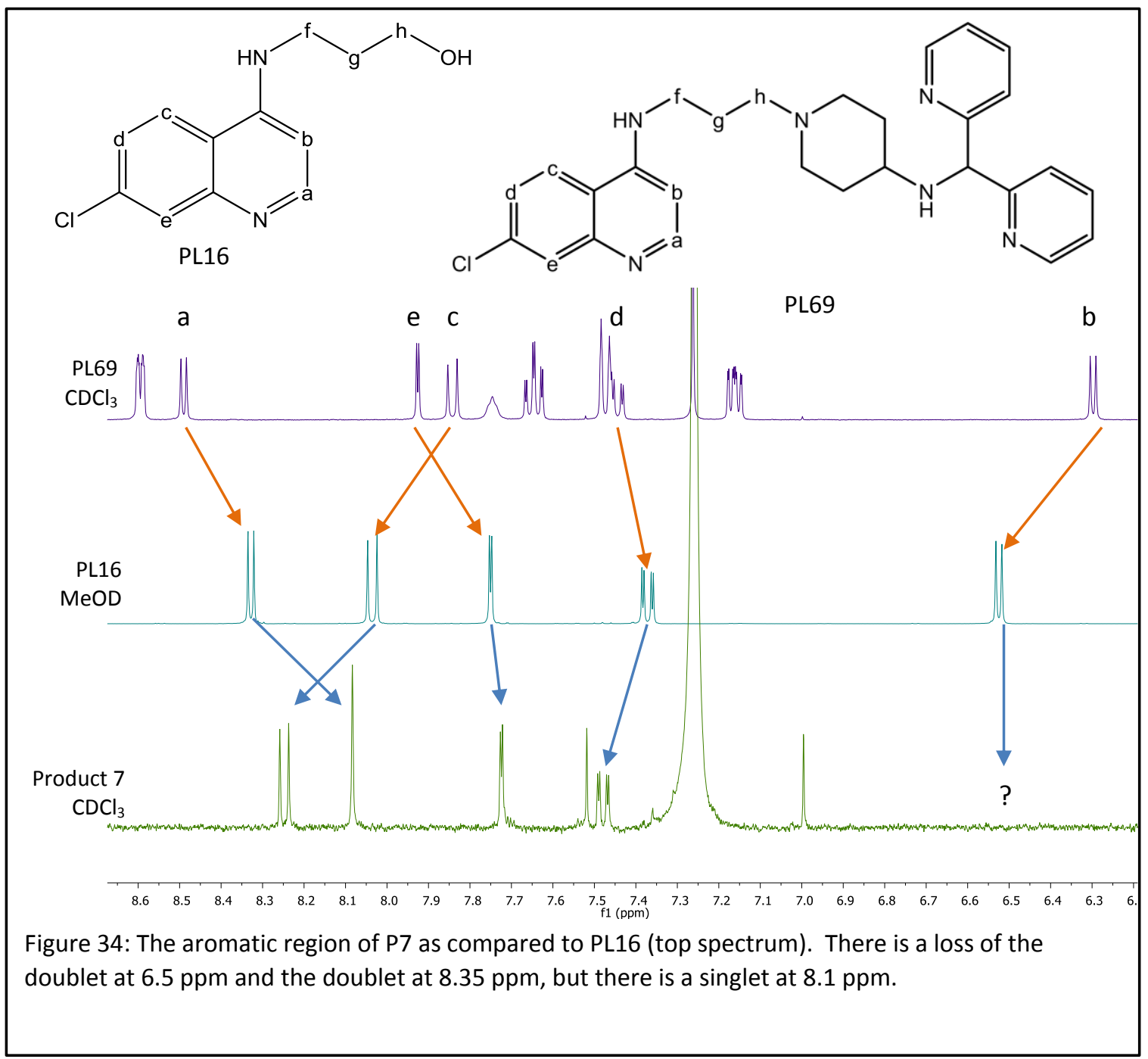




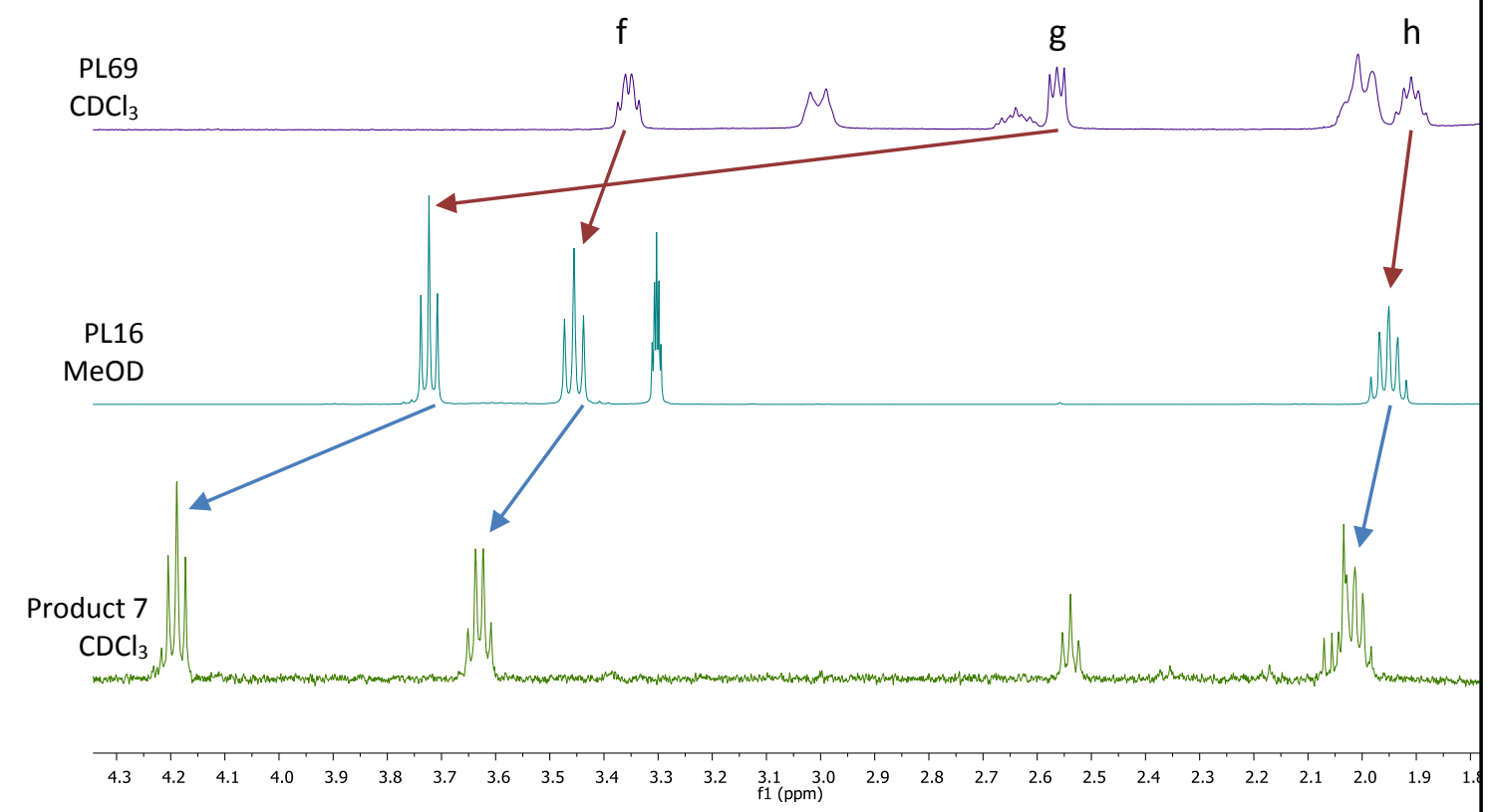

Figure 35: The aliphatic region of P7 as compared to PL16 (top spectrum). The P7 peak peak at 3.64 is a quartet because the solvent is $\mathrm{CDCl}_{3}$ and the hydrogens of that peak are seeing the $-\mathrm{NH}$ attached to the quinoline ring. The triplet at 2.54 is considered an impurity because it does not integrate with the other peaks. It could possibly be from another product in the sample. 


\section{Product 8}

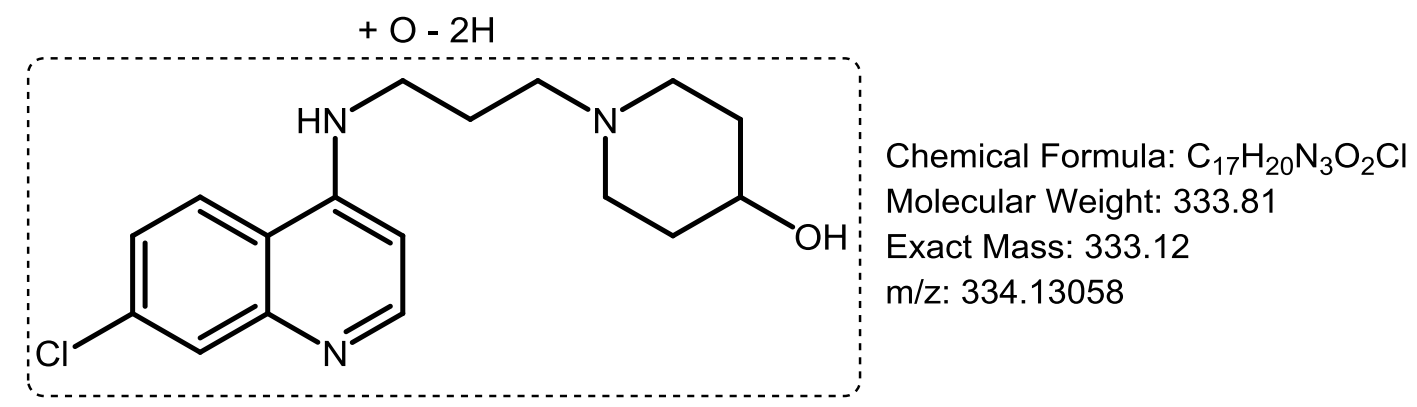

Figure 36: Proposed structure of product 8 (P8).

Product 8 (P8) was only analyzed by mass spectrometry, there was not enough to obtain a ${ }^{1} \mathrm{H}$ NMR spectrum. It was obtained in a pure form, but was present in the mass spectrum of some of the other products, including P5. The structures resembles M9 from the metabolism of PL69 completed by WuXi AppTech (Figure 7) with a carbonyl added in place of two hydrogens. The ppm difference between the $\mathrm{m} / \mathrm{z}$ and the $\mathrm{C}_{17} \mathrm{H}_{21} \mathrm{~N}_{3} \mathrm{O}_{2} \mathrm{Cl}(\mathrm{M}+1)$ is just $5 \mathrm{ppm}$, which means this formula is probably correct. The full structure cannot be known without NMR data, but the structure above is a probable structure based on the mass spec data. 


\section{Product 9}

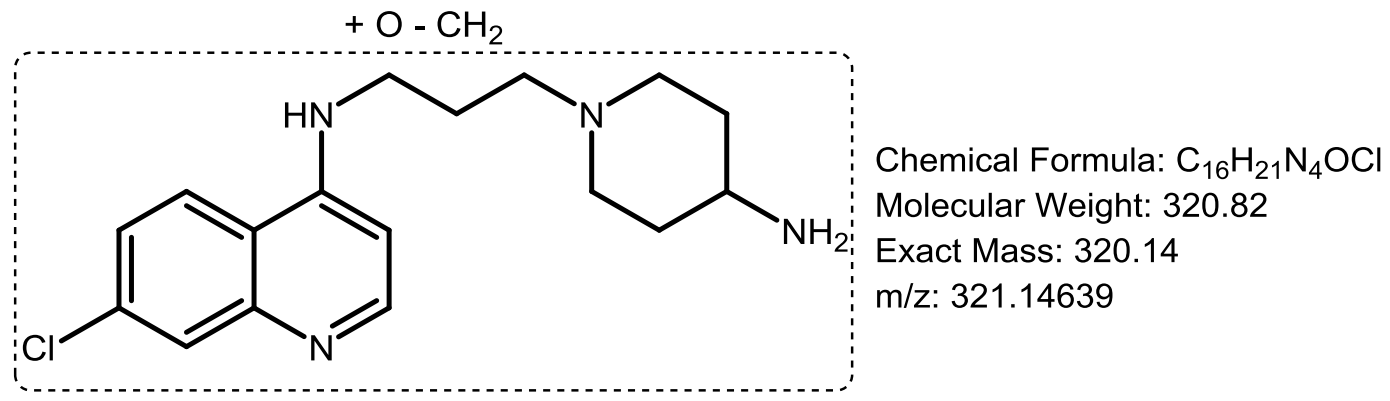

Figure 37: Proposed structure of product 9 (P9).

Product 9 (P9) has a theoretical structure similar to P5, except with an added carbonyl in place of a methylene group. There was not enough of this product to obtain a well resolved ${ }^{1} \mathrm{H}$ NMR spectrum, so it was only analyzed by MS. This type of structure would probably make the piperidine ring open and an aldehyde form, which is one of the few ways a methylene group can be removed without breaking the molecule. Another possible structure is formation of an ether at the 2- or 3-position of the piperidine. Possible structures are on the next page in Figure 38. The exact mass of $\mathrm{M}+1$ is $321.14821 \mathrm{amu}$, which is a ppm difference of $6 \mathrm{ppm}$ compared to the $\mathrm{m} / \mathrm{z}$ obtained, 321.14639 amu. 

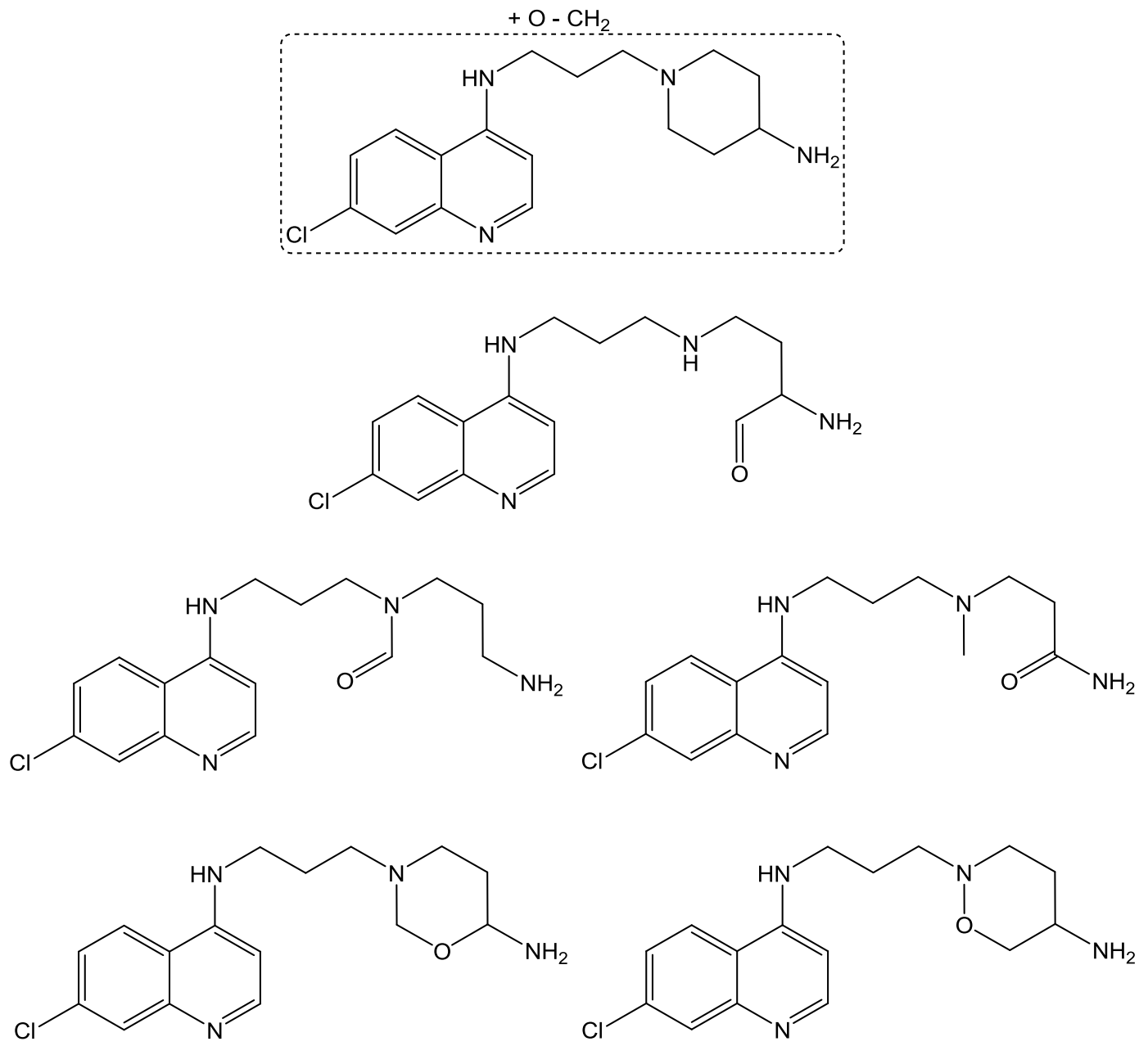

Figure 38: Possible structures for P9 to fit the chemical formula $\mathrm{C}_{11} \mathrm{H}_{21} \mathrm{~N}_{4} \mathrm{OCl}$. 


\section{$\underline{\text { Discussion }}$}

Oxidation of a reversed chloroquine, PL69, with Fe(III)TPPS allowed for analysis of oxidation products that are similar to the products obtained with in vitro metabolism methods. It was hypothesized the products of the porphyrin oxidations would be similar to those of in vitro methods, but the degree of similarity for PL69 was not known. The results discussed in this thesis show that, although some of the products of the porphyrin oxidations were slightly different than those found in the metabolism with microsomes, they provided a reasonable starting point for metabolism analysis. The most prominent reaction that occurred was $\mathrm{N}$-dealkylation, but hydroxylation and oxidation were also seen.

Looking at the metabolite structures proposed by WuXi AppTec, comparisons can be made between the structures determined with Fe(III)TPPS and the structures determined with the rat, monkey, and human liver microsomes. Shown in Figure 40 (at the end of this section) are the structures of the metabolites formed by microsomes (red), as determined by WuXi AppTec, and the structures determined from the porphyrin oxidations (blue). When comparing side by side, many similarities are seen in the products of the microsome and porphyrin incubations. The microsomal incubations by WuXi AppTech included more dealkylations with addition of oxygen (hydroxyl or carbonyl), than what was seen with the porphyrin oxidations. This could possibly be due to higher oxidizing capabilities of CYP450s. P1 and P5 were all formed in the porphyrin 
incubations, as well as the microsomal incubations by WuXi AppTech. This indicates that the porphyrin method provides products that are similar, if not the same, as what would be expected in microsomal incubations.

Many oxidation products were produced from reacting PL69 with microsomes as well as with the iron porphyrin and hydrogen peroxide. Difficulty was encountered when trying to separate and analyze all of the products from one another, but the main products obtained were analyzed by NMR spectroscopy and/or mass spectrometry. A total of ten products were analyzed from incubations with PL69 and PL16 (a known metabolite of PL69). Unfortunately, some of the products were only analyzed by one method, either because they were produced in a small amount or the product was lost. Even though not all of the products were fully elucidated, the suggested structures provide a good framework for examining oxidation processes of other compounds with a porphyrin incubation.

There are multiple options for the next steps. First, another microsomal incubation could be done with PL69 now that there is a better method for separation of the compounds, in order to compare the products from both methods. Another option is to use the porphyrin system to examine the oxidation products of other reversed chloroquines. This same porphyrin system has been used to study one other compound from the Peyton lab, PL241, by Tess Harris (Figure 39). PL241 has a 4-amino-7chloroquinoline group on both sides of the molecule and produced only four oxidation products, making it much easier to study than PL69. The products seen in oxidation of 
PL241 followed the same patterns as seen for PL69, with the most prominent reaction being $N$-dealkylation. Once the identity of the in vitro or in vivo metabolites are fully known, the next step would be to examine toxicity as well as efficacy of each of the metabolites. This would probably include synthesizing the prominent metabolites and then testing them for activity and toxicity.

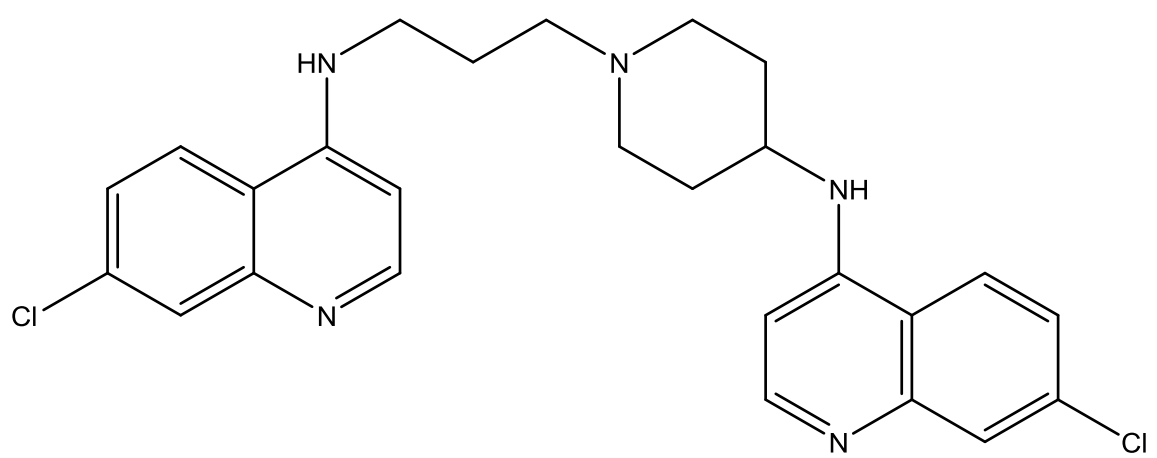

Figure 39: Structure of PL241.

Since the porphyrin system produced similar products as what was predicted in the microsomal incubations, this system could be used to develop an understanding of possible metabolites for multiple compounds before investing in the more expensive microsomal incubations. The porphyrin method at least could provide a framework for understanding what type of metabolism might occur in vivo. The process of separation and data collection could be improved to provide a much quicker, more efficient method to examine the products. Overall, using Fe(III)TPPS seemed to be a suitable mimic of CYP450 in that it produced similar products to those seen with CYP450. This method should be examined with other reversed chloroquines to determine the accuracy of oxidation as compared to microsomes and other sources of CYP450. 

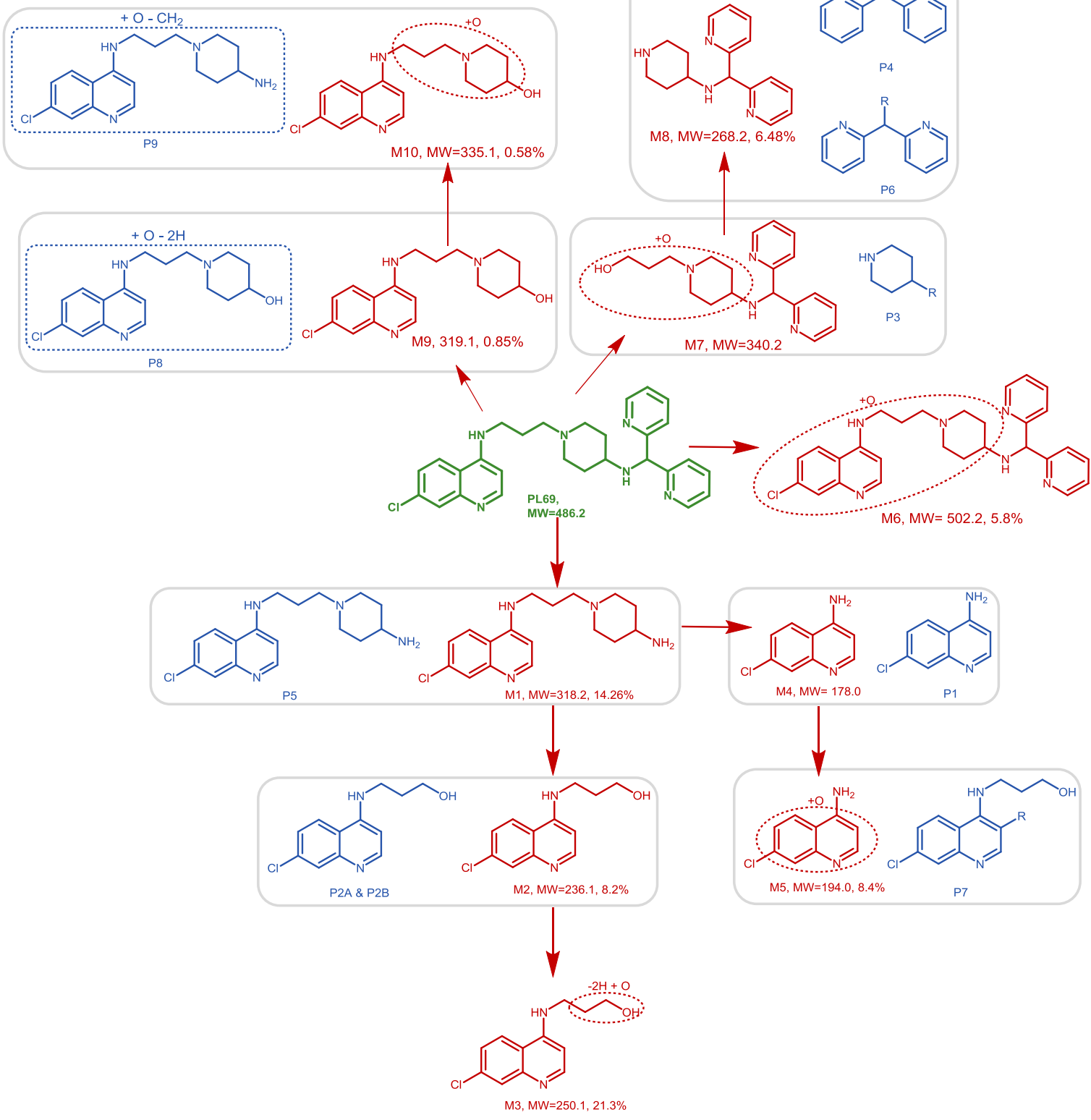

Figure 40: Comparison of the metabolites of PL69 determined by WuXi AppTech (red) with the products from this research that were elucidated by mimicking metabolism with microsomes and a metalloporphyrin system (blue). The oxidation products in blue are similar to the metabolites in red. This shows that using a metalloporphyrin system can provide a framework for understanding metabolism of a drug.

52 


\section{$\underline{\text { References }}$}

"10 Facts on Malaria." WHO. Mar. 2014. Web. 18 May 2014.

Andrews, Simeon, Steven J. Burgess, Deborah Skaalrud, Jane Xu Kelly, David H. Peyton. "Reversal Agent and Linker Variants of Reversed Chloroquines: Activities against Plasmodium Falciparum." Journal of Medicinal Chemistry 53 (2010): 916-19.

Baranczewski, Pawel, Andrzej Stanczak, Kathrin Sundberg, Richard Svensson, Asa Wallin, Jenny Jansson, Per Garberg, and Hans Postlind. "Introduction to in vitro Estimation of Metabolic Stability and Drug Interactions of New Chemical Entities in Drug Discovery and Development." Pharmacological Reports 58 (2006): 45372.

Bernadou, J., M. Bonnafous, G. Labat, P. Loiseau, and B. Meunier. "Biomimetic Oxidation of Acetaminophen and Ellipticine Derivatives with Water-soluble Metalloporphyrins Associated to Potassium Monopersulfate." Drug Metabolism and Disposition 19.2 (1990): 360-65.

Burgess, Steven J., Jane X. Kelly, Shawheen Shomloo, Sergio Wittlin, Reto Brun, Katherine Liebmann, David H. Peyton. "Synthesis, Structure-Activity Relationship, and Mode-of-Action Studies of Antimalarial Reversed Chloroquine Compounds." Journal of Medicinal Chemistry 53.17 (2010): 6477-489. Chinappi, Mauro, Allegra Via, Paolo Marcatili, and Anna Tramontano. "On the Mechanism of Chloroquine Resistance in Plasmodium Falciparum." Ed. Vladimir Brusic. PLoS ONE 5.11 (2010): E14064.

Danielson, P.B. "The Cytochrome P450 Superfamily: Biochemistry, Evolution and Drug Metabolism in Humans." Current Drug Metabolism 3.6 (2002): 561-97.

Gallup, John L., and Jeffrey D. Sachs. "The Economic Burden of Malaria." American Journal of Tropical Medicine and Hygiene. 64.1 (2001): 85-96.

Gedde, M.m., D.k. Davis, and W.h. Huestis. "Cytoplasmic pH and Human Erythrocyte Shape." Biophysical Journal 72.3 (1997): 1234-246. 
Johansson, Tove, Lars Weidolf, and Ulrik Jurva. "Mimicry of Phase I Drug Metabolism Novel Methods for Metabolite Characterization and Synthesis." Rapid Communications in Mass Spectrometry 21.14 (2007): 2323-331.

Jurva, Ulrik, Hakan V. Wikstrom, Lars Weidolf, and Andries P. Bruins. "Comparison Between Electrochemistry/mass Spectrometry and Cytochrome P450 Catalyzed Oxidation Reactions." Rapid Communications in Mass Spectrometry 17.8 (2003): 800-10.

Klein, E. "Antimalarial Drug Resistance: A Review of the Biology and Strategies to Delay Emergence and Spread." International Journal of Antimicrobial Agents 41.4 (2013): 311-17.

Kuhn, Yvonne, Petra Rohrbach, and Michael Lanzer. "Quantitative pH Measurements in Plasmodium Falciparum-infected Erythrocytes Using PHluorin." Cellular Microbiology 9.4 (2007): 1004-013.

World Health Organization. "Malaria." WHO. Web. 18 May 2014. http://www.who.int/topics/malaria/en/

Pishchany, Gleb, and Eric P. Skaar. "Taste for Blood: Hemoglobin as a Nutrient Source for Pathogens." Ed. Joseph Heitman. PLoS Pathogens 8.3 (2012): E1002535.

Roepe, Paul D. "The Molecular and Physiological Basis of Quinoline Drug Resistance in $P$. falciparum Malaria." Future Microbiology 4.4 (2009): 441-55.

Sherman, Irwin W. "The Life of Plasmodium: An Overview." Molecular Approaches to Malaria. Washington, DC: ASM, 2005. 3-11.

Wellems, Thomas, and Christopher Plowe. "Chloroquine-Resistant Malaria." The Journal of Infectious Diseases 184 (2001): 770-76.

Woggon, Wolf-D. "Structural and Functional Mimics of Cytochromes P450." The Ubiquitous Roles of Cytochrome P450 Proteins. Vol. 3. West Sussex: John Wiley and Sons, 2007. 27-55. 


\section{Appendix A: NMR spectra of PL69}

NMR spectra of PL69 in deuterated methanol

400 NMR

${ }^{1} \mathrm{H}$ NMR \# of scans $=16$

${ }^{13} \mathrm{C}$ NMR \# of scans $=256$

\begin{tabular}{|c|c|c|c|c|}
\hline Assignment & ${ }^{1} \mathrm{H}$ shift (ppm) & Multiplicity & Integration & ${ }^{13} \mathrm{C}$ shift (ppm) \\
\hline a & - & - & - & 162.17 \\
\hline$b$ & - & - & - & 152.71 \\
\hline $\mathrm{C}$ & 8.34 & $\mathrm{~d}$ & 1 & 152.47 \\
\hline d & 8.5 & $\mathrm{dq}$ & 2 & 149.98 \\
\hline $\mathrm{e}$ & - & - & - & 149.63 \\
\hline$f$ & 7.76 & ddd & 2 & 138.62 \\
\hline g & - & - & - & 136.31 \\
\hline $\mathrm{h}$ & 7.77 & d & 1 & 127.63 \\
\hline $\mathrm{i}$ & 7.44 & ddd & 1 & 126.09 \\
\hline j & 8.03 & $d$ & 1 & 124.29 \\
\hline $\mathrm{k}$ & 7.53 & d & 2 & 124.06 \\
\hline 1 & 7.26 & ddd & 2 & 123.94 \\
\hline $\mathrm{m}$ & - & - & - & 118.75 \\
\hline $\mathrm{n}$ & 6.49 & d & 1 & 99.59 \\
\hline 0 & 5.24 & $\mathrm{~s}$ & 1 & 66.59 \\
\hline$p$ & 2.44 & $\mathrm{t}$ & 2 & 57.63 \\
\hline$q$ & 2.4 & $\mathrm{~m}$ & 1 & 53.87 \\
\hline$r$ & 2.95 & d & 2 & 53.5 \\
\hline$r^{\prime}$ & 1.92 & $\mathrm{~m}$ & 2 & 53.5 \\
\hline $\mathrm{S}$ & 3.36 & $\mathrm{t}$ & 2 & 42.89 \\
\hline $\mathrm{t}$ & 1.95 & $\mathrm{~m}$ & 2 & 33.01 \\
\hline $\mathrm{t}^{\prime}$ & 1.55 & $\mathrm{~m}$ & 2 & 33.01 \\
\hline $\mathrm{u}$ & 1.91 & $\mathrm{~m}$ & 2 & 26.13 \\
\hline
\end{tabular}<smiles>C[C@@H]1CC(NC(c2ccccn2)c2ccccn2)CCN1CCCNc1ccnc2cc(Cl)ccc12</smiles> 

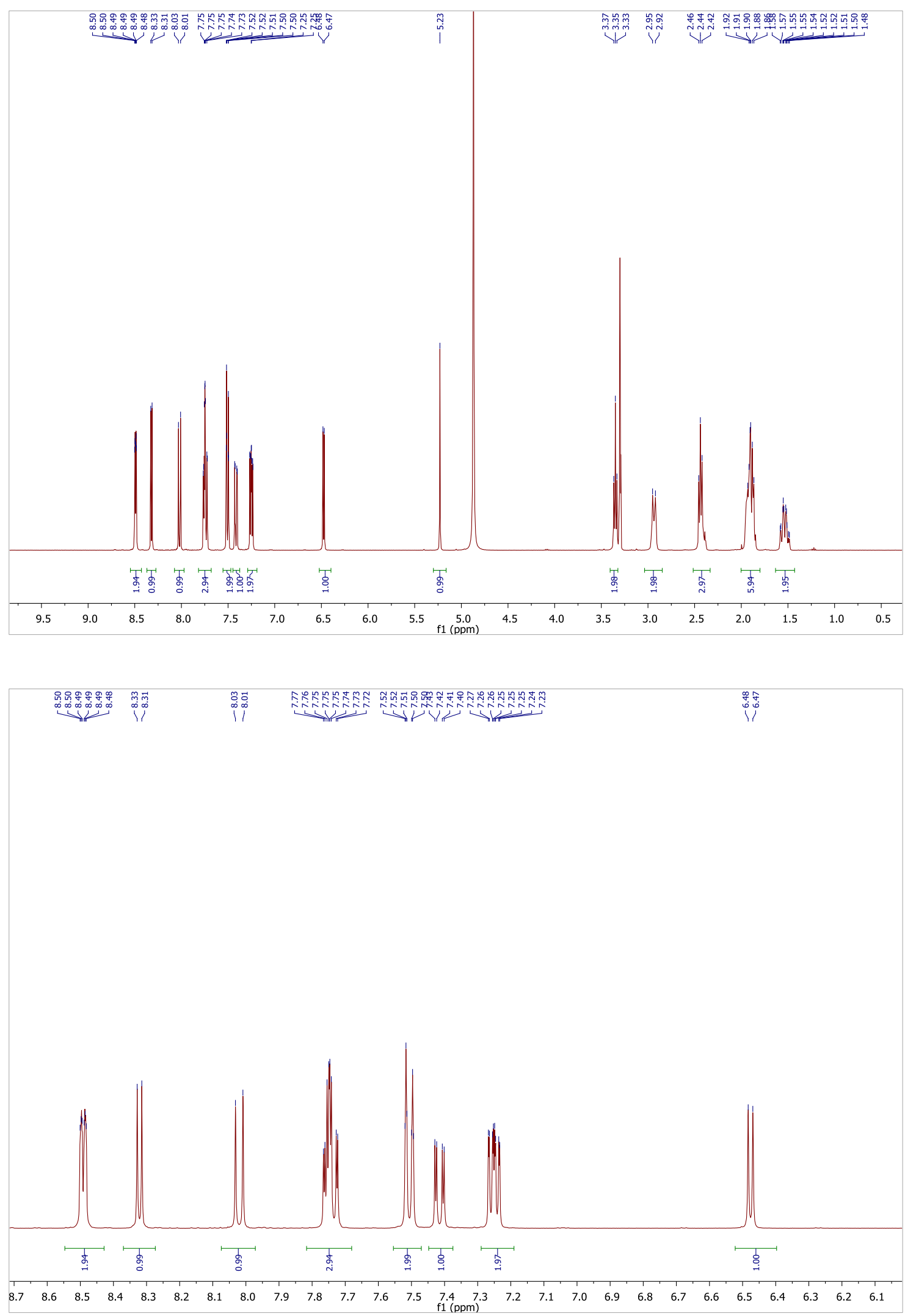

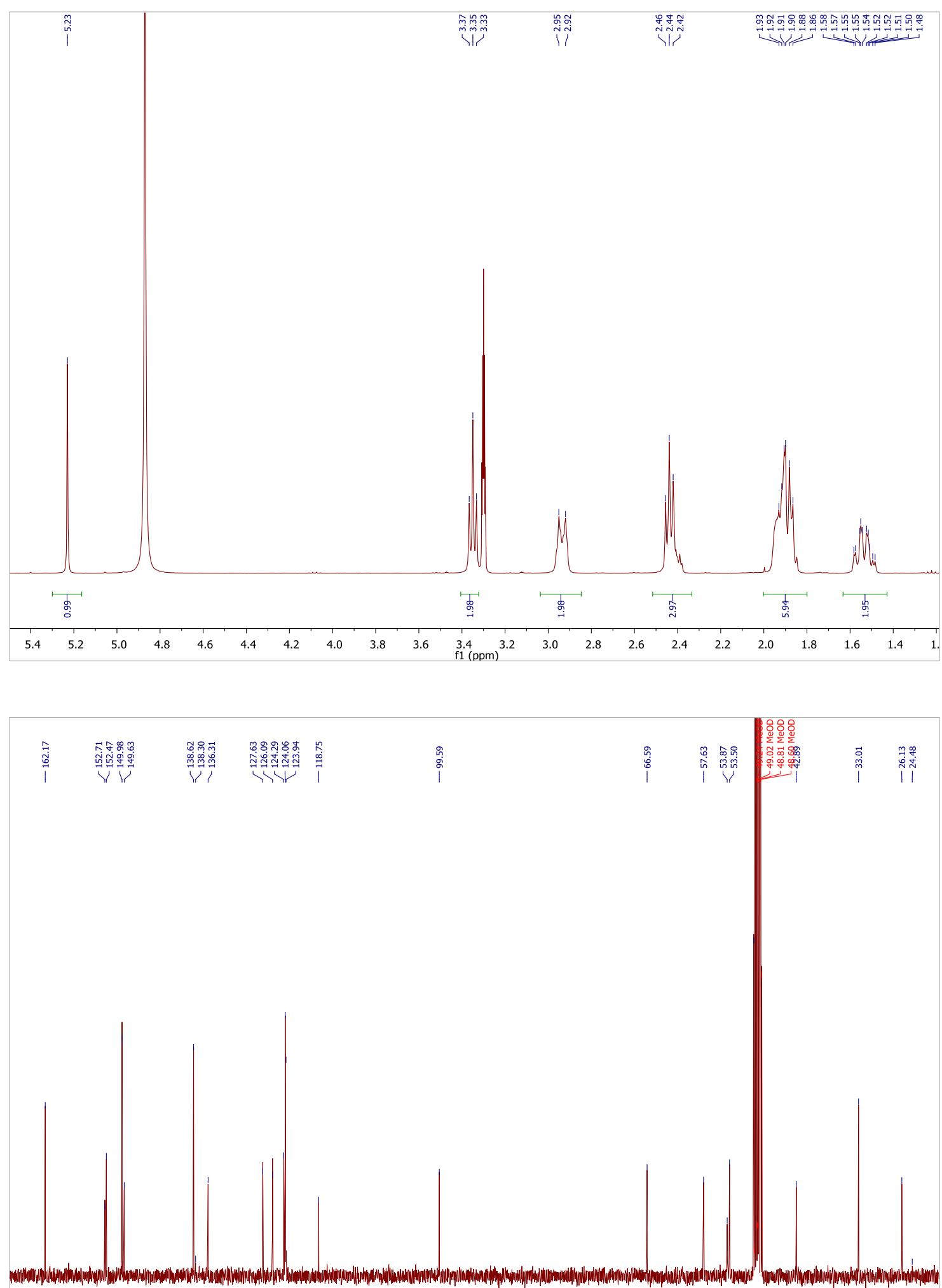

$\begin{array}{lllllllllllllllllllllllllllll}165 & 160 & 155 & 150 & 145 & 140 & 135 & 130 & 125 & 120 & 115 & 110 & 105 & 100 & 95 & 90 & 85 & 80 & 75 & 70 & 65 & 60 & 55 & 50 & 45 & 40 & 35 & 30 & 25\end{array}$ 


\section{Appendix B: NMR spectra of PL16}

NMR spectra of PL16 in deuterated methanol

$400 \mathrm{NMR}$

${ }^{1} \mathrm{H}$ NMR \# of scans $=16$

${ }^{13} \mathrm{C}$ NMR \# of scans=256

\begin{tabular}{|c|c|c|c|c|}
\hline Assignment & ${ }^{1} \mathrm{H}$ shift $(\mathrm{ppm})$ & Multiplicity & Integration & 13C shift (ppm) \\
\hline a & - & - & - & 152.86 \\
\hline b & 8.34 & $\mathrm{~d}$ & 1 & 152.22 \\
\hline c & - & - & - & 149.41 \\
\hline d & - & - & - & 136.39 \\
\hline e & 7.77 & d & 1 & 127.41 \\
\hline f & 7.39 & dd & 1 & 126.03 \\
\hline g & 8.05 & d & 1 & 124.24 \\
\hline h & - & - & - & 118.71 \\
\hline i & 6.54 & d & 1 & 99.58 \\
\hline j & 3.74 & t & 2 & 60.77 \\
\hline k & 3.47 & t & 2 & 41.26 \\
\hline I & 1.96 & p & 2 & 32.12 \\
\hline
\end{tabular}

Assignment of PL16 NMR chemical shifts<smiles>OC(O)CCNc1ccnc2cc(Cl)ccc12</smiles> 


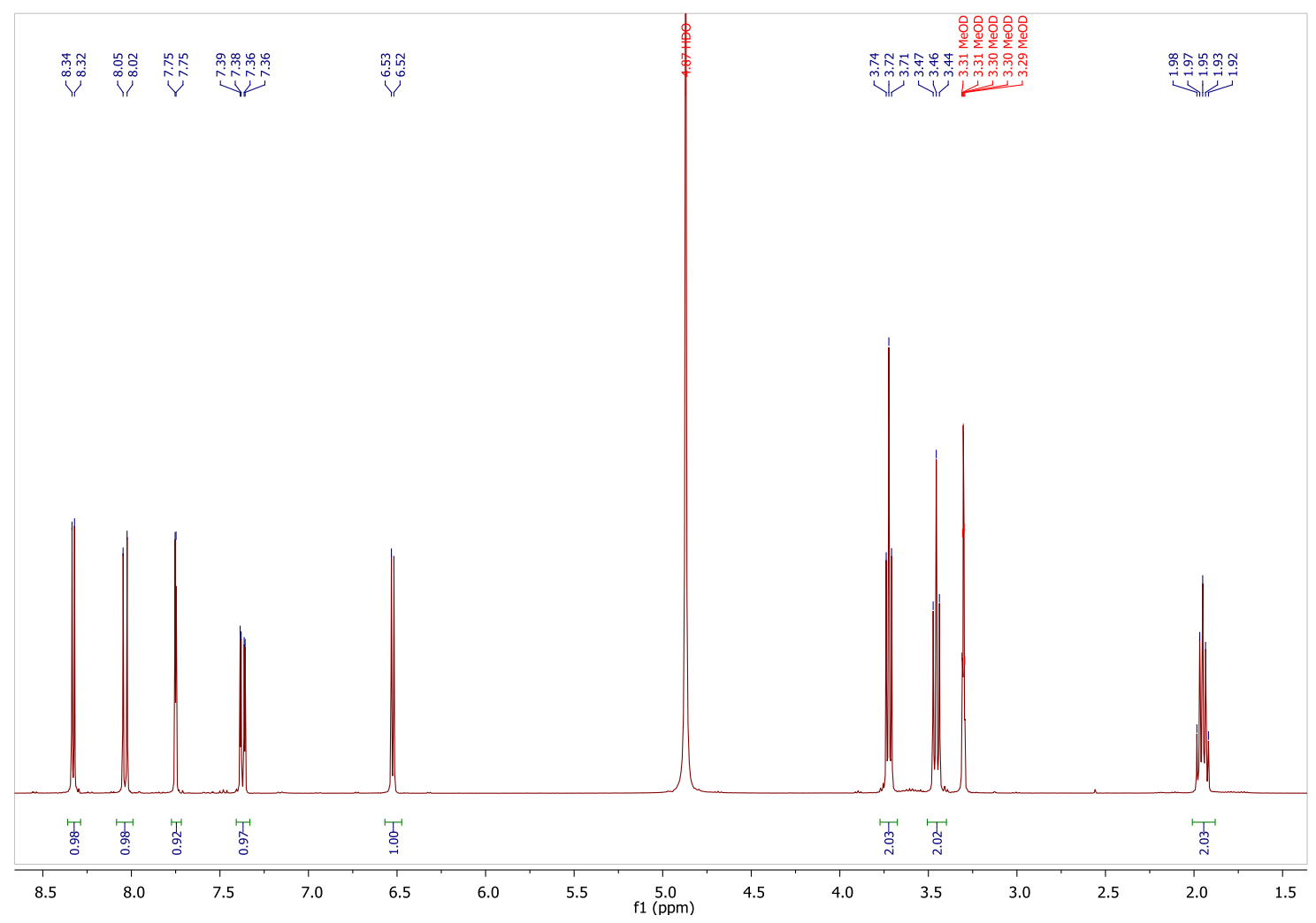



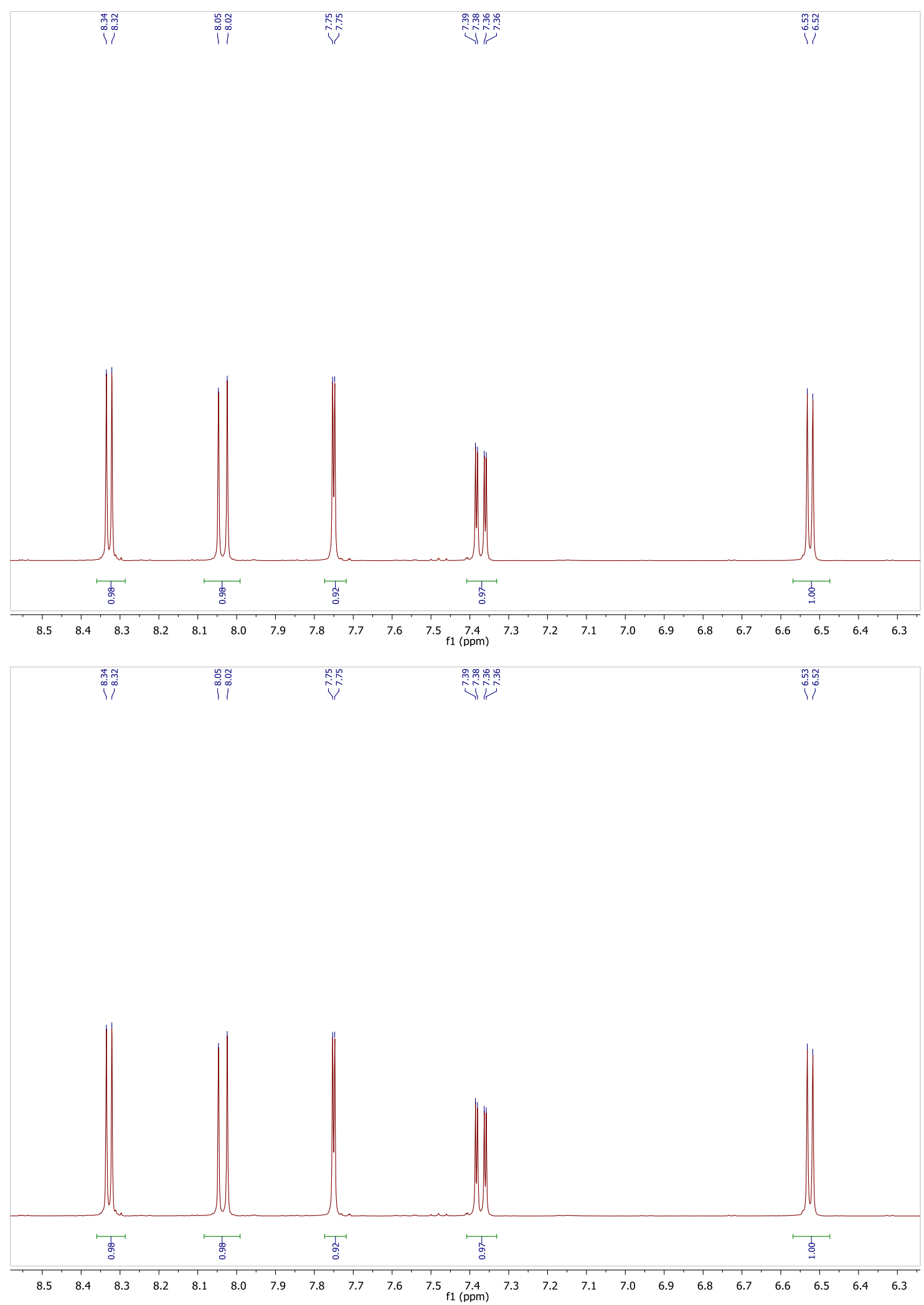


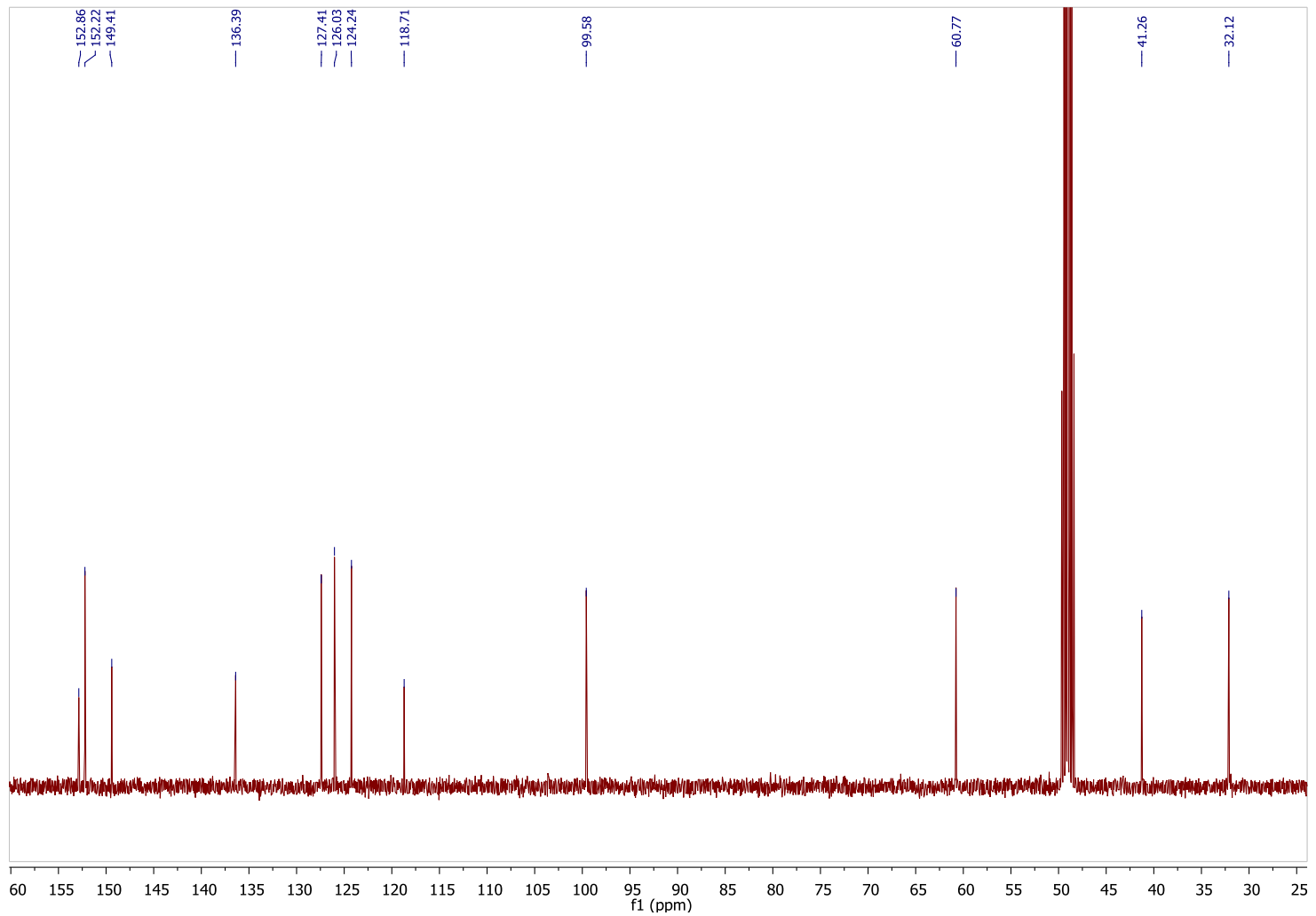




\section{Appendix C: NMR spectra of oxidation products}

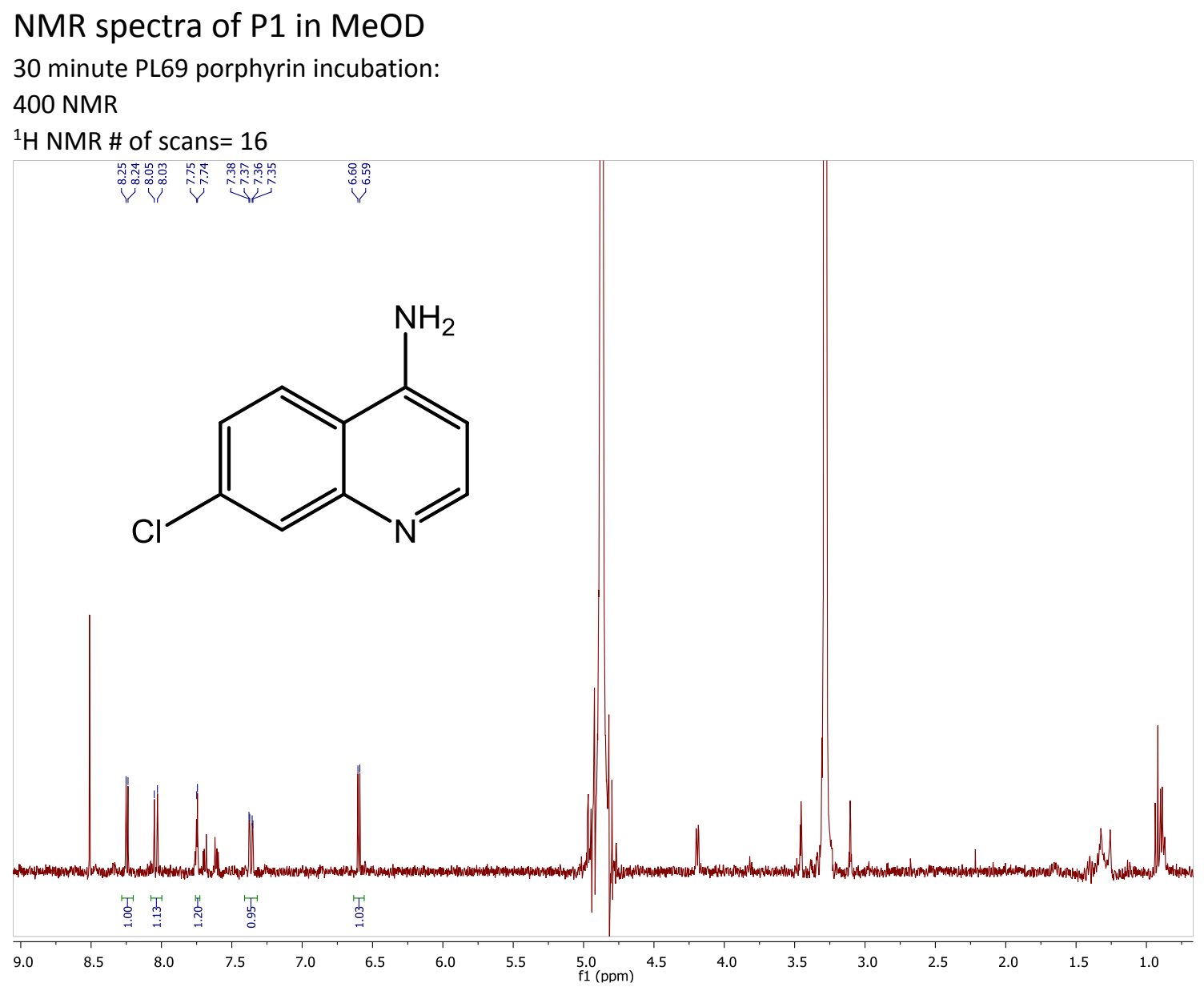




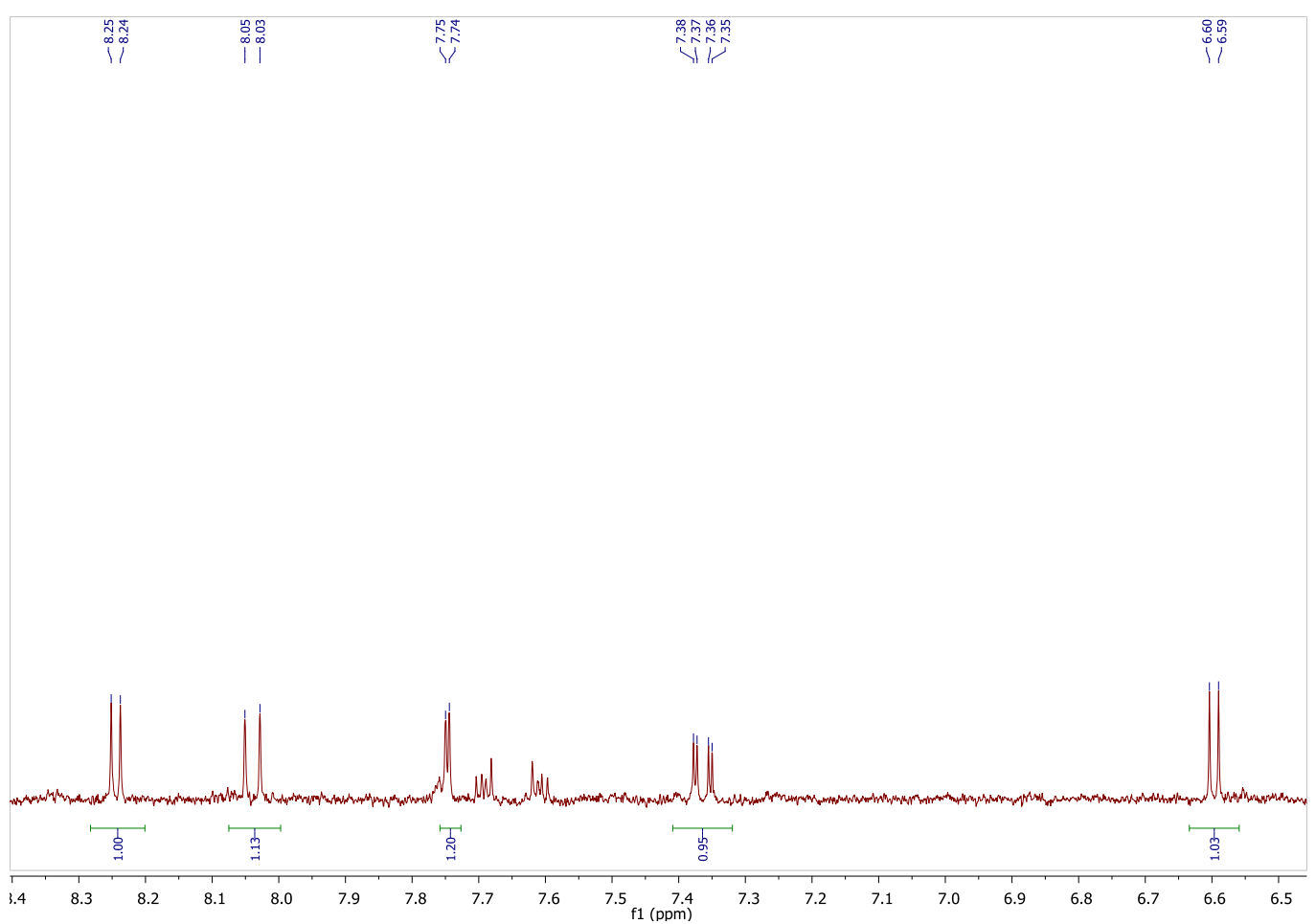

30 minute PL16 incubation:

400 NMR

${ }^{1} \mathrm{H}$ NMR \# of scans $=256$

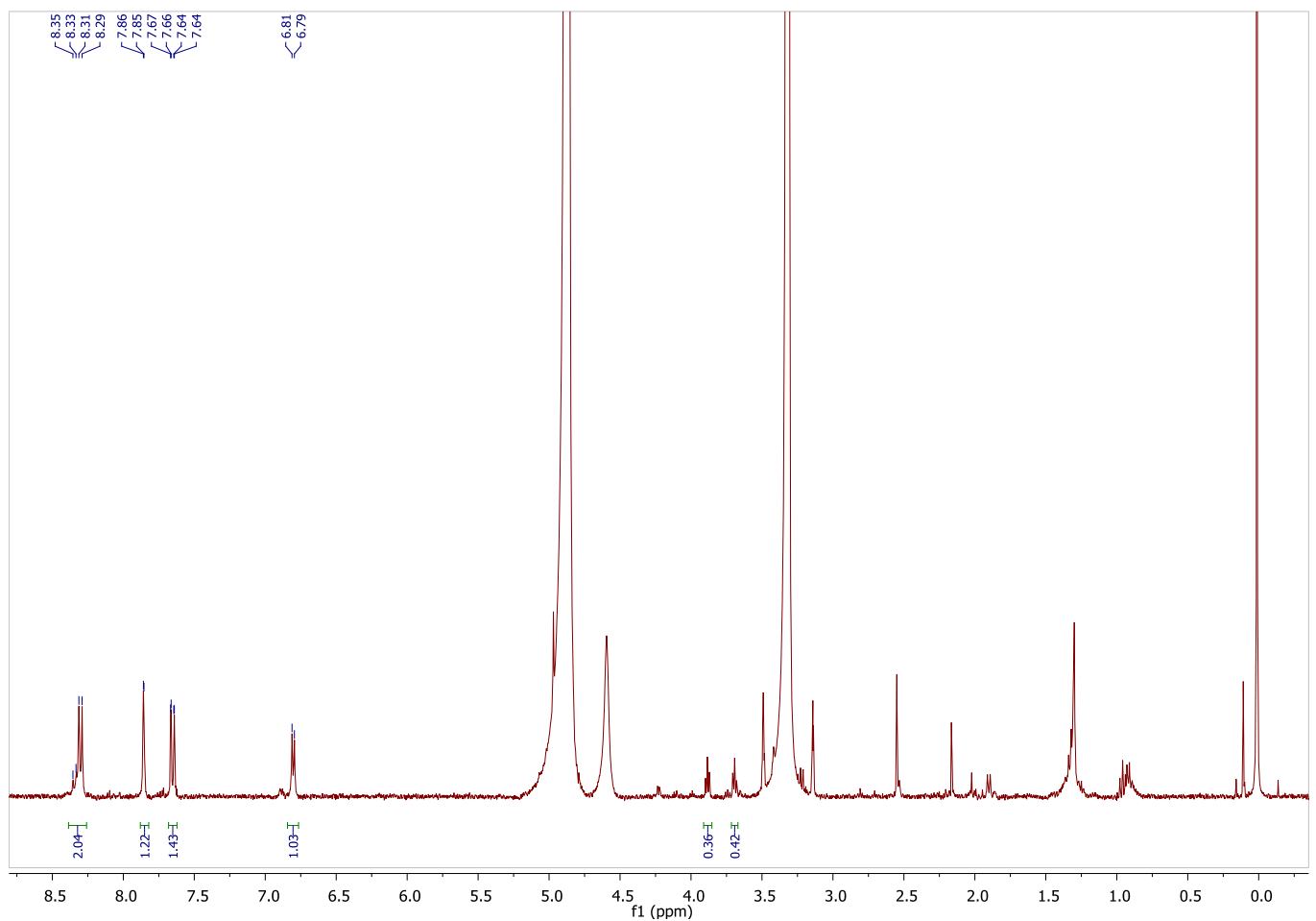




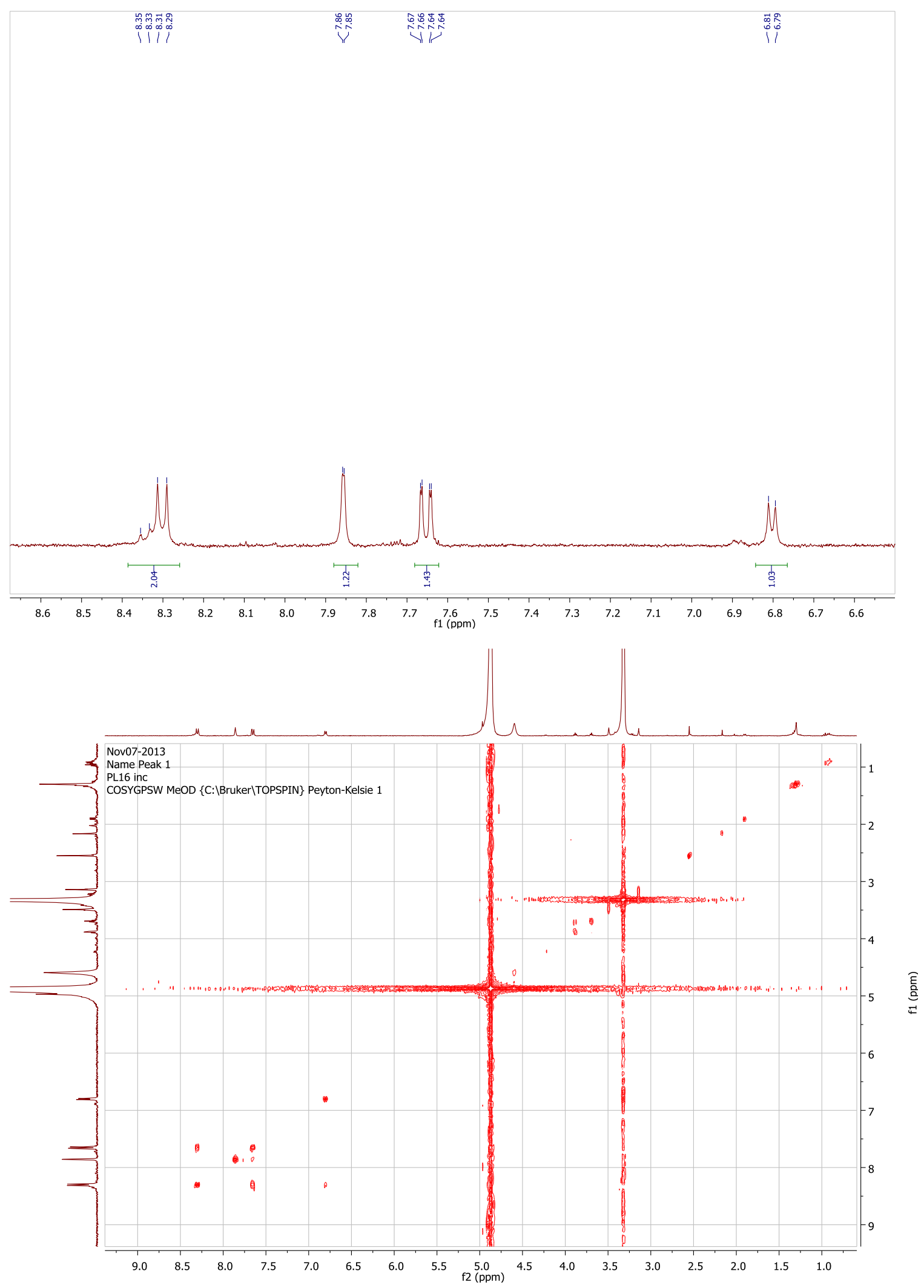




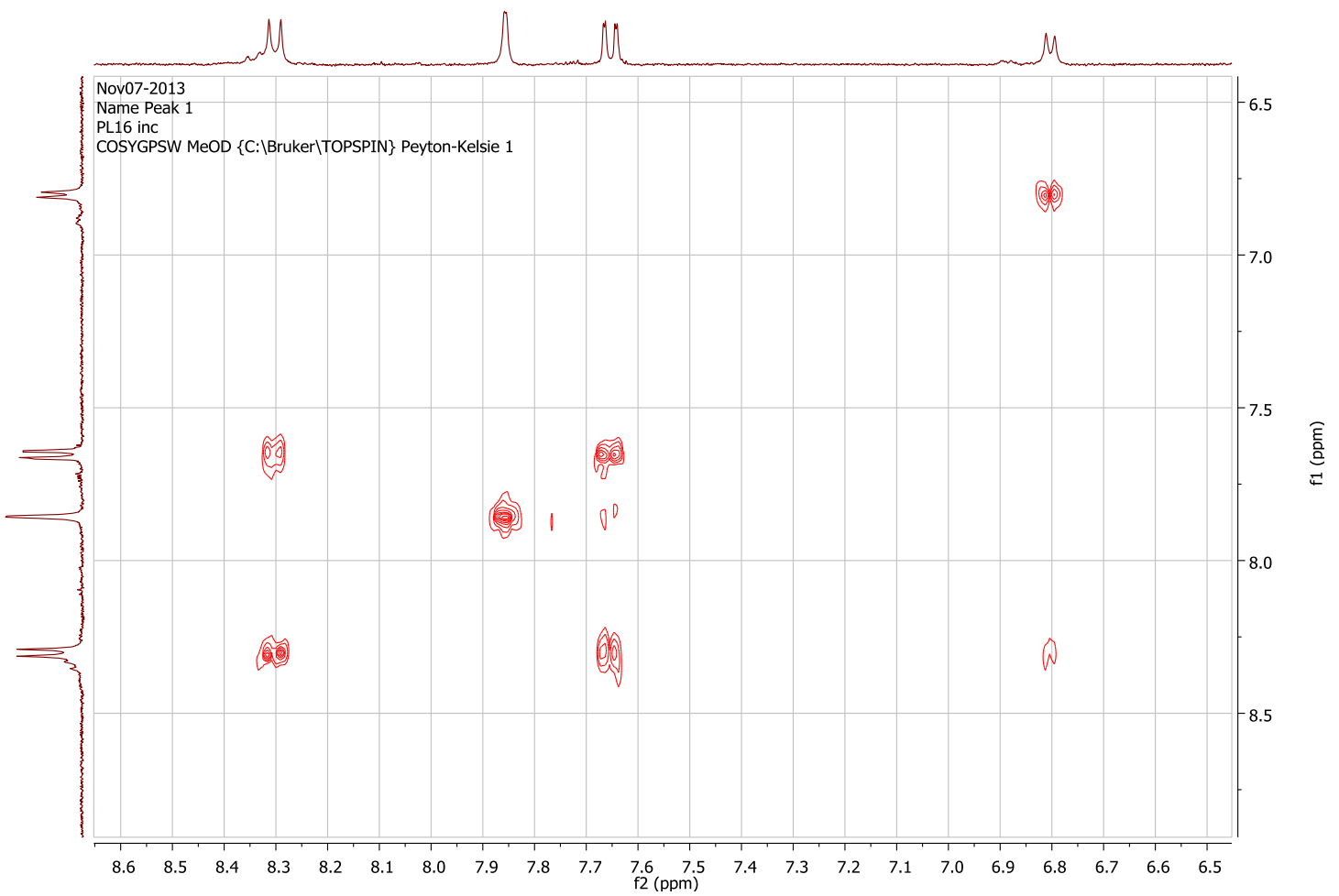


NMR spectra of P2A and P2B

$400 \mathrm{NMR}$

${ }^{1} \mathrm{H}$ NMR \# of scans $=512$

P2A in MeOD:

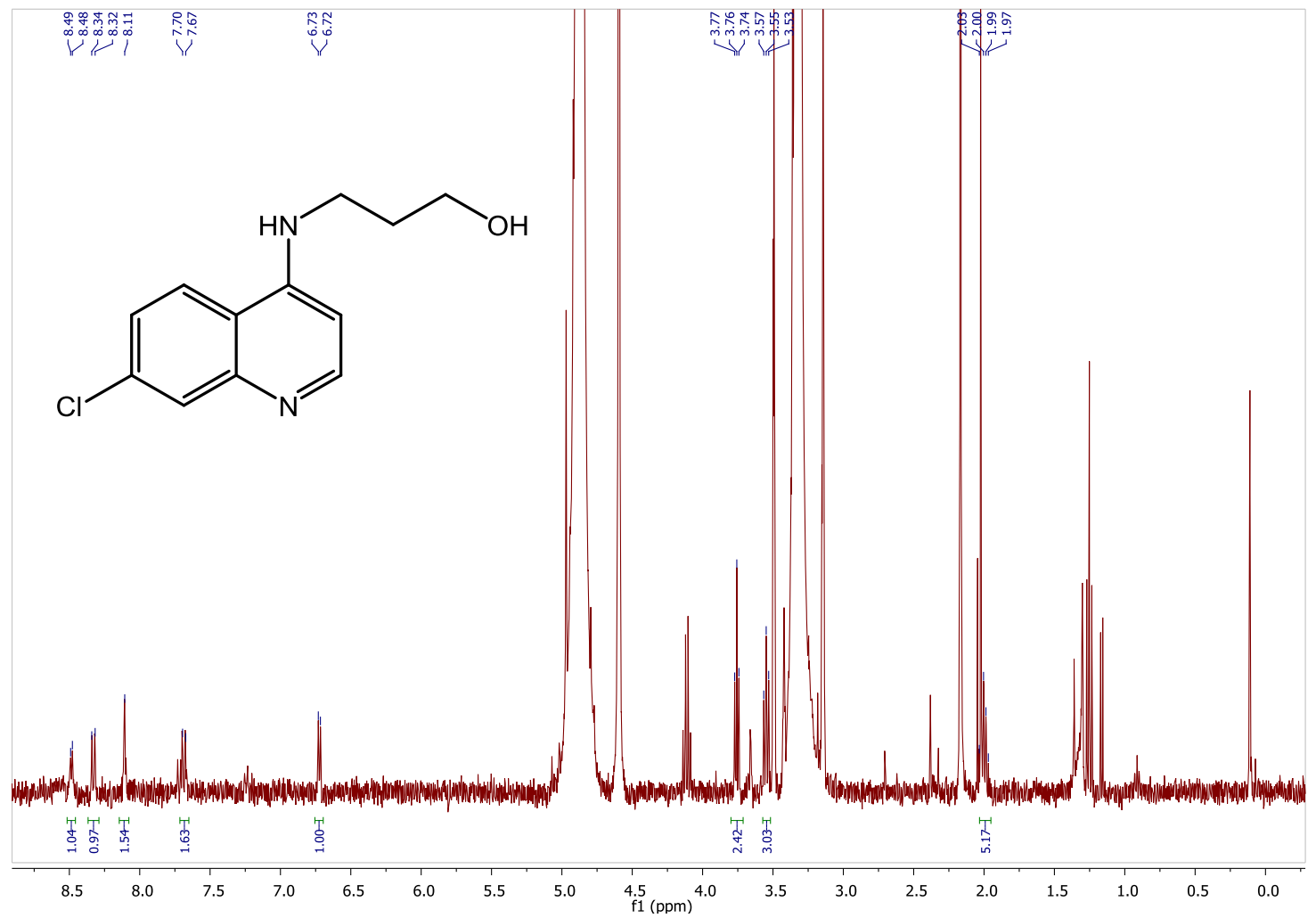



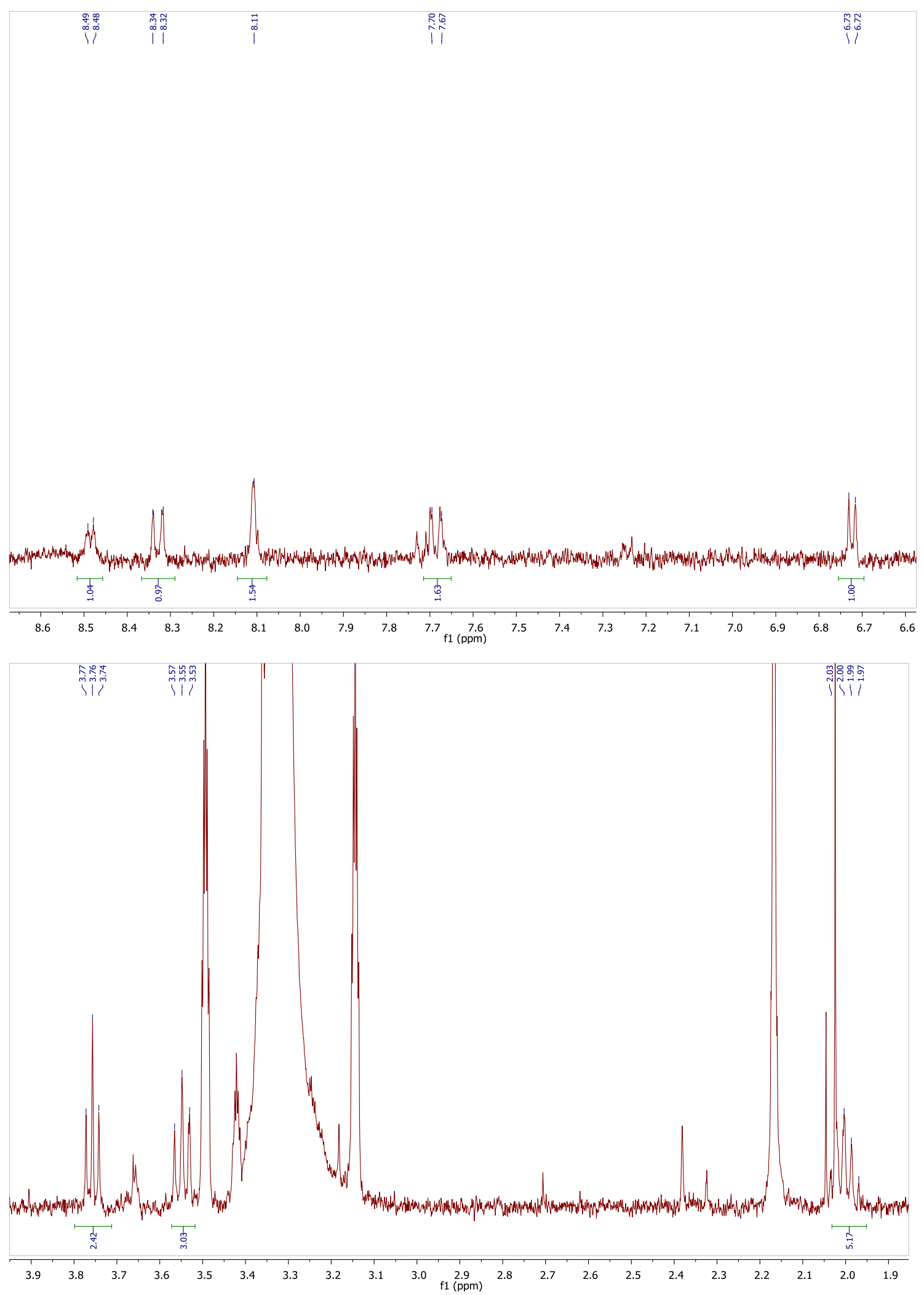
$\mathrm{P} 2 \mathrm{~B}$ in MeOD:
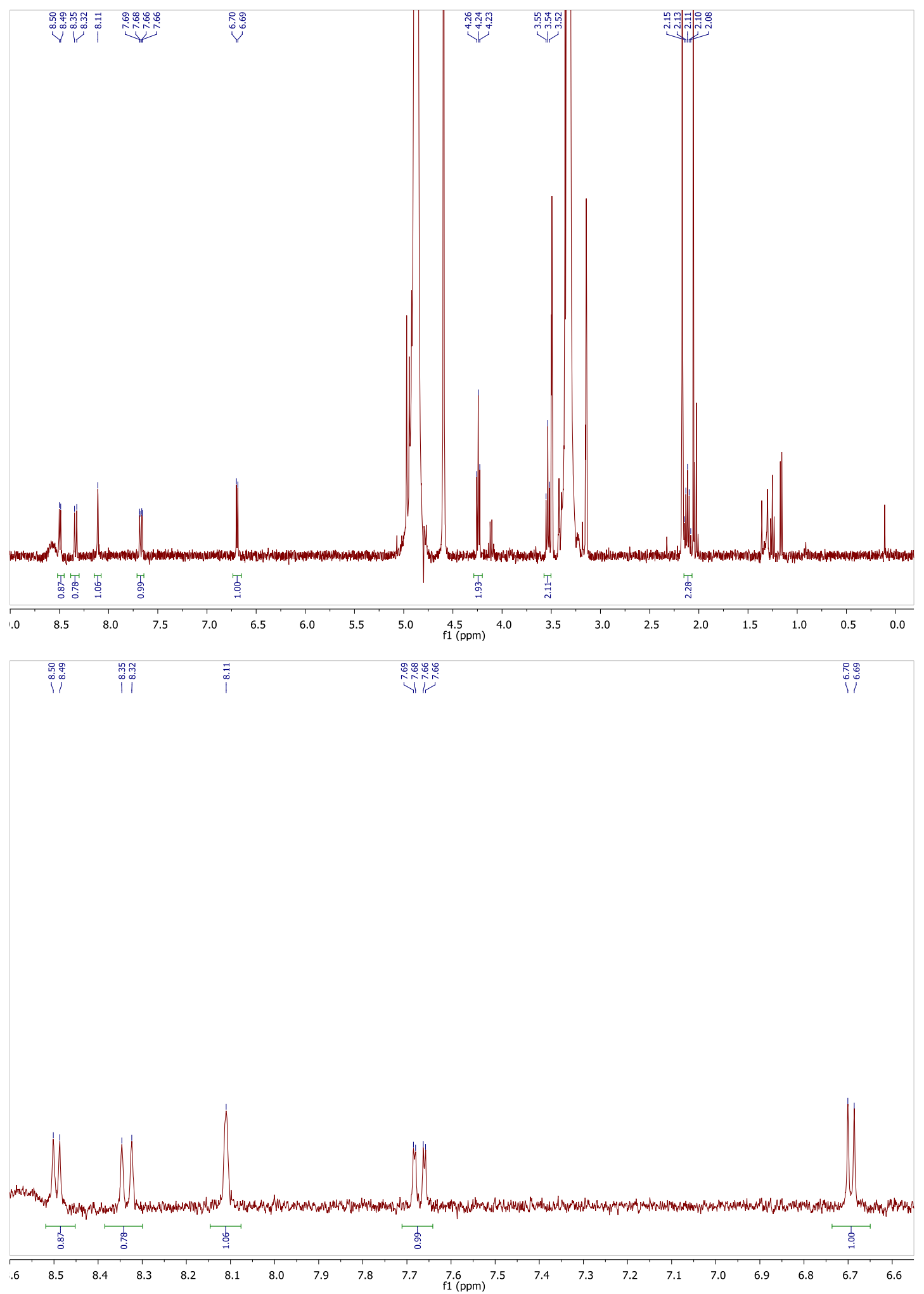


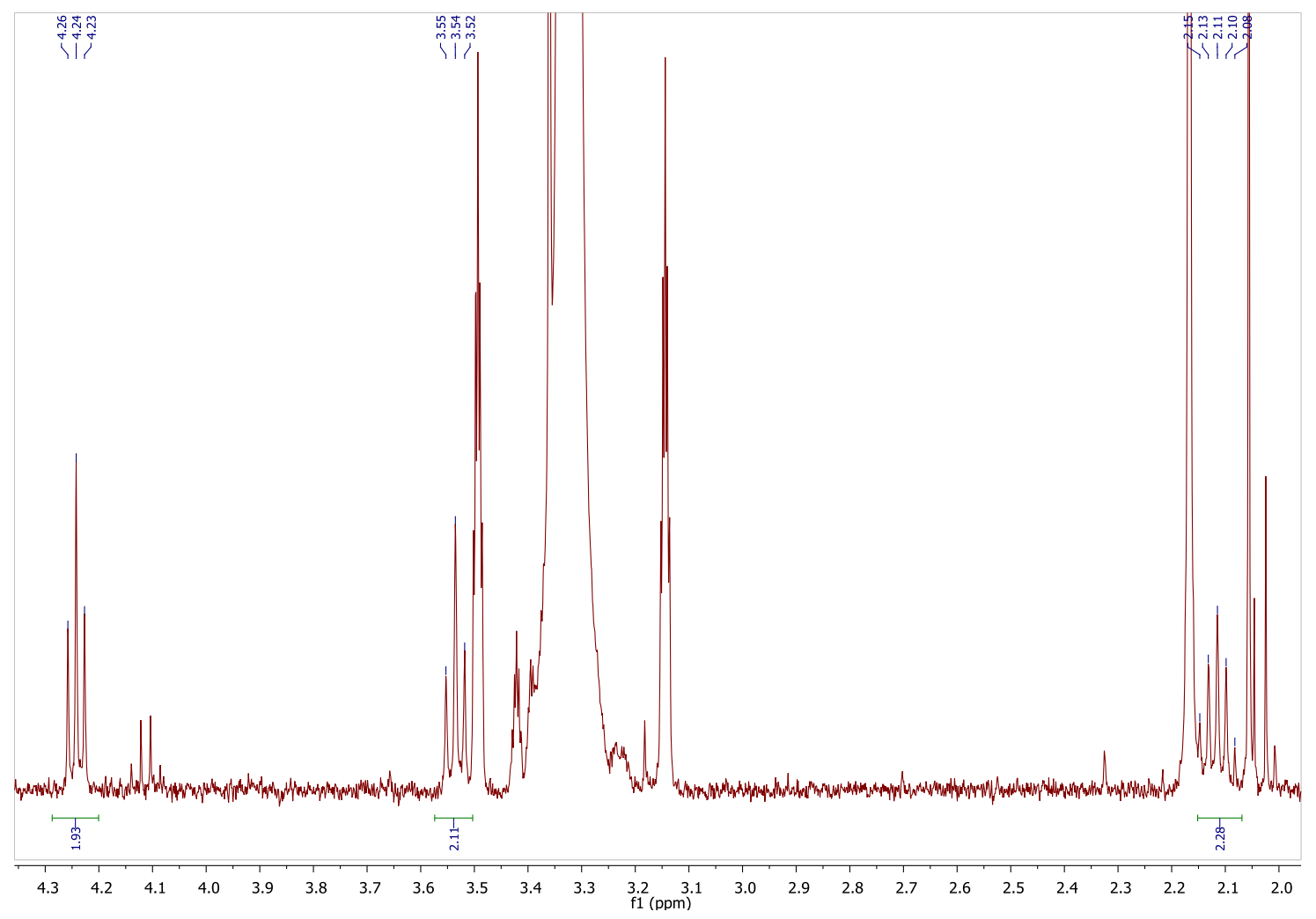

69 


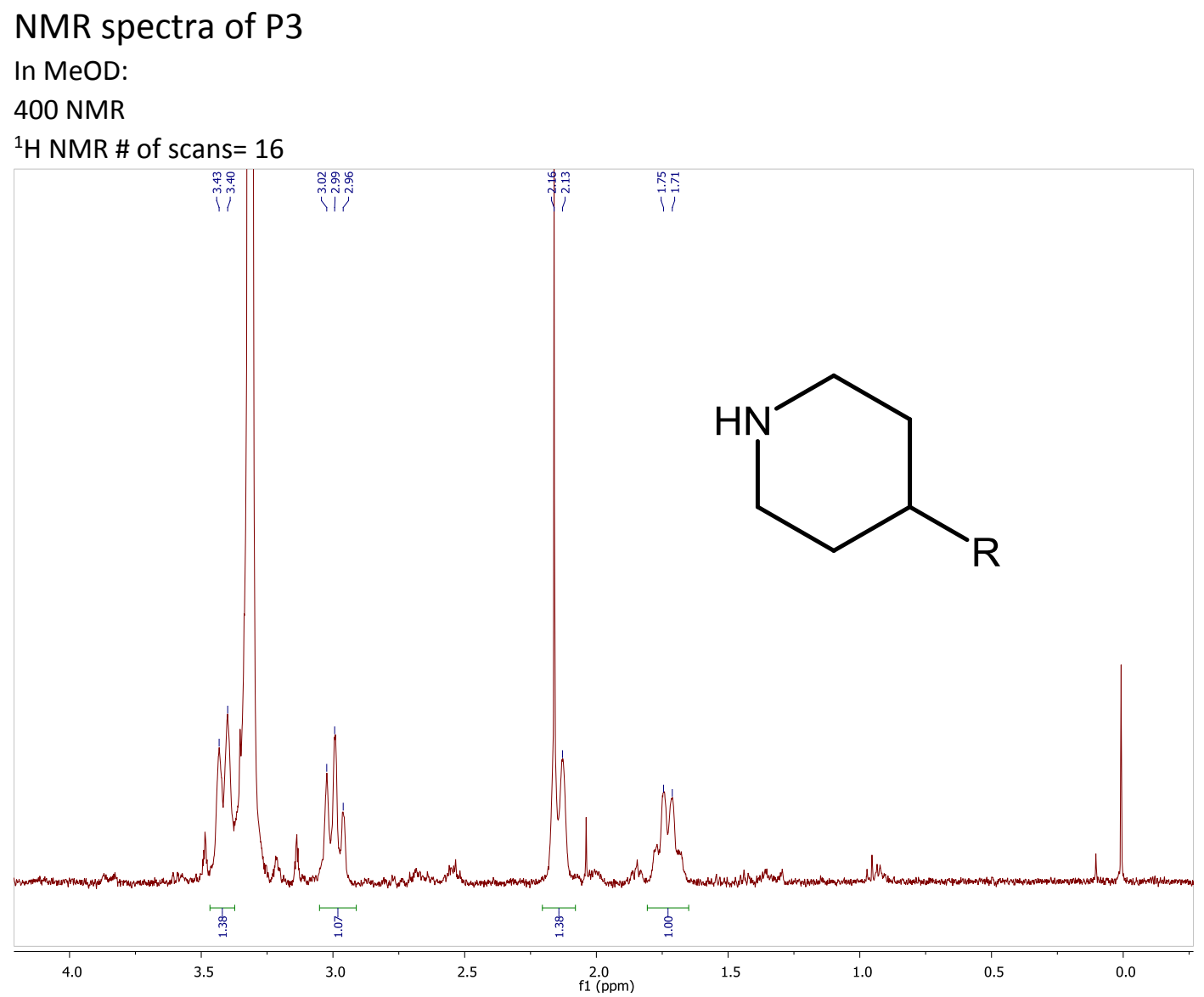


In $\mathrm{D}_{2} \mathrm{O}$ :

400 NMR

${ }^{1} \mathrm{H}$ NMR \# of scans $=256$
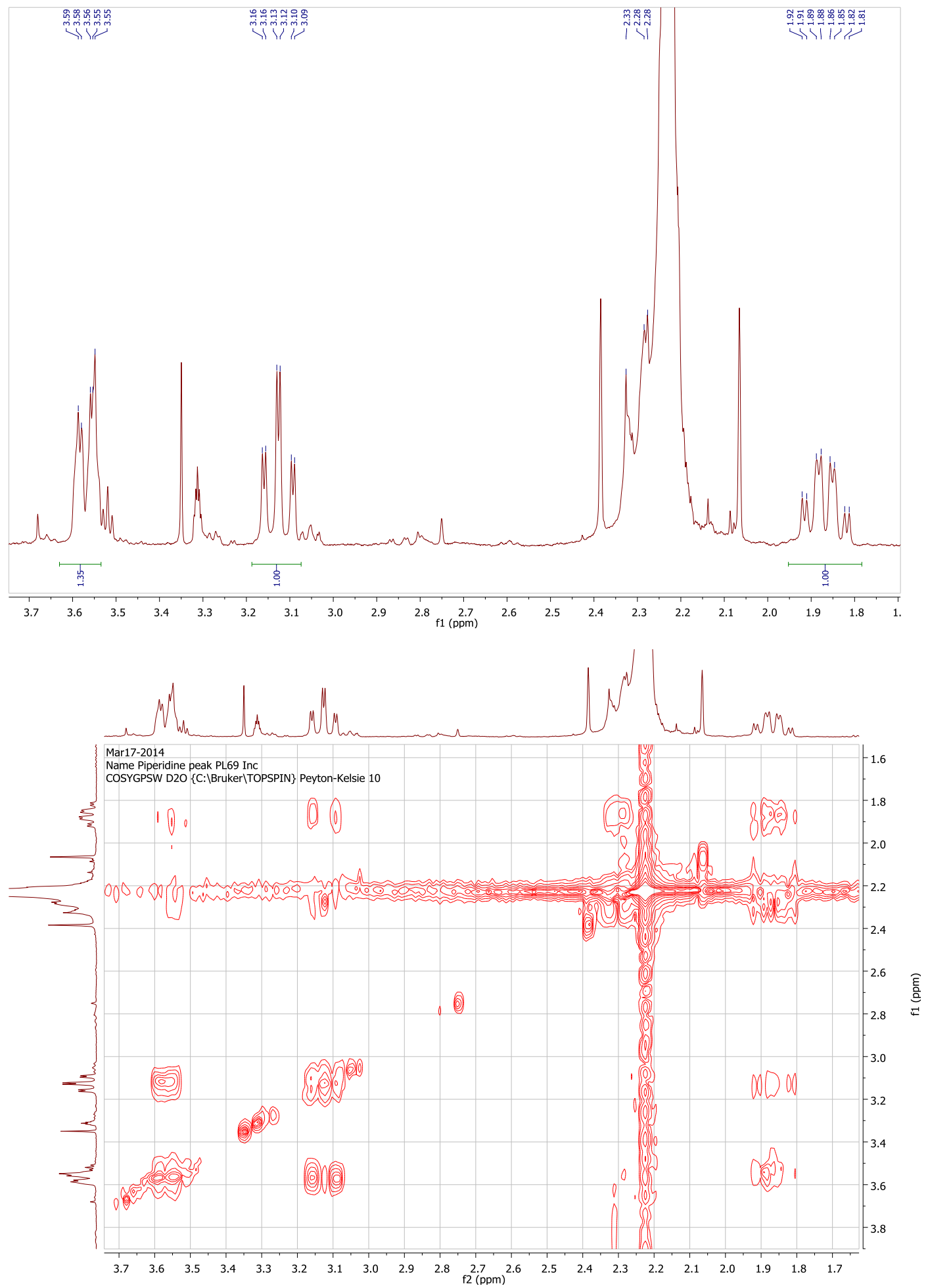


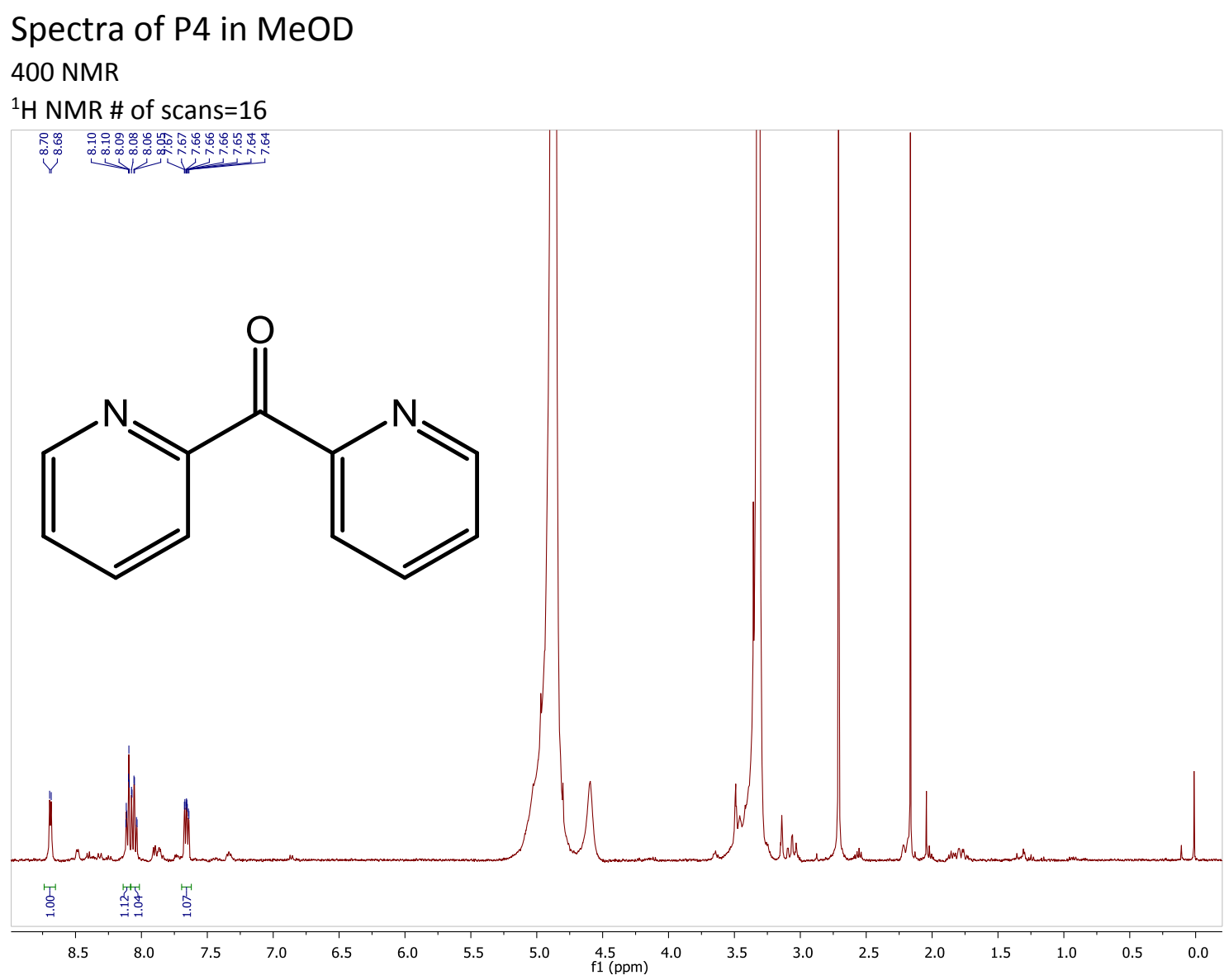




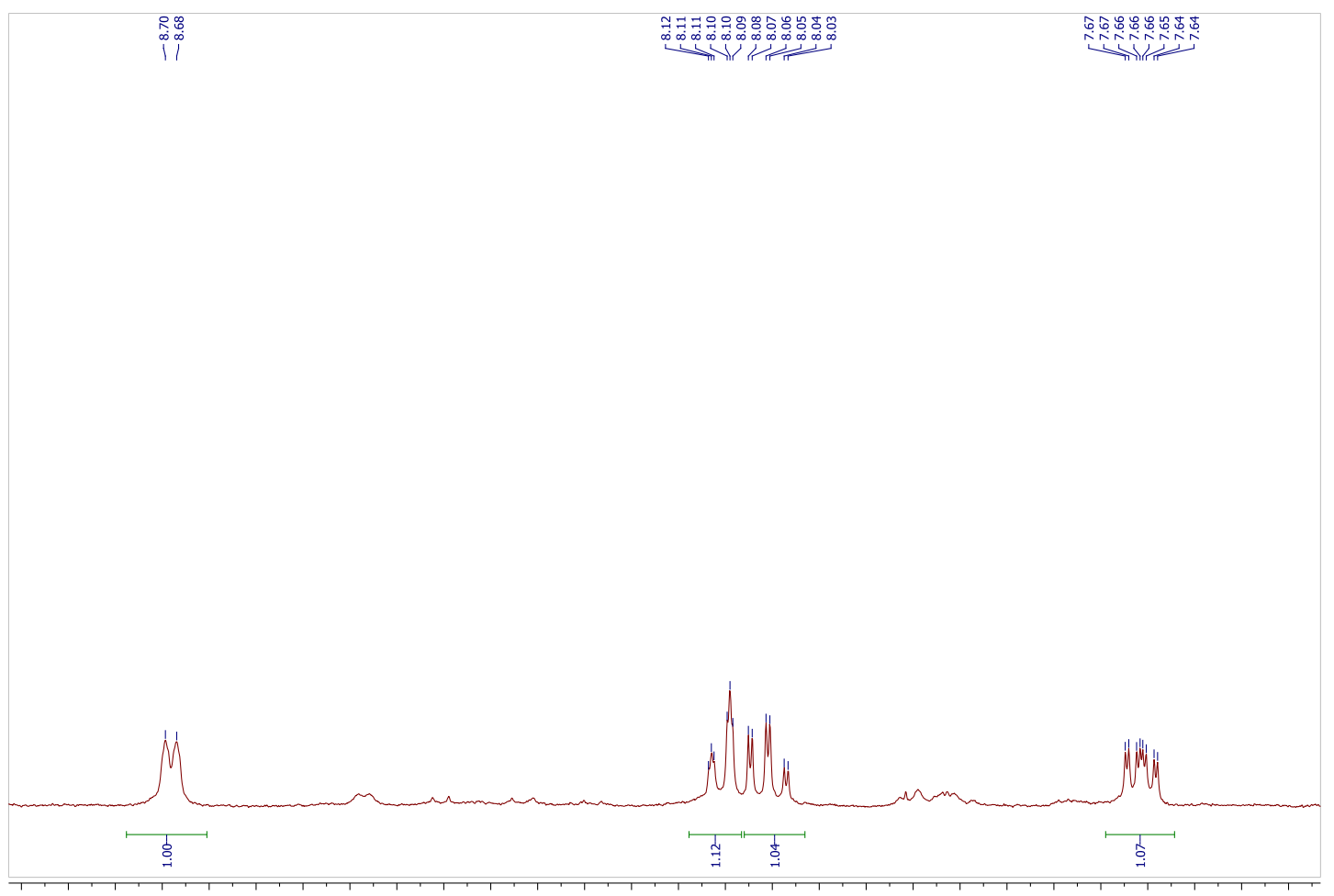

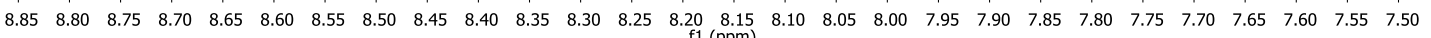
f1 (ppm)

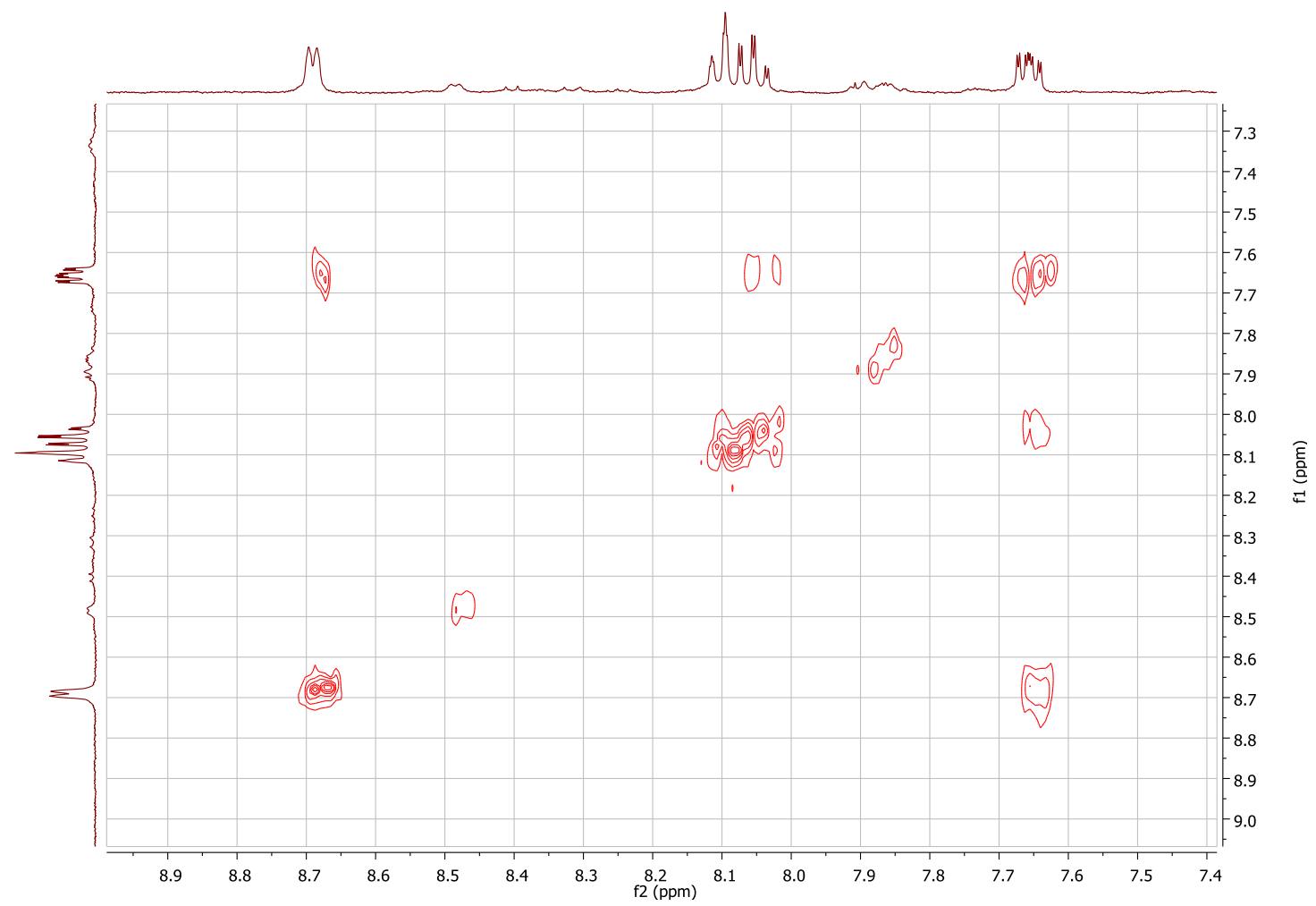




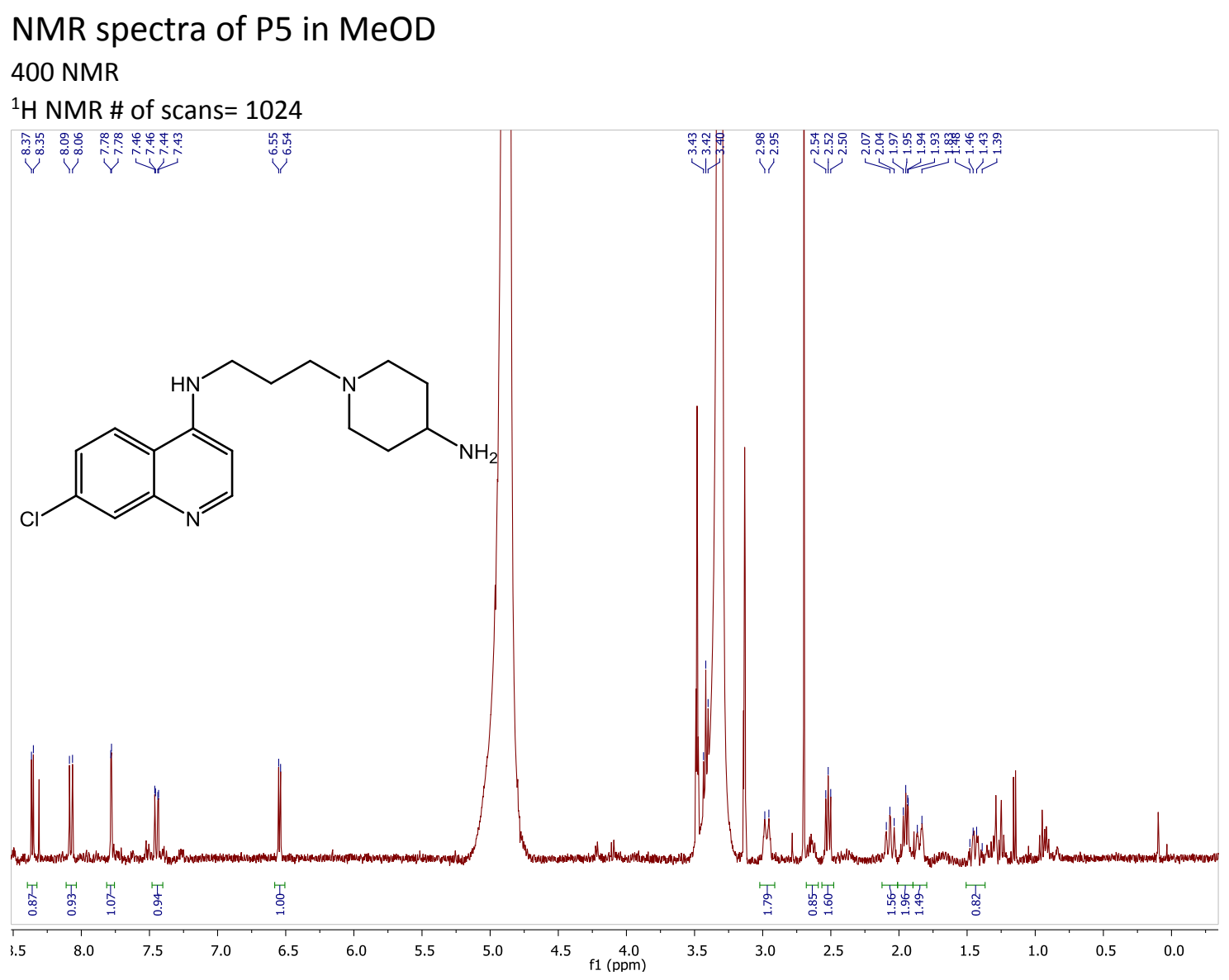



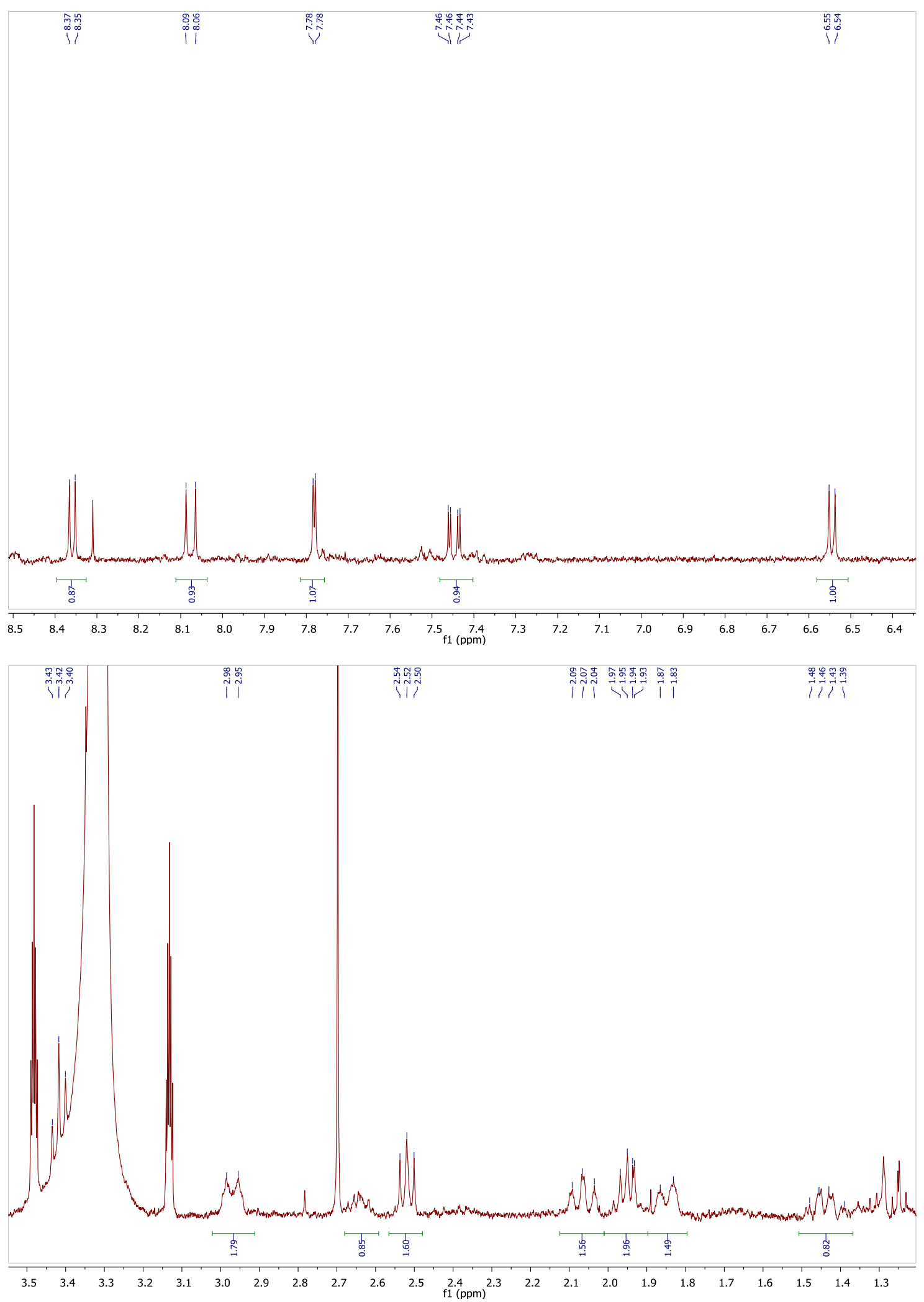


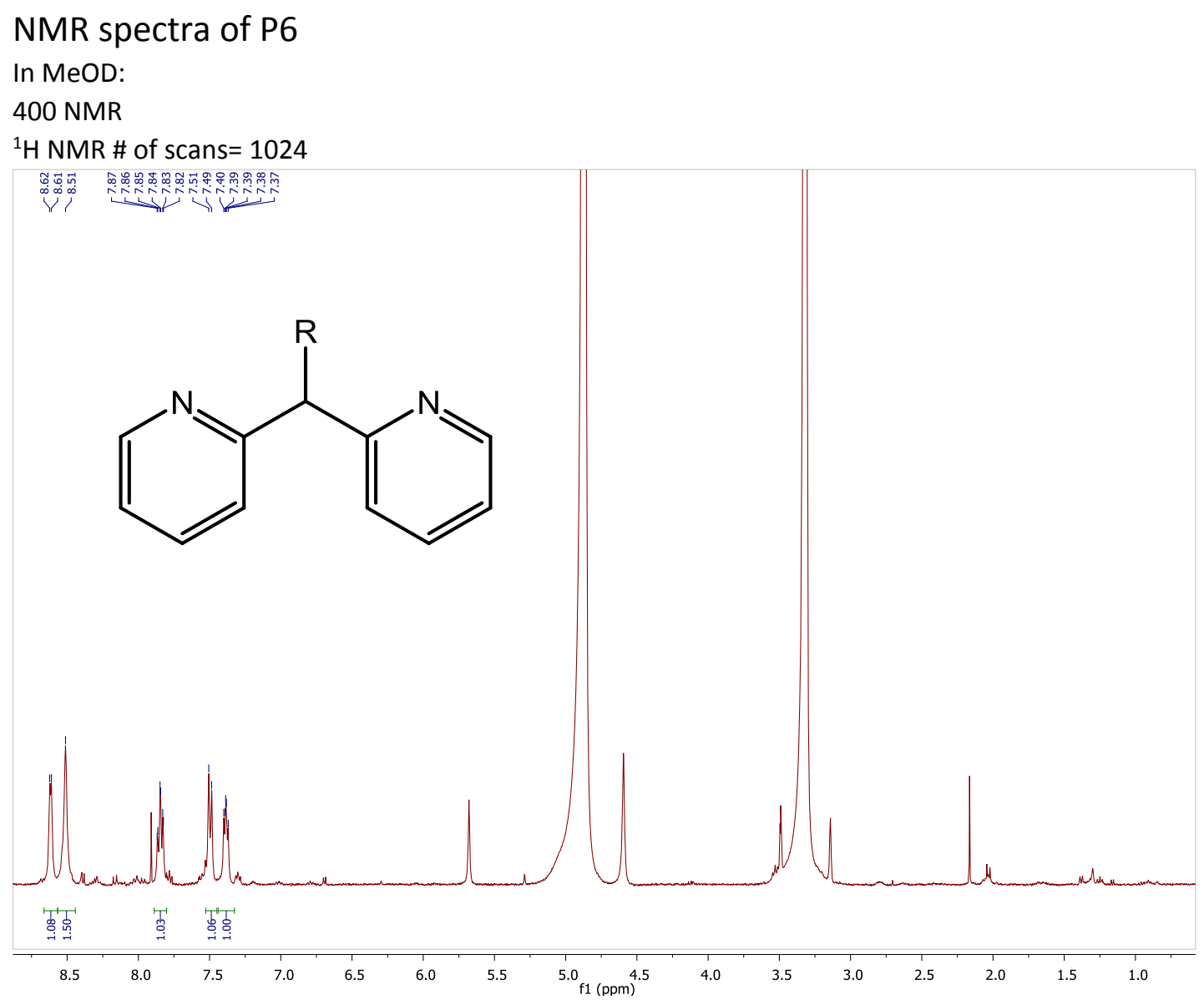



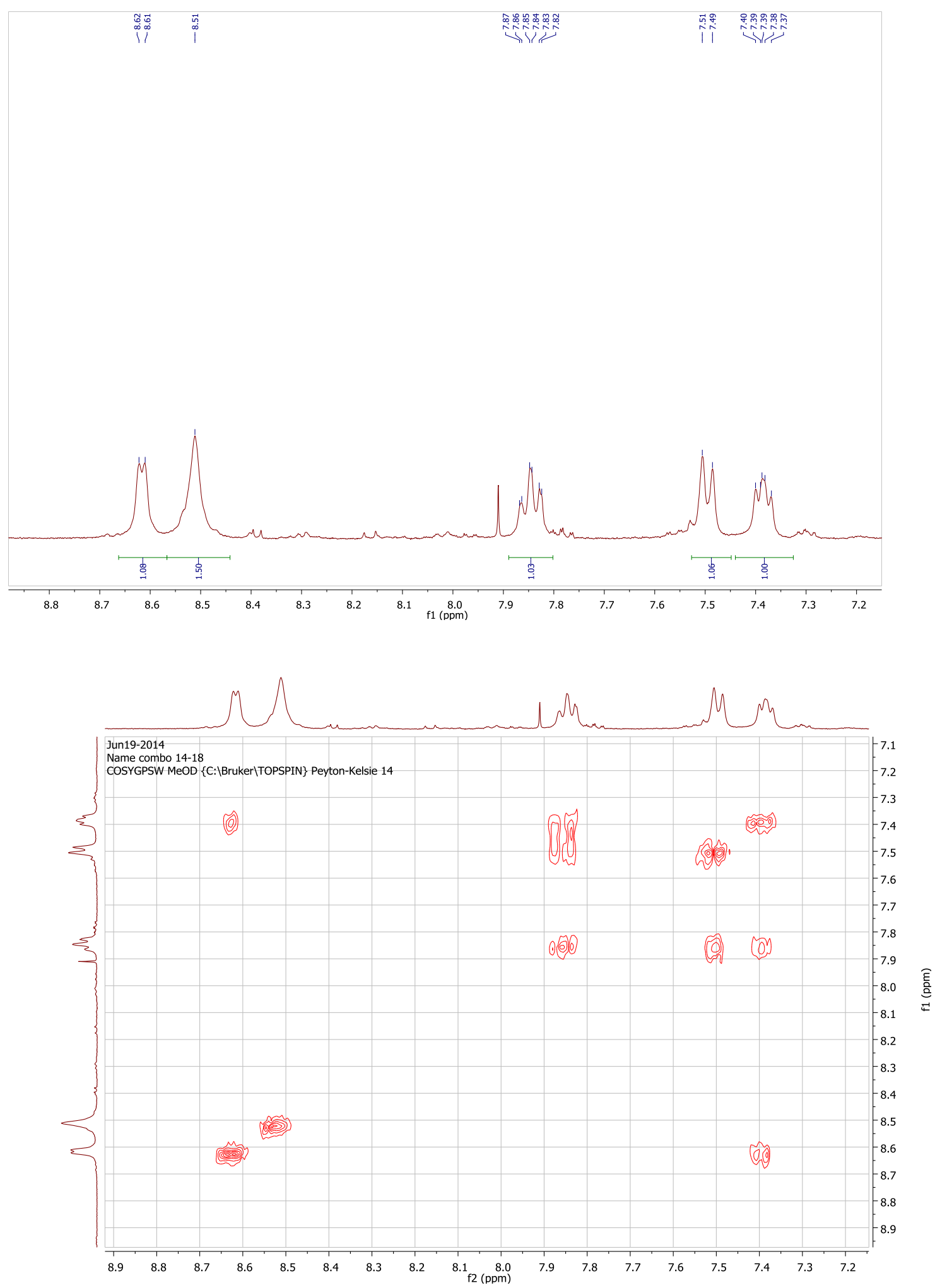
In DMSO-d6:

400 NMR

${ }^{1} \mathrm{H}$ NMR \# of scans $=1024$

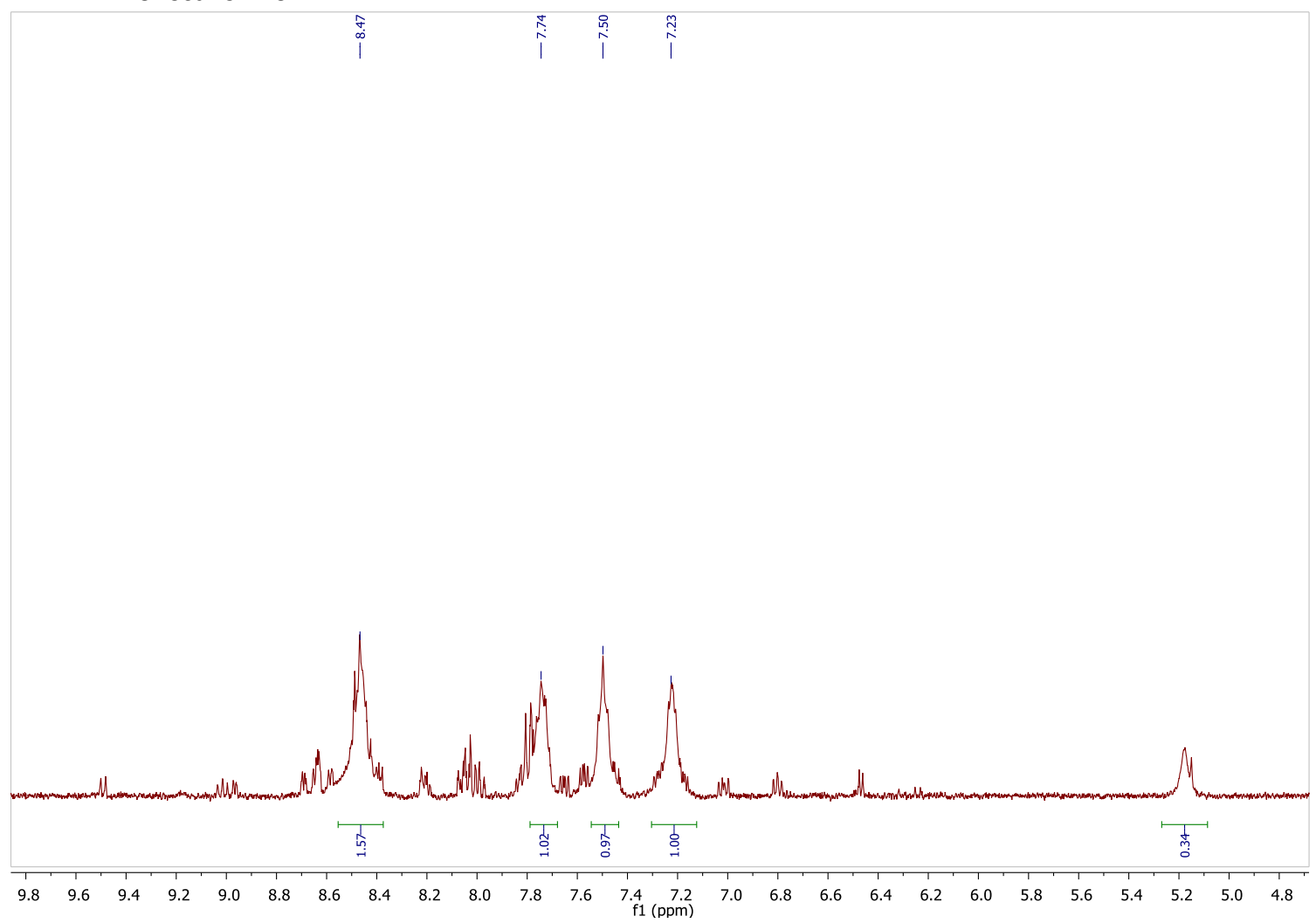


In DMSO-d6 with NaOD:

400 NMR

${ }^{1} \mathrm{H}$ NMR \# of scans $=1024$
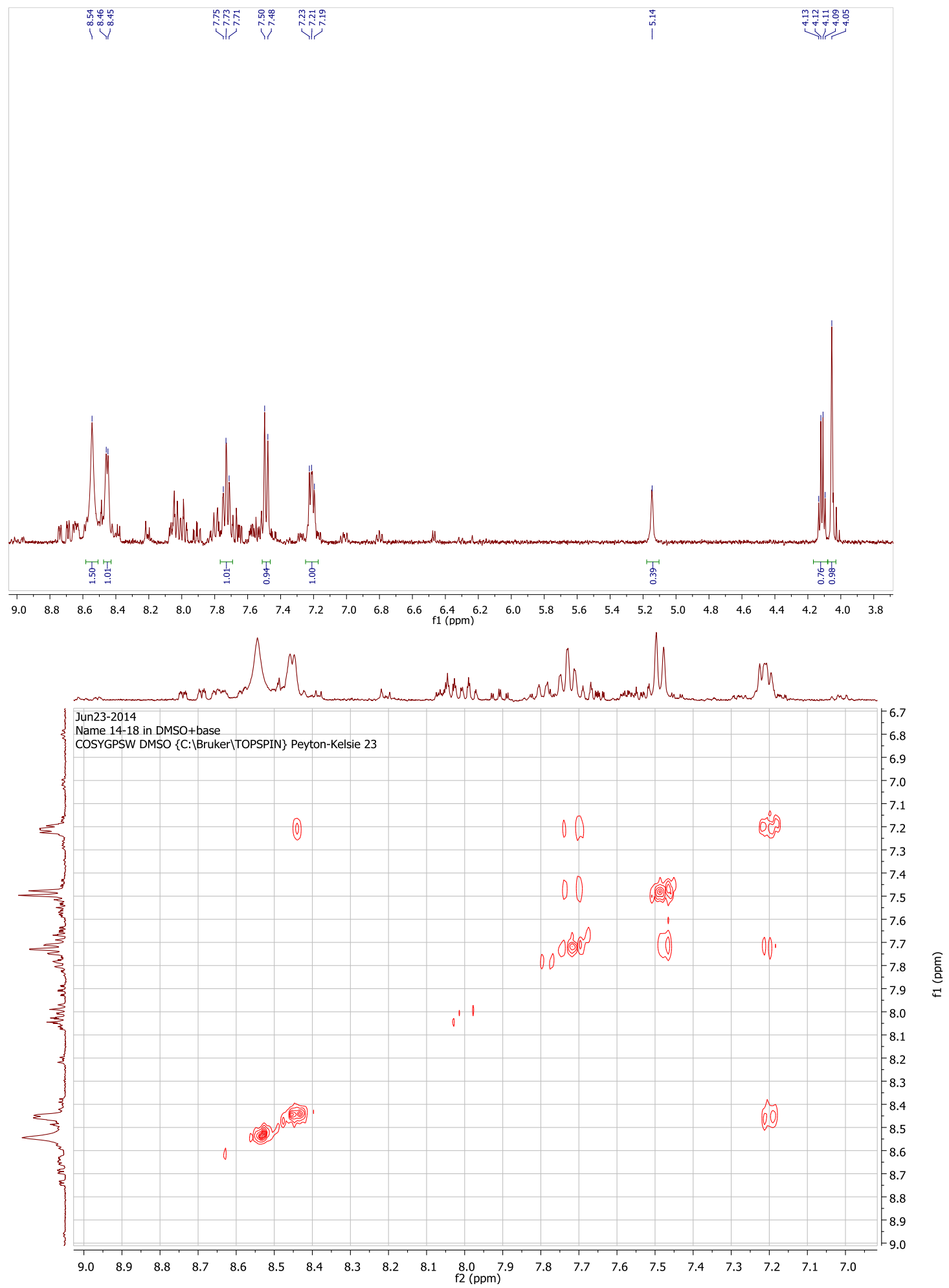
NMR spectra of P7 in $\mathrm{CDCl}_{3}$

$400 \mathrm{NMR}$

${ }^{1} \mathrm{H}$ NMR \# of scans $=256$

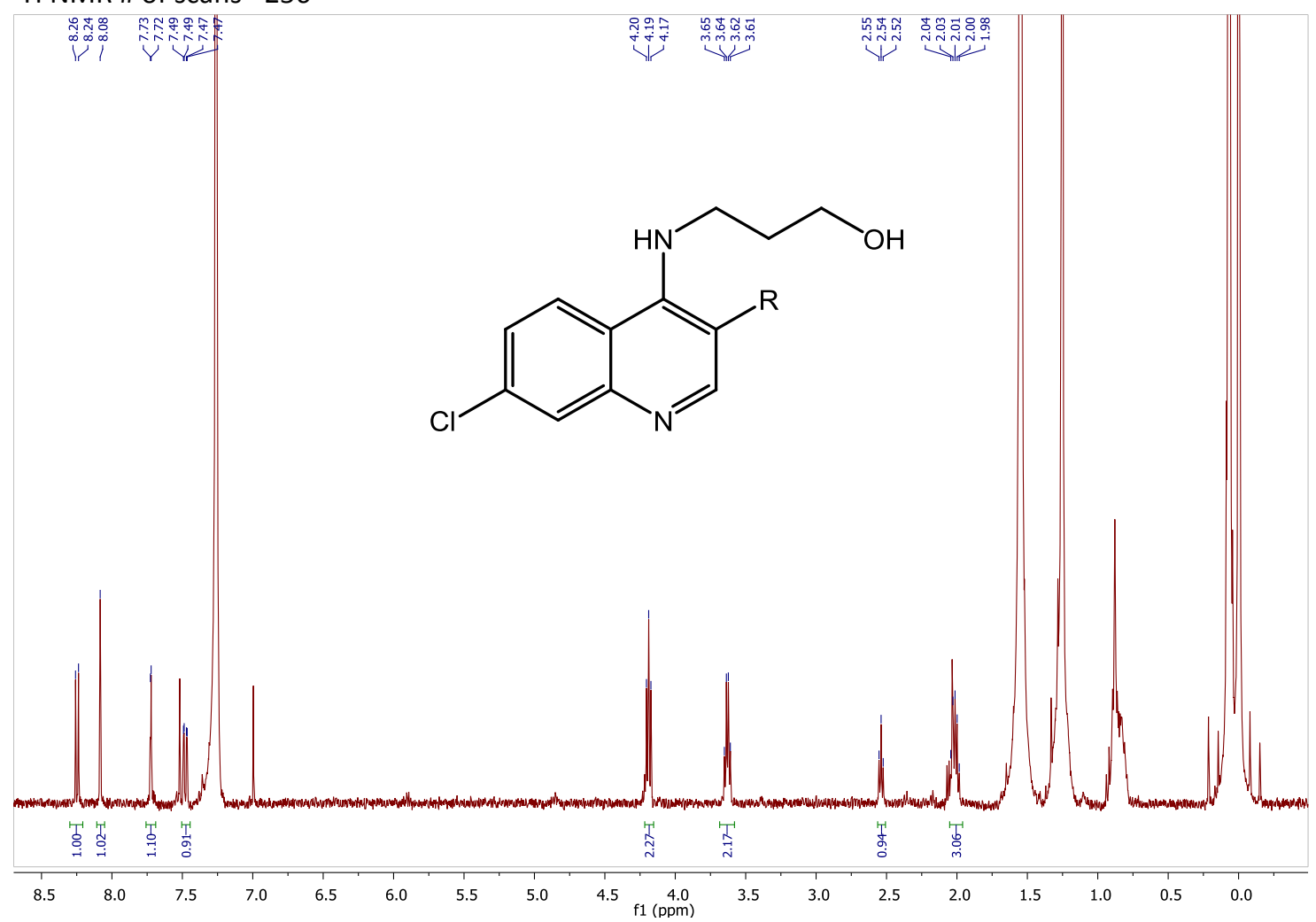



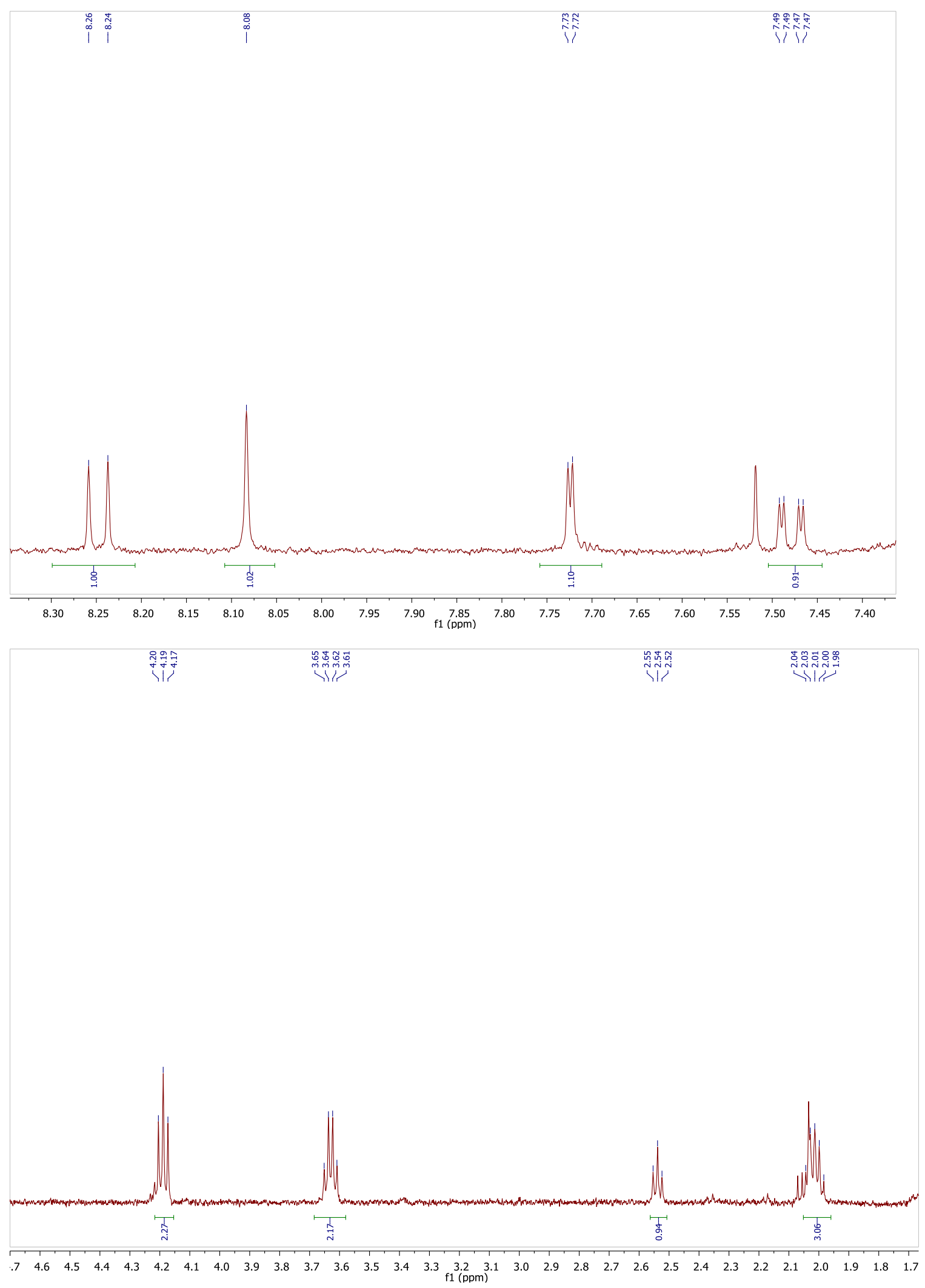


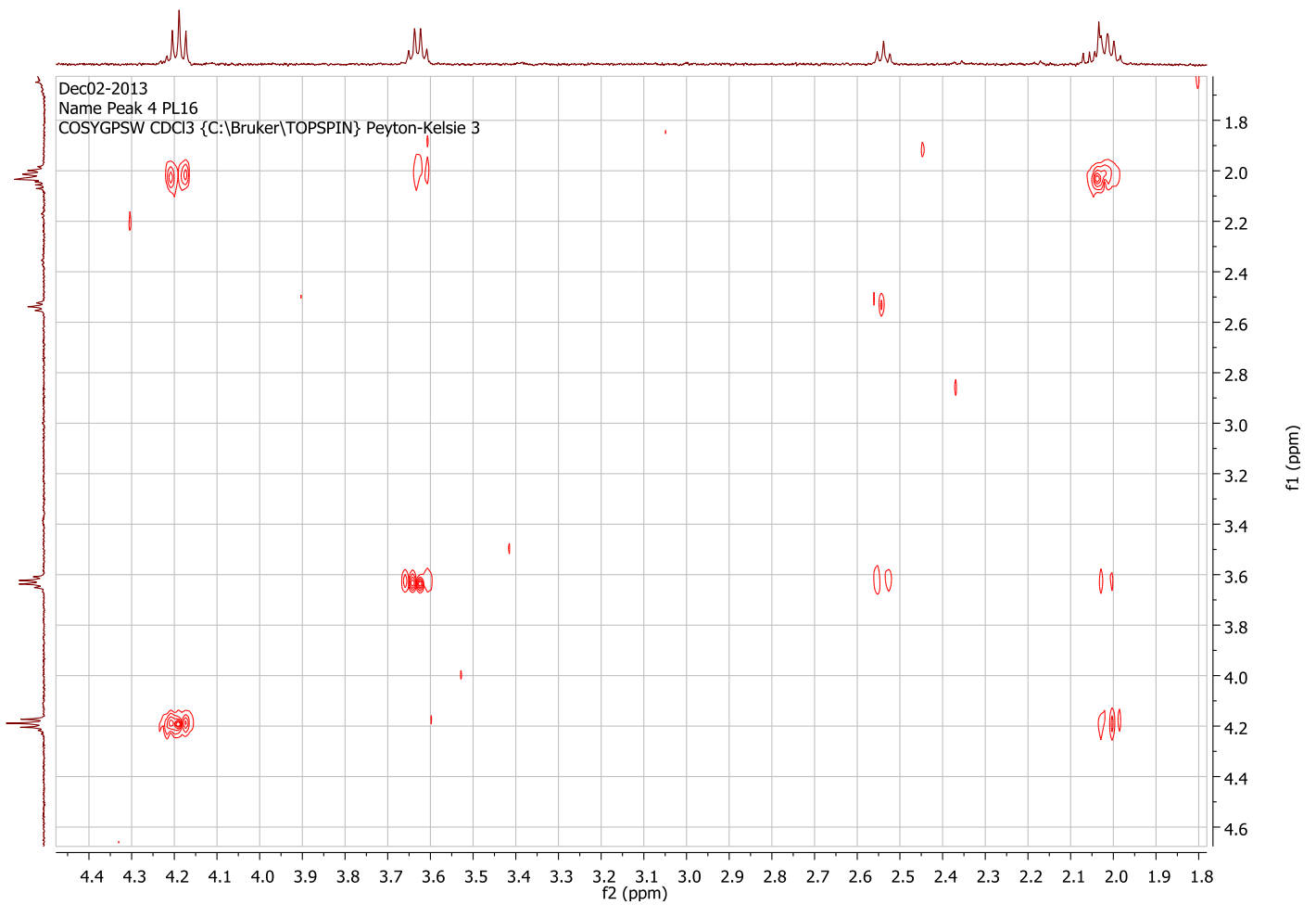

